\title{
Dyson's Nonintersecting Brownian Motions with a Few Outliers
}

\author{
MARK ADLER \\ Brandeis University \\ JONATHAN DELÉPINE \\ Université Catholique de Louvain \\ AND \\ PIERRE VAN MOERBEKE \\ Université Catholique de Louvain \& Brandeis University
}

\begin{abstract}
Consider $n$ non-intersecting Brownian particles on $\mathbb{R}$ (Dyson Brownian motions), all starting from the origin at time $t=0$, and forced to return to $x=0$ at time $t=1$. For large $n$, the average mean density of particles has its support, for each $0<t<1$, on the interval $\pm \sqrt{2 n t(1-t)}$. The Airy process $\mathscr{A}(\tau)$ is defined as the motion of these non-intersecting Brownian motions for large $n$, but viewed from the curve $\mathscr{C}: \quad y=\sqrt{2 n t(1-t)}$ with an appropriate space-time rescaling. Assume now a finite number $r$ of these particles are forced to a different target point, say $a=\rho_{0} \sqrt{n / 2}>0$. Does it affect the Brownian fluctuations along the curve $\mathscr{C}$ for large $n$ ? In this paper, we show that no new process appears as long as one considers points $(y, t) \in \mathscr{C}$, such that $0<t<\left(1+\rho_{0}^{2}\right)^{-1}$, which is the $t$-coordinate of the point of tangency of the tangent to the curve passing through $\left(\rho_{0} \sqrt{n / 2}, 1\right)$. At this point of tangency the fluctuations obey a new statistics, which we call the Airy process with $r$ outliers $\mathscr{A}^{(r)}(\tau)$ (in short: $r$-Airy process). The log of the probability that at time $\tau$ none of the particles in the cloud exceeds $x$ is given by the Fredholm determinant of a new kernel (extending the Airy kernel) and it satisfies a non-linear PDE in $x$ and $\tau$, from which the asymptotic behavior of the process can be deduced for $\tau \rightarrow-\infty$. This kernel is closely related to one found by Baik, Ben Arous and Péché in the context of multivariate statistics.
\end{abstract}

(c) 2000 Wiley Periodicals, Inc.

\section{Contents}

1. A constrained Brownian motion with a few outliers 10

2. The existence of the limit to the $r$-Airy kernel 15

3. An integrable deformation of Gaussian random ensemble with external source and 3-component KP

4. Virasoro constraints

(C) 2000 Wiley Periodicals, Inc. 
5. A PDE for the Gaussian ensemble with external source

6. A PDE for the transition probability of the $r$-Airy process

7. Remote past asymptotics

8. The $r$-Airy process, an interpolation between the Airy and Pearcey processes

9. Appendix

Dyson [16] made the important observation that putting dynamics into random matrix models leads to finitely many non-intersecting Brownian motions (on $\mathbb{R}$ ) for the eigenvalues. Applying scaling limits to the random matrix models, combined with Dyson's dynamics, then leads to interesting infinitely many diffusions for the eigenvalues. This paper studies a model, which stems from multivariate statistics and which interpolates between the Airy and Pearcey processes.

Consider $n$ non-intersecting Brownian particles on the real line $\mathbb{R}$,

$$
-\infty<x_{1}(t)<\ldots<x_{n}(t)<\infty,
$$

with (local) Brownian transition probability given by

$$
p(t ; x, y):=\frac{1}{\sqrt{\pi t}} e^{-\frac{(y-x)^{2}}{t}},
$$

all starting from the origin $x=0$ at time $t=0$, and forced to return to $x=0$ at time $t=1$. For very large $n$, the average mean density of particles has its support, for each $0<t<1$, on the interval $(-\sqrt{2 n t(1-t)}, \sqrt{2 n t(1-t)})$, as sketched in Figure 0.1 .

The Airy process $A(\tau)$ is defined as the motion of these non-intersecting Brownian motions for large $n$, but viewed from an observer on the (right hand) edgecurve

$$
\mathscr{C}:\{y=\sqrt{2 n t(1-t)}>0\}
$$

of the set of particles, with space stretched by the customary GUE-edge rescaling $n^{1 / 6}$ and time rescaled by the factor $n^{1 / 3}$ in tune with the Brownian motion spacetime rescaling; this is to say that in this new scale, slowed down microscopically, the left-most particles appear infinitely far and the time horizon $t=1$ lies in the very remote future. Thus, the Airy process describes the fluctuations of the Brownian particles near the edge-curve $\mathscr{C}$, looked at through a magnifying glass, as shown in Figure 0.1

The Airy process was introduced by Prähofer and Spohn [25] and further investigated in [20, 21, 27, 3]. Notice that in this work the Airy process is not viewed as the motion of the largest particle (point process), but as the motion of the cloud of particles, which will be described as a determinantal process. Giving a pathwise description of this motion remains an open problem. 


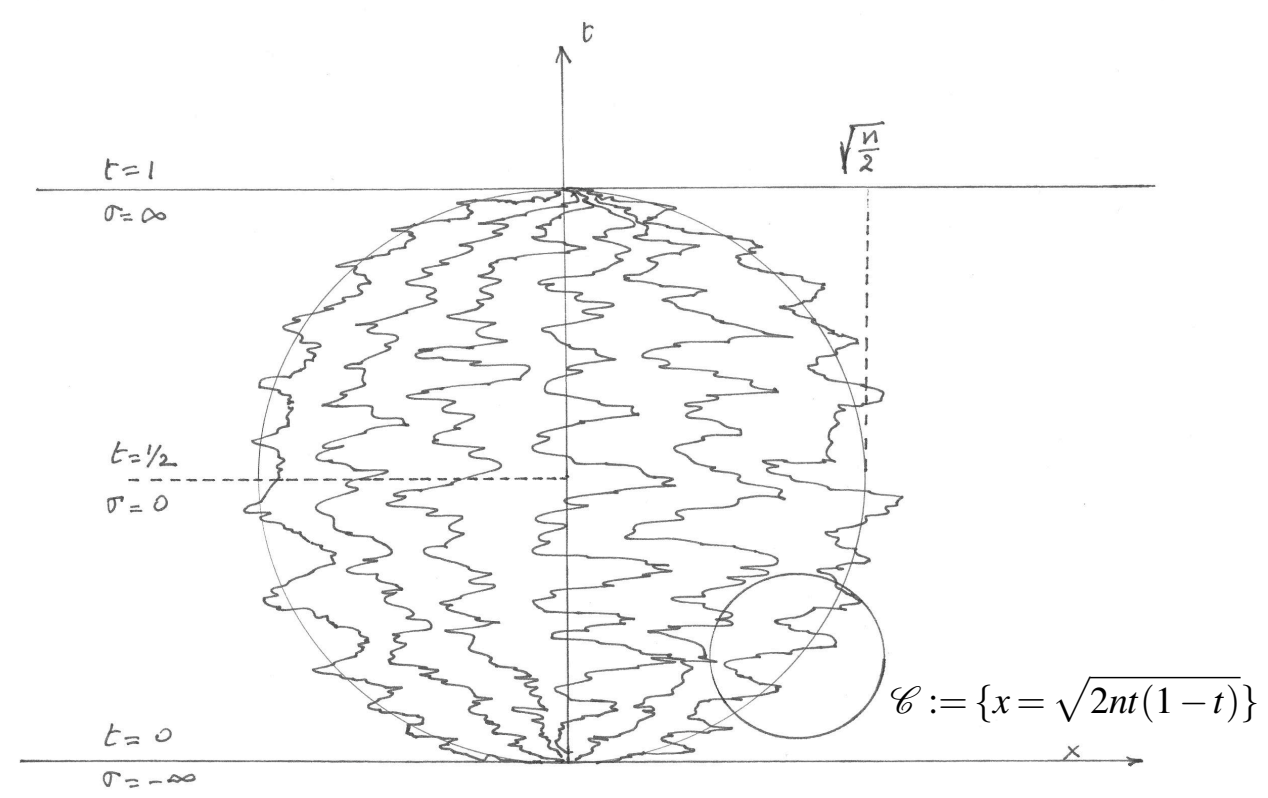

FIGURE 0.1. Airy process

Assume now that, among those $n$ paths, $0 \leq r \leq n$ are forced to reach a given final target $a \geq 0$, while the $(n-r)$ remaining particles return to the position $x=0$. Consider the probability that, at any given time $0<t<1$, all of the particles avoid a window $E \subset \mathbb{R}$, namely (the superscript in the probability $\mathbb{P}_{B r}^{0 a}$ here and later refers to the target points)

$$
\mathbb{P}_{B r}^{0 a}\left(\text { all } x_{j}(t) \in E^{c}\right):=\mathbb{P}\left(\begin{array}{l|l}
\text { all } x_{j}(t) \in E^{c} & \begin{array}{c}
\text { all } x_{j}(0)=0 \\
r \text { right paths end up at } a \text { at } t=1 \\
(n-r) \text { paths end up at } 0 \text { at } t=1
\end{array}
\end{array}\right) .
$$

Does the fact that a finite number $r$ of particles are forced to a different target point, in particular the target point $a=\rho_{0} \sqrt{n / 2}>0$ for some arbitrary parameter $\rho_{0}$, affect the Brownian fluctuations along the curve $\mathscr{C}$ for very large $n$ ? It is understood here that near the points of the curve (under consideration), one uses the same scaling as the Airy process. In this paper, we show that no new process appears as long as one considers points

$$
(y, t) \in \mathscr{C} \text {, such that } 0<t<\frac{1}{1+\rho_{0}^{2}} .
$$




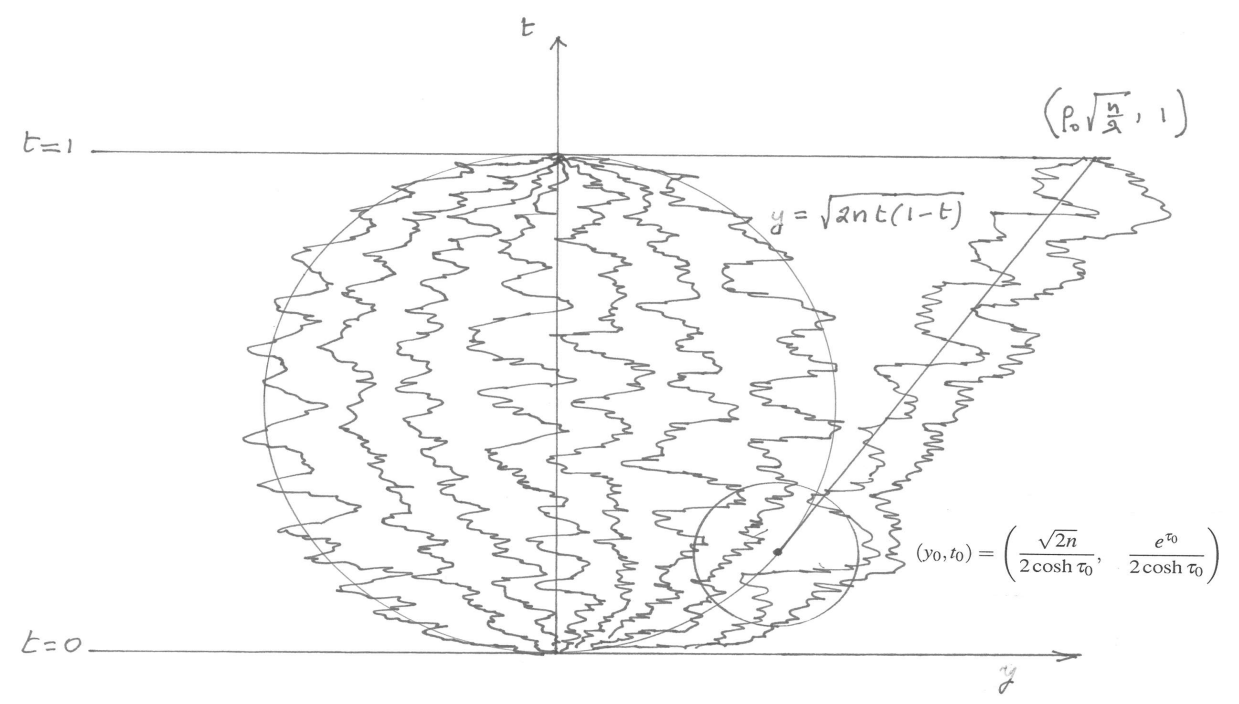

FIGURE 0.2 . The $r$-Airy process

Observe that $t=t_{0}=\left(1+\rho_{0}^{2}\right)^{-1}$ corresponds to the point of tangency $\left(y_{0}, t_{0}\right)$ of the tangent to the curve passing through the point $\left(\rho_{0} \sqrt{n / 2}, 1\right)$ of the $(t=1)$-axis; i.e.

$$
\left(y_{0}, t_{0}\right)=\left(\frac{\rho_{0} \sqrt{2 n}}{1+\rho_{0}^{2}}, \frac{1}{1+\rho_{0}^{2}}\right) \in \mathscr{C}
$$

At this point of tangency $\left(y_{0}, t_{0}\right)$ the fluctuations obey a new statistics, which we call the Airy process with $r$ outliers $\mathscr{A}^{(r)}(\tau)$ (in short: $r$-Airy process); see Figure 0.2 In particular, for the target point $a=\sqrt{n / 2}$, the $r$-Airy process occurs at the the maximum of the edge curve $\mathscr{C}$; i.e., $\rho_{0}=1$ and thus $t_{0}=1 / 2$. Notice, the $r$ Airy process is an extension of the Airy process; the $r$-Airy process coincides with the Airy process, when the target point $a$ coincides with 0 or, what is the same, when $r=0$. The Airy process is stationary, whereas the $r$-Airy process ceases to be stationary.

Given a target point $a=\rho_{0} \sqrt{n / 2}$, the point of tangency of the tangent to the curve $\mathscr{C}$, passing through $a$, can be written, in accordance with $(0.4)$,

$(0.5) \quad\left(y_{0}, t_{0}\right)=\left(\frac{\rho_{0} \sqrt{2 n}}{1+\rho_{0}^{2}}, \frac{1}{1+\rho_{0}^{2}}\right)=\left(y_{0}, t_{0}\right)=\left(\frac{\sqrt{2 n}}{2 \cosh \tau_{0}}, \quad \frac{e^{\tau_{0}}}{2 \cosh \tau_{0}}\right) \in \mathscr{C}$ 
upon introducing a new parameter $\tau_{0}$, whose significance as a new time parameter will be clear from section 1 (formula $(1.8)$ ), and which is defined by

$$
e^{-\tau_{0}}:=\rho_{0}=\sqrt{\frac{1-t_{0}}{t_{0}}} .
$$

Given a subset $E \subset \mathbb{R}$, the $r$-Airy process in the new time $\tau$ will be obtained by taking the following limit:

$$
\begin{aligned}
& \mathbb{P}\left(\mathscr{A}^{(r)}(\tau) \cap E=\emptyset\right) \\
& :=\lim _{n \rightarrow \infty} \mathbb{P}_{B r}^{\left(0, \rho_{0} \sqrt{n / 2}\right)}\left(\text { all } x_{i}\left(\frac{1}{1+e^{-2\left(\tau_{0}+\frac{\tau}{n^{1 / 3}}\right)}}\right) \in \frac{\sqrt{2 n}+\frac{E^{c}}{\sqrt{2} n^{1 / 6}}}{2 \cosh \left(\tau_{0}+\frac{\tau}{n^{1 / 3}}\right)}\right) .
\end{aligned}
$$

Notice that for $\tau=0$ and upon ignoring the set $E^{c}$, space and time on the right hand side of the formula above equal $\left(y_{0}, t_{0}\right)$ as in $(0.5)$. In section 2 , the limit above will be shown to exist, independently of the parameter $\rho_{0}$, at the same time establishing some universality.

In order to state Theorem 0.1 below, define the functions 1

$$
A_{r}^{ \pm}(u ; \tau):=\int_{C} e^{\frac{1}{3} i a^{3}+i a u}(\mp i a-\tau)^{ \pm r} \frac{d a}{2 \pi}
$$

and the standard Airy function $A(u):=A_{0}^{-}(u ; \tau)=A_{0}^{+}(u ; \tau)$, satisfying the differential equation $A^{\prime \prime}(x)=x A(x)$. Given these functions, the Airy and $r$-Airy kernels are defined by 0.9 below and will be used in Theorem 0.1 .

$$
\begin{aligned}
& K^{(0)}(u, v)=\int_{0}^{\infty} d w A(w+u) A(w+v)=\frac{A(u) A^{\prime}(v)-A^{\prime}(u) A(v)}{u-v} \\
& K_{\tau}^{(r)}(u, v)=\int_{0}^{\infty} d w A_{r}^{-}(w+u ; \tau) A_{r}^{+}(w+v ; \tau) .
\end{aligned}
$$

We now state:

Theorem 0.1. Consider non-intersecting Brownian motions as above with $r$ particles forced to a target point $\rho_{0} \sqrt{n / 2}>0$ at time $t=1$. For large $n$, the average mean density of particles as a function of $t$ has its support on a region bounded to the right by the curve $\mathscr{C}$ (defined just below 0.1$)$ ). The tangent line to $\mathscr{C}$, passing through $\left(\rho_{0} \sqrt{n / 2}, 1\right)$ has its point of tangency at $\left(y_{0}, t_{0}\right)$, given by $(0.4)$. Letting $n \rightarrow \infty$ and given an arbitrary point $(y, t) \in \mathscr{C}$ with $t \leq t_{0}$, a phase transition occurs at $t=t_{0}$, which is conveniently expressed in terms of the new parameters defined

\footnotetext{
${ }^{1} C$ is a contour running from $\infty e^{5 i \pi / 6}$ to $\infty e^{i \pi / 6}$, such that $-i \tau$ lies above the contour.
} 


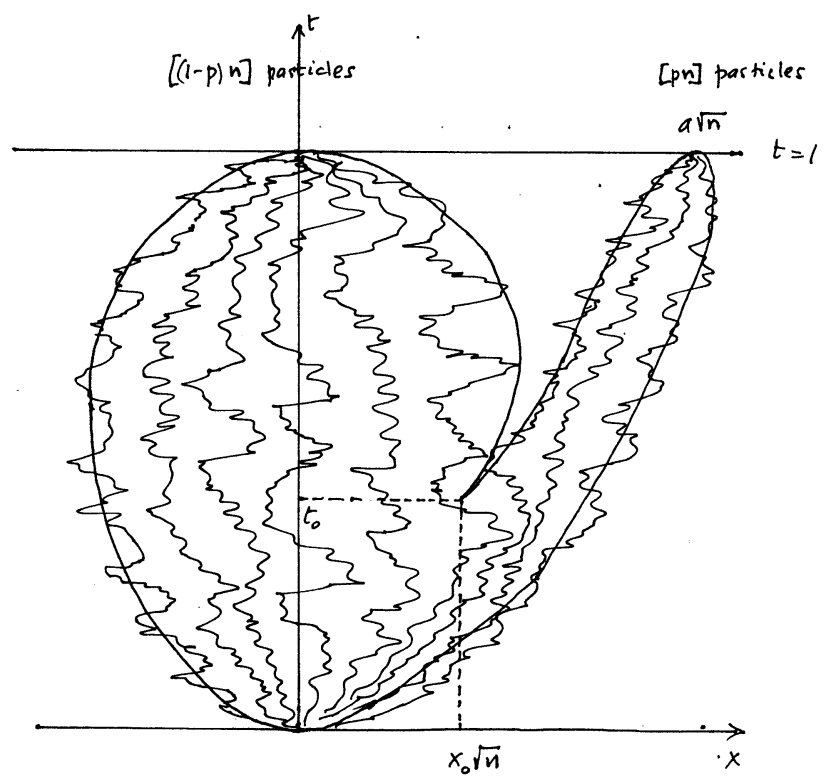

FIGURE 0.3. Pearcey process

by $y^{2} t=\left(1+e^{-2 \sigma}\right)^{-1}$ and $t_{0}=\left(1+e^{-2 \tau_{0}}\right)^{-1}$; one has the following limit:

$$
\begin{aligned}
& \lim _{n \rightarrow \infty} \mathbb{P}_{B r}^{\left(0, \rho_{0} \sqrt{n / 2}\right)}\left(\operatorname{all} x_{i}\left(\frac{1}{1+e^{-2\left(\sigma+\frac{\tau}{n^{1 / 3}}\right)}}\right) \in \frac{\sqrt{2 n}+\frac{E^{c}}{\sqrt{2} n^{1 / 6}}}{2 \cosh \left(\sigma+\frac{\tau}{n^{1 / 3}}\right)}\right) \\
& =\left\{\begin{array}{lll}
\mathbb{P}(\mathscr{A}(\tau) \cap E=\emptyset)=\operatorname{det}\left(I-K^{(0)}\right)_{E} & \text { for } \quad \begin{array}{l}
0 \leq t<t_{0} \\
\end{array} \\
& \left(\text { i.e., } 0 \leq \sigma<\tau_{0}\right), \\
\mathbb{P}\left(\mathscr{A}^{(r)}(\tau) \cap E=\emptyset\right)=\operatorname{det}\left(I-K_{\tau}^{(r)}\right)_{E} \quad \text { for } \quad t=t_{0} & \left(\text { i.e., } \sigma=\tau_{0}\right) .
\end{array}\right.
\end{aligned}
$$

These Fredholm determinants are genuine probability distributions for each value of $\tau$. Notice that for $0 \leq \sigma<\tau_{0}$, the limit above is independent of the time $\tau$ and the number of outliers $r$, unlike the case $\sigma=\tau_{0}$.

As is clear from Figure 0.2 and for $t>t_{0}$, the $r$ outliers separate from the bulk as a group; thus after an appropriate shift to take into account their displacement from the curve $\mathscr{C}$ and using a different scaling, they behave probabilistically like the $r$ eigenvalues of an $r \times r$ matrix in the GUE ensemble.

The $r$-Airy process can also be viewed as an interpolation between the Airy and Pearcey processes. The Pearcey process [28, 23, 4, 5] is defined as the limit of

\footnotetext{
${ }^{2}$ Remember from $\sqrt{0.6}$, one has $\rho_{0}=e^{-\tau_{0}}$.
} 
non-intersecting Brownian motions (for large $n$ ), all leaving from 0 at time $t=0$, with $(1-p) n$ paths forced to end up at 0 and $p n$ paths forced to end up at $a \sqrt{n / 2}$ at time $t=1$; see Figure 0.3. The Pearcey process then describes this Brownian cloud of particles (for $n \rightarrow \infty$ ) near the time $t$ of bifurcation, where the support of the average mean density goes from one interval into two intervals, with stretched space and time. Then the boundary of the support of the average mean density of particles in $(y, t)$-space has a cusp. In section 8 it will be shown sketchily how the location of the cusp, when the proportion $p$ of particles tends to 0 as $r / n$, tends to the precise place $\left(y_{0}, t_{0}\right)$ where the $r$-Airy process occurs.

Remark 0.2 . To simplify the notation, one often writes

$$
\mathbb{P}\left(\sup \mathscr{A}^{(r)}(\tau) \leq x\right):=\mathbb{P}\left(\mathscr{A}^{(r)}(\tau) \cap(x, \infty)=\emptyset\right) .
$$

Remark 0.3. The joint probabilities for the $r$-Airy processes for a finite number of times can be defined in a similar way and lead to a matrix Fredholm determinant, to be discussed in a later paper.

This phenomenon is closely related to statistical work by Baik-Ben ArousPéché [8], Baik [9] and Péché [29]. Indeed, consider a (complex) Gaussian population $\vec{y} \in \mathbb{C}^{N}$, with covariance matrix $\Sigma$. Given $M$ samples $\overrightarrow{y_{1}}, \ldots, \overrightarrow{y_{M}}$, the (centered) sample covariance matrix $S:=\frac{1}{M} X \bar{X}^{\top}$, where

$$
X=\left(\overrightarrow{y_{1}}-\frac{1}{M} \sum_{1}^{M} \overrightarrow{y_{i}}, \ldots, \overrightarrow{y_{M}}-\frac{1}{M} \sum_{1}^{M} \overrightarrow{y_{i}}\right),
$$

is a positive definite matrix and is an estimator of the true covariance matrix $\Sigma$. One may test the statistical hypothesis that $\Sigma=I$ or that $\Sigma$ has all eigenvalues $=1$, except for a few outliers. When all the eigenvalues of $\Sigma$ are $=1$, then the limit distribution of the largest eigenvalue of the sample covariance matrix $S$, for $N$ and $M$ tending to $\infty$ in the same way, is given by the Tracy-Widom distribution. Then Baik-BenArous-Péché [8] noticed that, this is still so, if the eigenvalues of $\Sigma$ contain some outliers, which are not too large. There is a critical point at which and beyond which the largest eigenvalue of the sample covariance matrix $S$ will be different from the Tracy-Widom distribution. At this point of phase transition Baik-BenArous-Péché [8] have found an Airy-type distribution in $x$, which is given by the Fredholm determinant

$$
\left.\operatorname{det}\left(I-K_{\tau}^{(r)}\right)_{(x, \infty)}\right|_{\tau=0},
$$

where $r$ denotes the number of eigenvalues of $\Sigma$ that are equal to $1+\gamma^{-1}$, while all the others are $=1 ; \gamma$ is such that $M / N=\gamma^{2}$ for $M$ and $N$ very large. This distribution was further generalized in [8] to the case where $\tau \neq 0$ in the kernel $K_{\tau}^{(r)}$. Baik proved in [9] that the Fredholm determinant of $K_{\tau}^{(r)}$ is a genuine probability distribution. In the statistical problem above, the covariance matrix $S$ is positive definite and therefore its eigenvalues satisfy Laguerre-type distributions; this idea 
was extended to GUE-type distributions by S. Péché [29]. In the present paper one finds that the shift $\tau$ appearing in the kernel $K_{\tau}^{(r)}$ is precisely the rescaled time of the non-intersecting Brownian motion model! The arguments will appear in sections 1 and 2 .

This paper also shows that the probability of the $r$-Airy process or, what is the same, the Fredholm determinant $\operatorname{det}\left(I-K_{\tau}^{(r)}\right)_{(x, \infty)}$ satisfies a non-linear PDE in $x$ and $\tau$, depending on the number $r$ of outliers, as established in section 6 .

Theorem 0.4. The logarithm of the probability

$$
Q(\tau, x):=\log \mathbb{P}\left(\sup \mathscr{A}^{(r)}(\tau) \leq x\right)=\log \operatorname{det}\left(I-K_{\tau}^{(r)}\right)_{(x, \infty)}
$$

satisfies the following non-linear PDE ${ }^{3}$, with both, the function $Q(\tau, x)$ and the $P D E$, being invarian 4 under the involution $(\tau, x, r) \rightarrow(-\tau, x,-r)$,

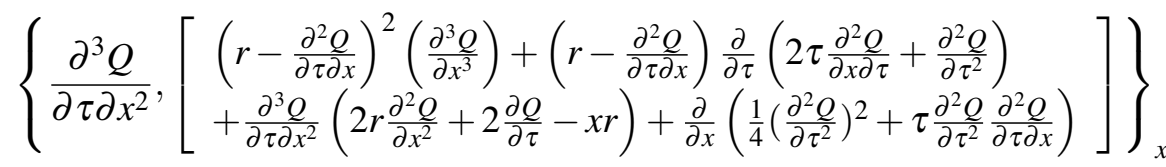

$$
\begin{aligned}
& -\frac{1}{2}\left(\frac{\partial^{3} Q}{\partial \tau \partial x^{2}}\right)^{2}\left(\frac{\partial^{3} Q}{\partial \tau^{3}}-4 \frac{\partial^{2} Q}{\partial \tau \partial x} \frac{\partial^{3} Q}{\partial x^{3}}\right)=0,
\end{aligned}
$$

with "initial condition", given by the log of the Tracy-Widom distribution,

$$
Q_{0}(x):=\lim _{\tau \rightarrow-\infty} Q(\tau, x):=\log \mathbb{P}(\sup \mathscr{A}(\tau) \leq x)=-\int_{x}^{\infty}(\alpha-x) g^{2}(\alpha) d \alpha
$$

where $g(\alpha)$ is the Hastings-MacLeod solution of Painlevé II,

$$
g^{\prime \prime}=\alpha g+2 g^{3}, \text { with } g(\alpha) \cong \frac{e^{-\frac{2}{3} \alpha^{\frac{3}{2}}}}{2 \sqrt{\pi} \alpha^{1 / 4}} \text { for } \alpha \nearrow_{\infty} .
$$

Remark 0.5. Obviously, the PDE 0.12 has the following structure

$$
\frac{\partial}{\partial x}\left(\frac{\cdots}{\frac{\partial^{3} Q}{\partial \tau \partial x^{2}}}\right)=\frac{\partial^{3} Q}{\partial \tau^{3}}-4 \frac{\partial^{2} Q}{\partial \tau \partial x} \frac{\partial^{3} Q}{\partial x^{3}}
$$

Remark 0.6. The following simple recipe gives the PDE for $\mathbb{P}\left(\mathscr{A}^{(r)}(\tau) \cap E=\emptyset\right)$ for a general set $E=\cup_{1}^{\ell}\left[x_{2 i-1}, x_{2 i}\right]$, replacing the PDE 0.12 for $E=(x, \infty)$; indeed, perform the replacements

$$
\frac{\partial}{\partial x} \mapsto \sum_{i} \frac{\partial}{\partial x_{i}} \text { and } x\left(\frac{\partial}{\partial x}\right)^{k} \mapsto\left(\sum_{i} x_{i} \frac{\partial}{\partial x_{i}}\right)\left(\sum_{i} \frac{\partial}{\partial x_{i}}\right)^{k-1}
$$

\footnotetext{
${ }^{3}$ The Wronskian $\{f, g\}_{x}$ with regard to the variable $x$ is defined as $f^{\prime} g-f g^{\prime}$.

${ }^{4}$ The invariance under the involution is obvious for the equation; for the function $Q(\tau, x)$, see Lemma 7.5.
} 
with the understanding that differentiation must always be pulled to the right.

Although the average mean density of the particles is insensitive to the presence of outliers, the presence of the $r$ particles forced to reach the target $a>0$ at $t=1$ is already felt, when $t \rightarrow 0$ in the $t$-scale; that is when $\tau \rightarrow-\infty$ in the $\tau$ scale. The net effect is that it pulls the edge of the cloud of particles in the average towards the right to first order like $|r / \tau|$; i.e., the more so when $r$ gets large; this edge then behaves like the Airy process shifted in space, up to and including order $1 / \tau^{4}$.The PDE in Theorem 0.4 is a convenient instrument to extract the remote past asymptotics, as shown in Section 7 and stated in the theorem below.

Theorem 0.7. The PDE with the initial condition $Q_{0}(x)$ as in $(0.13)$ admits the asymptotic solution, for $\tau \rightarrow-\infty$, of the form

$$
Q(\tau, x)=\sum_{0}^{\infty} \frac{Q_{i}(x)}{\tau^{i}}=Q_{0}\left(\left(x+\frac{r}{\tau}\right)\left(1+\frac{r}{3 \tau^{3}}\right)+\frac{r^{2}}{4 \tau^{4}}\right)+\frac{r}{5 \tau^{5}} \mathscr{F}_{5}+O\left(\frac{1}{\tau^{6}}\right) .
$$

For the probability itself, one has 5

$$
\begin{aligned}
& \mathbb{P}\left(\sup \mathscr{A}^{(r)}(\tau) \leq x\right) \\
& =\mathbb{P}\left(\sup \mathscr{A}(\tau) \leq\left(x+\frac{r}{\tau}\right)\left(1+\frac{r}{3 \tau^{3}}\right)+\frac{r^{2}}{4 \tau^{4}}\right)\left(1+\frac{r}{5 \tau^{5}} \mathscr{F}_{5}+O\left(\frac{1}{\tau^{6}}\right)\right) .
\end{aligned}
$$

For $\tau \rightarrow-\infty$, the mean and variance of the right edge of the process behave as

$$
\begin{aligned}
\mathbb{E}\left(\sup \mathscr{A}^{(r)}(\tau)\right) & =\mathbb{E}\left(\sup \mathscr{A}^{(0)}(\tau)\right)\left(1-\frac{r}{3 \tau^{3}}\right)-\frac{r}{\tau}-\frac{r^{2}}{4 \tau^{4}}+O\left(\frac{1}{\tau^{5}}\right) \\
\operatorname{var}\left(\sup \mathscr{A}^{(r)}(\tau)\right) & =\operatorname{var}\left(\sup \mathscr{A}^{(0)}(\tau)\right)\left(1-\frac{2 r}{3 \tau^{3}}\right)+O\left(\frac{1}{\tau^{5}}\right) .
\end{aligned}
$$

The probability $(0.2)$ is related, via a change of variables, to a Gaussian matrix model with external potential,

$$
\mathbb{P}_{n}(\alpha, \tilde{E})=\frac{1}{Z_{n}} \int_{\mathscr{H}_{n}(\tilde{E})} d M e^{-\frac{1}{2} \operatorname{Tr}\left(M^{2}-2 A M\right)},
$$

where $\mathscr{H}_{n}(\tilde{E})$ denotes the Hermitian matrices with eigenvalues in $\tilde{E} \subset \mathbb{R}$ and with a diagonal matrix

$$
A:=\left(\begin{array}{cccccc}
\alpha & & & & & \\
& \ddots & & & & 0 \\
& & \alpha & & & \\
& & & 0 & & \\
& \mathbf{O} & & & \ddots & \\
& & & & & 0
\end{array}\right) .
$$

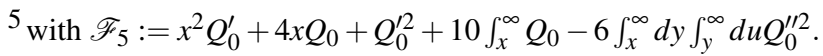


The relationship between the Karlin-McGregor non-intersecting Brownian motions and matrix models has been developped by Johansson [19] and, for the Gaussian matrix model with external potential, by Aptekarev-Bleher-Kuijlaars [7]. The latter model has come up in many other situations; among others, see [24, 11, 12, 13, 32, 33, 20, 10, 28, 4]. Following the method of Adler-van Moerbeke [4], using the multi-component KP hierarchy (section 3) and the Virasoro constraints (section 4), it is shown in section 5 that this matrix model satisfies a non-linear PDE in $\alpha$ and the boundary points of $\tilde{E}$. The PDE of Theorem 0.4 then follows from making an asymptotic analysis on that PDE. The asymptotic behavior of the $r$-Airy process for $\tau \rightarrow-\infty$ follows from solving the partial differential equation near $\tau=-\infty$ for the Airy-kernel initial condition; this process introduces some free constants, which then can be determined by the asymptotic properties of the $r$-Airy kernel for $x \rightarrow \infty$. It is an interesting question whether these results can be deduced via the Riemann-Hilbert methods, in the style of [15].

\section{A constrained Brownian motion with a few outliers}

The purpose of this section is to motivate the scaling limit (0.7) leading to the definition of the $r$-Airy process. The Airy process was originally defined as an edge scaling limit of Dyson's non-intersecting Brownian motions, in the same way that the Tracy-Widom distribution was obtained as an edge scaling limit of GUE. The non-intersecting Brownian motions $(0.2)$ with target point $a=0$ can be transformed to the Dyson Brownian motion, as will be explained in this section. This transformation will be used in the case of a $a \neq 0$ target. In the course of doing this, one must consider a Gaussian matrix model with external potential.

\subsection{Dyson Brownian motion}

The Dyson process ([16]) describes the motion of the eigenvalues $\lambda_{i}\left(t^{\prime}\right)$ of an $n \times n$ Hermitian random matrix $B\left(t^{\prime}\right)$ whose real and imaginary entries perform independent Ornstein-Uhlenbeck processes, given that the initial distribution is given by invariant measure for the process, namely

$$
Z^{-1} \int_{\mathscr{H}_{n}\left(E^{\prime}\right)} d B e^{-\operatorname{Tr} B^{2}}
$$

Then the process is stationary and its probability distribution at any time is given by

$$
\mathbb{P}_{D y}\left(\text { all } \lambda_{i}\left(t^{\prime}\right) \in E^{\prime}\right)=Z^{-1} \int_{\mathscr{H}_{n}\left(E^{\prime}\right)} d B e^{-\operatorname{Tr} B^{2}}=Z^{-1} \int_{\mathscr{H}_{n}\left(\sqrt{2} E^{\prime}\right)} d M e^{-\frac{1}{2} \operatorname{Tr} M^{2}}
$$


The probability for two times $0<t_{1}^{\prime}<t_{2}^{\prime}$ is then given by ( $\operatorname{set} c^{\prime}=e^{-\left(t_{2}^{\prime}-t_{1}^{\prime}\right)}$ )

$$
\begin{aligned}
& \mathbb{P}_{D y}\left(\text { all } \lambda_{i}\left(t_{1}^{\prime}\right) \in E_{1}^{\prime}, \text { all } \lambda_{i}\left(t_{2}^{\prime}\right) \in E_{2}^{\prime}\right) \\
& =P\left(\text { all }\left(B\left(t_{1}^{\prime}\right) \text {-eigenvalues }\right) \in E_{1}^{\prime}, \text { all }\left(B\left(t_{2}^{\prime}\right) \text {-eigenvalues }\right) \in E_{2}^{\prime}\right) \\
& =\iint_{\substack{\text { all } B_{1} \text {-eigenvalues } \in E_{1}^{\prime} \\
\text { all } B_{2} \text {-eigenvalues } \in E_{2}^{\prime}}} Z^{-1} \frac{d B_{1} d B_{2}}{\left(1-c^{\prime 2}\right)^{n^{2} / 2}} e^{-\frac{1}{1-c^{\prime 2}} \operatorname{Tr}\left(B_{1}^{2}+B_{2}^{2}-2 c^{\prime} B_{1} B_{2}\right)} \\
& =\iint_{M_{1} \in \mathscr{H}_{n}\left(\frac{\sqrt{2} E_{1}^{\prime}}{\left.\sqrt{1-c^{2}}\right)}\right.} Z^{\prime-1} d M_{1} d M_{2} e^{-\frac{1}{2} \operatorname{Tr}\left(M_{1}^{2}+M_{2}^{2}-2 c^{\prime} M_{1} M_{2}\right)} \\
& M_{2} \in \mathscr{H}_{n}\left(\frac{\sqrt{2} E_{2}^{\prime}}{\sqrt{1-c^{\prime 2}}}\right)
\end{aligned}
$$

\subsection{Constrained Brownian motion with target $a>0$}

As in the introduction, consider the $n$ non-intersecting Brownian particles on $\mathbb{R}$, all starting from the origin at time $t=0$, where among those paths, $1 \leq r \leq n$ are forced to end up at the target $a>0$, while the $(n-r)$ other paths return to the position $x=0$ at time $t=1$. Remember $\mathbb{P}_{B r}^{0 a}$ denotes the transition probability. Then the probability that all the particles belong to some window $E \subset \mathbb{R}$ at a given time $0<t<1$, can be expressed in terms of a Gaussian matrix model with an external potential, using the Karlin-McGregor formula [22] for non-intersecting Brownian motions (see [17, 19, 7, 4]), itself involving the Brownian transition probability (0.1), namely:

$$
\begin{aligned}
& \mathbb{P}_{B r}^{0 a}\left(\operatorname{all} x_{j}(t) \in E\right):=\mathbb{P}\left(\begin{array}{l|l}
\text { all } x_{j}(t) \in E & \begin{array}{c}
\text { all } x_{j}(0)=0 \\
r \text { right paths end up at } a \text { at } t=1 \\
(n-r) \text { paths end up at } 0 \text { at } t=1
\end{array}
\end{array}\right) \\
& =\quad \lim _{\text {all } \gamma_{i} \rightarrow 0} \quad \int_{E^{n}} \frac{1}{Z_{n}(\gamma, \delta)} \prod_{1}^{n} d x_{i} \\
& \delta_{1}, \ldots, \delta_{n-r} \rightarrow 0 \\
& \delta_{n-r+1}, \ldots, \delta_{n} \rightarrow a \\
& \operatorname{det}\left(p\left(t ; \gamma_{i}, x_{j}\right)\right)_{1 \leq i, j \leq n} \operatorname{det}\left(p\left(1-t ; x_{i^{\prime}}, \delta_{j^{\prime}}\right)\right)_{1 \leq i^{\prime}, j^{\prime} \leq n}, \\
& =\mathbb{P}_{n}\left(a \sqrt{\frac{2 t}{1-t}} ; E \sqrt{\frac{2}{t(1-t)}}\right)
\end{aligned}
$$


where 6

$$
\begin{aligned}
\mathbb{P}_{n}(\alpha, \tilde{E}) & =\frac{1}{Z_{n}} \int_{\mathscr{H}_{n}(\tilde{E})} d M e^{-\frac{1}{2} \operatorname{Tr}\left(M^{2}-2 A M\right)} \\
& =\frac{1}{Z_{n}^{\prime}} \int_{\tilde{E}^{n}} \Delta_{n}(x, y)\left(\Delta_{r}(x) \prod_{i=1}^{r} e^{-\frac{x_{i}^{2}}{2}+\alpha x_{i}} d x_{i}\right)\left(\Delta_{n-r}(y) \prod_{i=1}^{n-r} e^{-\frac{y_{i}^{2}}{2}} d y_{i}\right),
\end{aligned}
$$

with $A$ as in 0.16 . In short, the conditioned Brownian motion is related to a Gaussian matrix model with an external potential $A$ as follows:

$$
\mathbb{P}_{B r}^{0 a}\left(\operatorname{all} x_{j}(t) \in E\right)=\mathbb{P}_{n}(\alpha, \tilde{E}) \text { with } \tilde{E}=E \sqrt{\frac{2}{t(1-t)}}, \alpha=a \sqrt{\frac{2 t}{1-t}} .
$$

The joint probability for the constrained Brownian motion at two times is related to a chain of two Gaussian matrix models (see [5]) with an external potential $A$, which again by Karlin-McGregor reads as follows:

$$
\mathbb{P}_{B r}^{0 a}\left(\text { all } x_{i}\left(t_{1}\right) \in E_{1}, \text { all } x_{i}\left(t_{2}\right) \in E_{2}\right)=\mathbb{P}_{n}\left(\alpha ; c^{\prime} ; \tilde{E}_{1}, \tilde{E}_{2}\right),
$$

where ( $A$ is the same diagonal matrix as in 0.16 )

$$
\mathbb{P}_{n}\left(\alpha ; c^{\prime} ; \tilde{E}_{1}, \tilde{E}_{2}\right)=\frac{1}{Z_{n}} \int_{\mathscr{H}_{n}\left(\tilde{E}_{1}\right) \times \mathscr{H}_{n}\left(\tilde{E}_{2}\right)} e^{-\frac{1}{2} \operatorname{Tr}\left(M_{1}^{2}+M_{2}^{2}-2 c^{\prime} M_{1} M_{2}-2 A M_{2}\right)} d M_{1} d M_{2},
$$

with

$$
\begin{gathered}
\tilde{E}_{1}=E_{1} \sqrt{\frac{2 t_{2}}{\left(t_{2}-t_{1}\right) t_{1}}}, \tilde{E}_{2}=E_{2} \sqrt{\frac{2\left(1-t_{1}\right)}{\left(1-t_{2}\right)\left(t_{2}-t_{1}\right)}} \\
c^{\prime}=\sqrt{\frac{\left(1-t_{2}\right) t_{1}}{\left(1-t_{1}\right) t_{2}}}=\frac{\sqrt{\frac{t_{1}}{1-t_{1}}}}{\sqrt{\frac{t_{2}}{1-t_{2}}}}, \alpha=a \sqrt{\frac{2\left(t_{2}-t_{1}\right)}{\left(1-t_{2}\right)\left(1-t_{1}\right)}}
\end{gathered}
$$

\subsection{Comparing the Dyson and constrained Brownian motions with tar- get $a=0$}

From the identities $(1.1)$ and $(1.3)$, one deduces the following set of identities for one time $t^{\prime}$ (in the time $t^{\prime}$ of the Dyson process), setting the point $a=0$,

$$
\mathbb{P}_{D y}\left(\text { all } \lambda_{j}\left(t^{\prime}\right) \in E^{\prime}\right)=\left.\mathbb{P}_{n}(\alpha, \tilde{E})\right|_{\alpha=0}=\left.\mathbb{P}_{B r}^{0 a}\left(\text { all } x_{j}(t) \in E\right)\right|_{a=0}
$$

where

$$
\sqrt{2} E^{\prime}=\tilde{E}=E \sqrt{\frac{2}{t(1-t)}}
$$

\footnotetext{
${ }^{6} \Delta_{r}(x)$ denotes the Vandermonde determinant, with regard to the $r$ variables $x=\left(x_{1}, \ldots, x_{r}\right)$ and $\Delta_{n}(x, y)$ denotes the Vandermonde determinant, with regard to the $n$ variables $x=\left(x_{1}, \ldots, x_{r}\right)$ and $y=\left(y_{1}, \ldots, y_{n-r}\right)$.
} 
Similarly, for two times $t_{1}^{\prime}$ and $t_{2}^{\prime}$, one deduces from the identities 1.2$]$ and 1.4 ,

$$
\begin{aligned}
\mathbb{P}_{D y}\left(\text { all } \lambda_{i}\left(t_{1}^{\prime}\right) \in E_{1}^{\prime} \text {, all } \lambda_{i}\left(t_{2}^{\prime}\right) \in E_{2}^{\prime}\right) & =\left.\mathbb{P}_{n}\left(\alpha ; c^{\prime} ; \tilde{E}_{1}, \tilde{E}_{2}\right)\right|_{\alpha=0} \\
& =\left.\mathbb{P}_{B r}^{0 a}\left(\text { all } x_{i}\left(t_{1}\right) \in E_{1} \text {, all } x_{i}\left(t_{2}\right) \in E_{2}\right)\right|_{a=0},
\end{aligned}
$$

where, as follows from (1.2) and (1.5), one deduces

$$
\begin{aligned}
& \frac{\sqrt{2} E_{1}^{\prime}}{\sqrt{\left(1-c^{\prime 2}\right)}}=\tilde{E}_{1}=E_{1} \sqrt{\frac{2 t_{2}}{\left(t_{2}-t_{1}\right) t_{1}}} \\
& \frac{\sqrt{2} E_{2}^{\prime}}{\sqrt{\left(1-c^{\prime 2}\right)}}=\tilde{E}_{2}=E_{2} \sqrt{\frac{2\left(1-t_{1}\right)}{\left(1-t_{2}\right)\left(t_{2}-t_{1}\right)}}
\end{aligned}
$$

with

$$
c^{\prime}=\frac{e^{t_{1}^{\prime}}}{e^{t_{2}^{\prime}}}=\frac{\sqrt{\frac{t_{1}}{1-t_{1}}}}{\sqrt{\frac{t_{2}}{1-t_{2}}}} .
$$

Thus the processes are related by a clock change

$$
e^{t_{i}^{\prime}}=\sqrt{\frac{t_{i}}{1-t_{i}}} \Longleftrightarrow t_{i}=\frac{1}{1+e^{-2 t_{i}^{\prime}}} \Longleftrightarrow \frac{1}{\sqrt{t_{i}\left(1-t_{i}\right)}}=2 \cosh t_{i}^{\prime},
$$

and thus $1-c^{\prime 2}=\frac{t_{2}-t_{1}}{t_{2}\left(1-t_{1}\right)}$. Comparing extremities in 1.77 , one finds

$$
\begin{gathered}
E_{1}^{\prime}=E_{1} \sqrt{\frac{2 t_{2}}{\left(t_{2}-t_{1}\right) t_{1}}} \sqrt{\left(1-c^{\prime 2}\right) / 2}=E_{1} \frac{1}{\sqrt{t_{1}\left(1-t_{1}\right)}} \\
E_{2}^{\prime}=E_{2} \sqrt{\frac{2\left(1-t_{1}\right)}{\left(1-t_{2}\right)\left(t_{2}-t_{1}\right)}} \sqrt{\left(1-c^{\prime 2}\right) / 2}=E_{2} \frac{1}{\sqrt{t_{2}\left(1-t_{2}\right)}} .
\end{gathered}
$$

This fact, combined with (1.8), yields

$$
E_{i}=E_{i}^{\prime} \sqrt{t_{i}\left(1-t_{i}\right)}=\frac{E_{i}^{\prime}}{2 \cosh t_{i}^{\prime}} .
$$

So, summarizing (1.8) and (1.9), one has the relation between the parameters of the Dyson process and constrained Brownian motions,

$$
e^{t_{i}^{\prime}}=\sqrt{\frac{t_{i}}{1-t_{i}}}, E_{i} \sqrt{\frac{2}{t_{i}\left(1-t_{i}\right)}}=\sqrt{2} E_{i}^{\prime} .
$$

Theorem 1.1. (Tracy-Widom [26], Adler-van Moerbeke [3]) Taking a limit on the Dyson process, in an appropriate time and space scale, one finds the Airy process, which is stationary:

$$
\lim _{n \rightarrow \infty} \mathbb{P}_{D y}\left(\text { all } \lambda_{i}\left(\frac{\tau}{n^{1 / 3}}\right) \in\left(-\infty, \sqrt{2 n}+\frac{x}{\sqrt{2} n^{1 / 6}}\right)\right)=\mathbb{P}(\mathscr{A}(\tau) \leq x)=F(x),
$$


$F(x)$ being the Tracy-Widom distribution. Similarly the limit of the joint probability for the Dyson process yields the joint probability for the Airy process:

$$
\lim _{n \rightarrow \infty} \mathbb{P}_{D y}\left(\begin{array}{c}
\text { all } \lambda_{i}\left(\frac{\tau_{1}}{n^{1 / 3}}\right) \in\left(-\infty, \sqrt{2 n}+\frac{x_{1}}{\sqrt{2} n^{1 / 6}}\right) \\
\text { all } \lambda_{i}\left(\frac{\tau_{2}}{n^{1 / 3}}\right) \in\left(-\infty, \sqrt{2 n}+\frac{x_{2}}{\sqrt{2} n^{1 / 6}}\right)
\end{array}\right)=\mathbb{P}\left(\begin{array}{c}
\mathscr{A}\left(\tau_{1}\right) \leq x_{1}, \\
\mathscr{A}\left(\tau_{2}\right) \leq x_{2}
\end{array}\right) .
$$

Its logarithm (setting $s=\left(\tau_{2}-\tau_{1}\right) / 2$ )

$$
H(s ; x, y):=\log \mathbb{P}\left(\mathscr{A}\left(\tau_{1}\right) \leq x+y, \mathscr{A}\left(\tau_{2}\right) \leq x-y\right),
$$

satisfies the Airy PDE

$$
2 s \frac{\partial^{3} H}{\partial s \partial x \partial y}=\left(2 s^{2} \frac{\partial}{\partial y}-y \frac{\partial}{\partial x}\right)\left(\frac{\partial^{2} H}{\partial y^{2}}-\frac{\partial^{2} H}{\partial x^{2}}\right)+\left\{\frac{\partial^{2} H}{\partial x \partial y}, \frac{\partial^{2} H}{\partial x^{2}}\right\}_{x} .
$$

Corollary 1.2. Taking an appropriate scaling limit on the constrained Brownian motion, we have

$$
\left.\lim _{n \rightarrow \infty} \mathbb{P}_{B r}^{0 a}\left(\text { all } x_{i}\left(\frac{1}{1+e^{-2 \tau / n^{1 / 3}}}\right) \in \frac{\left(-\infty, \sqrt{2 n}+\frac{x}{\sqrt{2} n^{1 / 6}}\right)}{2 \cosh \left(\tau / n^{1 / 3}\right)}\right)\right|_{a=0}=\mathbb{P}(\mathscr{A}(\tau) \leq x)
$$

and similarly for two times.

Proof. This statement follows immediately from Theorem 1.1 and the correspondence (1.6), upon using the clock change (1.8) and the space change (1.9), with the appropriate time and space scalings of Theorem 1.1 .

Remembering the definition

$$
\mathbb{P}_{n}(\alpha, \tilde{E})=\frac{1}{Z_{n}} \int_{\mathscr{H}_{n}(\tilde{E})} d M e^{-\frac{1}{2} \operatorname{Tr}\left(M^{2}-2 A M\right)},
$$

for the diagonal matrix $A$ as in 0.16 , we now state a Theorem of Péché, which is closely related to the multivariate statistical problem mentioned in the introduction; see [8].

Theorem 1.3. (Péché [29])

$$
\lim _{n \rightarrow \infty} \mathbb{P}_{n}\left(\rho \sqrt{n},\left(-\infty, 2 \sqrt{n}+\frac{x}{n^{1 / 6}}\right)\right)=\left\{\begin{array}{l}
F(x) \text { for } \rho<1 \\
F^{(r)}(x) \text { for } \rho=1
\end{array}\right.
$$

where

$$
F^{(r)}(x)=\left.\operatorname{det}\left(I-K_{\tau}^{(r)} \chi_{(x, \infty)}(y)\right)\right|_{\tau=0}
$$

is the Fredholm determinant of the kernel (see (0.8))

$$
K_{\tau}^{(r)}(x, y)=\int_{0}^{\infty} d u A_{r}^{-}(x+u ; \tau) A_{r}^{+}(y+u ; \tau) .
$$

For $r=0$, (1.13) yields the Tracy-Widom distribution $F(x)$. 
The Airy process will now be deformed in a way which is compatible with Péché's Theorem, especially concerning the target point $a$,

$$
\mathbb{P}_{B r}^{(0 a)}\left(\operatorname{all} x_{i}\left(\frac{1}{1+e^{-2 \tau / n^{1 / 3}}}\right) \in \frac{\left(-\infty, \sqrt{2 n}+\frac{x}{\sqrt{2} n^{1 / 6}}\right)}{2 \cosh \left(\tau / n^{1 / 3}\right)}\right) .
$$

Since, from (1.3),

$$
\mathbb{P}_{B r}^{0 a}\left(\operatorname{all} x_{j}(t) \in E\right)=\mathbb{P}_{n}\left(a \sqrt{\frac{2 t}{1-t}} ; E \sqrt{\frac{2}{t(1-t)}}\right)
$$

holds, we have

$$
\begin{aligned}
\mathbb{P}_{B r}^{(0 a)}\left(\operatorname{all} x_{i}\left(\frac{1}{1+e^{-2 \tau / n^{1 / 3}}}\right)\right. & \left.\in \frac{\left(-\infty, \sqrt{2 n}+\frac{x}{\sqrt{2} n^{1 / 6}}\right)}{2 \cosh \left(\tau / n^{1 / 3}\right)}\right) \\
& =\mathbb{P}_{n}\left(a \sqrt{2} e^{\tau / n^{1 / 3}} ;\left(-\infty, 2 \sqrt{n}+\frac{x}{n^{1 / 6}}\right)\right) .
\end{aligned}
$$

Comparing this formula at time $t=1 / 2$, or what is the same at $\tau=0$, with formula (1.12) suggests the choice

$$
a=\rho \sqrt{\frac{n}{2}} .
$$

We thus define the Airy process with $r$ outliers (in short: $r$-Airy process) $\mathscr{A}^{(r)}(\tau)$ by means of the Airy scaling as in Corollary 1.2 and with the choice of $a$ above for $\rho=1$. Notice from (1.3) and using (1.10), this can also be expressed in terms of the matrix model with external potential:

$$
\begin{aligned}
\mathbb{P}\left(\mathscr{A}^{(r)}(\tau) \cap E=\emptyset\right) & :=\lim _{n \rightarrow \infty} \mathbb{P}_{B r}^{(0, \sqrt{n / 2})}\left(\text { all } x_{i}\left(\frac{1}{1+e^{-2 \tau / n^{1 / 3}}}\right) \in \frac{\sqrt{2 n}+\frac{E^{c}}{\sqrt{2} 1^{1 / 6}}}{2 \cosh \left(\tau / n^{1 / 3}\right)}\right) \\
& =\lim _{n \rightarrow \infty} \mathbb{P}_{n}\left(\sqrt{n} e^{\tau / n^{1 / 3}} ; 2 \sqrt{n}+\frac{E^{c}}{n^{1 / 6}}\right) .
\end{aligned}
$$

In a similar way, one defines the joint probability of $\mathscr{A}^{(r)}(\tau)$ for any number of times. The next section deals with this limit expressed in terms of a Fredholm determinant.

\section{The existence of the limit to the $r$-Airy kernel}

The first part of this section deals with a sketch of the proof of Theorem 0.1; details and rigor can be found in [8] and [29]. In the second part, the $r$-Airy kernel (1.14) will be expanded for large time $\tau$.

Proof of Theorem 0.1. From the explicit Brownian motion transition probability, one shows (see Johansson [19] and Tracy-Widom [28]) 


$$
\mathbb{P}_{B r}^{0 a}\left(\operatorname{all} x_{j}(t) \in E\right)=\operatorname{det}\left(I-H_{n}^{(r)}\right)_{L^{2}\left(E^{c}\right)}
$$

where

$H_{n}^{(r)}(x, y) d y=$

$$
-\frac{d y}{2 \pi^{2}(1-t)} \int_{\mathscr{D}} d z \int_{\Gamma_{L}} d w e^{-\frac{t z^{2}}{1-t}+\frac{2 x z}{1-t}+\frac{t w^{2}}{1-t}-\frac{2 w y}{1-t}}\left(\frac{w}{z}\right)^{n-r}\left(\frac{w-a}{z-a}\right)^{r} \frac{1}{w-z} ;
$$

$\mathscr{D}$ is a closed contour containing the points 0 and $a$, which is to the left of the line $\Gamma_{L}:=L+i \mathbb{R}$ by picking $L$ large enough. So, $\mathfrak{R}(w-z)>0$. Consider now an arbitrary point $(y, t)$ on the curve $\mathscr{C}$, parametrized by 2.2$)$ and the point $(\rho \sqrt{n / 2}, 1)$, which is the point of intersection of the tangent to $\mathscr{C}$ at $(y, t)$ with the axis $(t=1)$; as pointed out before, it is convenient to parametrize $\rho$ by $\rho=e^{-\sigma}$ and thus

$$
(y, t)=\left(\frac{\rho \sqrt{2 n}}{1+\rho^{2}}, \frac{1}{1+\rho^{2}}\right)=\left(\frac{\sqrt{2 n}}{2 \cosh (\sigma)}, \frac{1}{1+e^{-2 \sigma}}\right) \in \mathscr{C} .
$$

Consider the Brownian motions with $r$ outliers forced to a point $\rho_{0} \sqrt{n / 2}$ with $0<\rho_{0} \leq \rho$ at time $t=1$; here also parametrize $\rho_{0}$ by $\rho_{0}=e^{-\tau_{0}}$ and set $\alpha=$ $\rho_{0} / \rho=e^{\sigma-\tau_{0}}$.

The main issue is to compute the following limit, for $0<\rho_{0} \leq \rho$

$$
\lim _{n \rightarrow \infty} \mathbb{P}_{B r}^{\left(0, \rho_{0} \sqrt{n / 2}\right)}\left(\operatorname{all} x_{i}\left(\frac{1}{1+e^{-2\left(\sigma+\frac{\tau}{n^{1 / 3}}\right)}}\right) \in \frac{\sqrt{2 n}+\frac{E^{c}}{\sqrt{2} n^{1 / 6}}}{2 \cosh \left(\sigma+\frac{\tau}{n^{1 / 3}}\right)}\right),
$$

which dictates the space- and time-scale to be used in the kernel $H_{n}^{(r)}$ for large $n$. Let $t$ and $y$ be the time and space variables for the Brownian motion, which in terms of the new time and space scale $\mathscr{L}$ reads:

$$
\mathscr{L}: \quad t=\frac{1}{1+e^{-2\left(\sigma+\tau / n^{1 / 3}\right)}}, \quad x=\frac{\sqrt{2 n}+\frac{u}{\sqrt{2} n^{1 / 6}}}{2 \cosh \left(\sigma+\frac{\tau}{n^{1 / 3}}\right)}, \quad y=\frac{\sqrt{2 n}+\frac{v}{\sqrt{2} n^{1 / 6}}}{2 \cosh \left(\sigma+\frac{\tau}{n^{1 / 3}}\right)},
$$

with target point $a=\rho_{0} \sqrt{n / 2}=e^{-\tau_{0}} \sqrt{n / 2}$. Putting this rescaling in the integral (2.1) suggests changes of integration variables

$$
z:=\tilde{z} \sqrt{\frac{n}{2}} e^{-\sigma-\tau / n^{1 / 3}} \text { and } w:=\tilde{w} \sqrt{\frac{n}{2}} e^{-\sigma-\tau / n^{1 / 3}}
$$

in the integral $(2.1)$; the exponential will contain a function $F(z)$, with Taylor series at $z=1$ :

$$
F(z)=\frac{z^{2}}{2}-2 z+\log z=F(1)+\frac{1}{3}(z-1)^{3}+O(z-1)^{4} .
$$

Also set $\zeta:=1+\frac{\gamma}{n^{1 / 3}}$ for some parameter $\gamma$ and

$$
Z_{n}=\left\{\begin{array}{ll}
n^{-r / 3} e^{n F(1)} & \text { for } \quad \alpha=1 \\
(1-\alpha)^{r} e^{n F(1)} & \text { for } \quad 0<\alpha<1
\end{array} .\right.
$$


Then, using,

$$
\frac{1}{n^{1 / 3}(\tilde{w}-\tilde{z})}=\int_{0}^{\infty} e^{-y n^{1 / 3}(\tilde{w}-\tilde{z})} d y, \text { for } \Re(\tilde{w}-\tilde{z})>0
$$

one checks 7 , using the rescaling 2.3 :

$$
\begin{aligned}
&\left.H_{n}^{(r)}(x, y) d y\right|_{\mathscr{L}} \\
&=-\left(\frac{n^{1 / 3}}{2 \pi}\right)^{2} d v \int_{\mathscr{D}} d \tilde{z} \int_{\Gamma_{L}} d \tilde{w} e^{\left(-\frac{n}{2} \tilde{z}^{2}+\tilde{z}\left(2 n+n^{1 / 3} u\right)\right)} e^{-\left(-\frac{n}{2} \tilde{w}^{2}+\tilde{w}\left(2 n+n^{1 / 3} v\right)\right)} \\
&\left(\frac{\tilde{w}}{\tilde{z}}\right)^{n}\left(\frac{\tilde{w}-\alpha e^{\tau / n^{1 / 3}}}{\tilde{w}}\right)^{r}\left(\frac{\tilde{z}-\alpha e^{\tau / n^{1 / 3}}}{\tilde{z}}\right)^{-r} \frac{1}{n^{1 / 3}(\tilde{w}-\tilde{z})} \\
&=-e^{\zeta n^{1 / 3}(u-v)} d v \int_{0}^{\infty} d y \frac{Z_{n} n^{1 / 3}}{2 \pi} \int_{\mathscr{D}} d \tilde{z} e^{-n F(\tilde{z})}\left(\frac{\tilde{z}}{\tilde{z}-\alpha e^{\tau / n^{1 / 3}}}\right)^{r} e^{n^{1 / 3}(u+y)(\tilde{z}-\zeta)} \\
& \frac{n^{1 / 3}}{2 \pi Z_{n}} \int_{\Gamma_{L}} d \tilde{w} e^{n F(\tilde{w})}\left(\frac{\tilde{w}}{\tilde{w}-\alpha e^{\tau / n^{1 / 3}}}\right)^{-r} e^{-n^{1 / 3}(v+y)(\tilde{w}-\zeta)} .
\end{aligned}
$$

Conjugating the kernel, which leaves invariant the Fredholm determinant, one finds

$$
\left.e^{n^{1 / 3}(v-u)} H_{n}^{(r)}(x, y)\right|_{\mathscr{L}} d y=-d v e^{\gamma(u-v)} \int_{0}^{\infty} d y \mathscr{I}_{\tau}^{(n)}(u+y) \mathscr{J}_{\tau}^{(n)}(v+y)
$$

with

$$
\begin{aligned}
& \mathscr{I}_{\tau}^{(n)}(x)=\frac{n^{1 / 3} Z_{n}}{2 \pi} \int_{\mathscr{D}} d \tilde{z} e^{-n F(\bar{z})}\left(\frac{\tilde{z}}{\tilde{z}-\alpha e^{\tau / n^{1 / 3}}}\right)^{r} e^{n^{1 / 3} x(\tilde{z}-\zeta)} \\
& \mathscr{J}_{\tau}^{(n)}(y)=\frac{n^{1 / 3}}{2 \pi Z_{n}} \int_{\Gamma_{L}} d \tilde{w} e^{n F(\tilde{w})}\left(\frac{\tilde{w}}{\tilde{w}-\alpha e^{\tau / n^{1 / 3}}}\right)^{-r} e^{-n^{1 / 3} y(\tilde{w}-\zeta)}
\end{aligned}
$$

${ }^{7}$ by elementary computation

$$
\frac{t}{1-t}=e^{2\left(\sigma+\frac{\tau}{n^{1 / 3}}\right)}, \quad \frac{d y}{1-t} \frac{d z d w}{w-z}=\frac{n^{\frac{1}{3}} d \tilde{z} d \tilde{w} d v}{\tilde{w}-\tilde{z}}, \quad \frac{2 x z}{1-t}=\left(2 n+u n^{\frac{1}{3}}\right) \tilde{z}, \quad \frac{t z^{2}}{1-t}=\frac{n}{2} \tilde{z}^{2}
$$


Using the Taylor series (2.4) for $F(z)$ and the value (2.5) for $Z_{n}$, one is led naturally to pick a new variable $u$ such that $z-1=\frac{u}{n^{1 / 3}}$. Then

$$
\begin{aligned}
& n^{1 / 3} Z_{n} d \tilde{z} e^{-n F(\tilde{z})}\left(\frac{\tilde{z}}{\tilde{z}-\alpha e^{\tau / n^{1 / 3}}}\right)^{r} e^{n^{1 / 3} x(\tilde{z}-\zeta)} \\
& =n^{1 / 3} Z_{n} \frac{d u}{n^{1 / 3}}\left(e^{-n F(1)-\frac{u^{3}}{3}}+O\left(\frac{1}{n^{1 / 3}}\right)\right) \\
& \qquad\left(\frac{u+n^{1 / 3}}{u+n^{1 / 3}-\alpha\left(1+\frac{\tau}{n^{1 / 3}}+\ldots\right) n^{1 / 3}}\right)^{r} e^{x(u-\gamma)} \\
& = \begin{cases}e^{-x \gamma} d u e^{-\frac{u^{3}}{3}+x u}\left(\frac{1}{u-\tau}\right)^{r}+\text { lower order terms } & \text { for } \quad \alpha=1 \\
e^{-x \gamma} d u e^{-\frac{u^{3}}{3}+x u}+\text { lower order terms } & \text { for } \quad 0<\alpha<1 .\end{cases}
\end{aligned}
$$

Upon remembering the definition of the functions $A_{r}^{ \pm}(x, \tau)$ and the Airy function $A(x)$ and also the fact that $\alpha=\rho_{0} / \rho$, the rigorous saddle point argument, given in [8, 29], yields

$$
\begin{aligned}
& \lim _{n \rightarrow \infty} \mathscr{I}_{\tau}^{(n)}(x)= \\
& \left\{\begin{aligned}
& \frac{1}{2 \pi} \int_{\mathscr{D}^{\prime}} \frac{1}{(z-\tau)^{r}} e^{-\frac{z^{3}}{3}} e^{x(z-\gamma)} d z=\mathscr{I}_{\tau}(x):=e^{-x \gamma} A_{r}^{-}(x, \tau) \text { for } \quad \rho_{0}=\rho \\
& \frac{1}{2 \pi} \int_{\mathscr{D}^{\prime}} e^{-\frac{z^{3}}{3}} e^{x(z-\gamma)} d z=\mathscr{I}(x):=e^{-x \gamma} A(x) \\
& \text { for } 0<\rho_{0}<\rho
\end{aligned}\right. \\
& \lim _{n \rightarrow \infty} \mathscr{J}_{\tau}^{(n)}(y)= \\
& \left\{\begin{aligned}
\frac{1}{2 \pi} \int_{\mathscr{D}^{\prime \prime}}(z-\tau)^{r} e^{\frac{z^{3}}{3}} e^{-y(z-\gamma)} d z= & \mathscr{J}_{\tau}(y):=-e^{y \gamma} A_{r}^{+}(y, \tau), \\
& \text { for } \quad \rho_{0}=\rho \\
\frac{1}{2 \pi} \int_{\mathscr{D}^{\prime \prime}} e^{\frac{z^{3}}{3}} e^{-y(z-\gamma)} d z=\mathscr{J}(y) & :=-e^{y \gamma} A(y), \\
& \text { for } \quad 0<\rho_{0}<\rho
\end{aligned}\right.
\end{aligned}
$$

where $\mathscr{D}^{\prime}$ is a contour running from $\infty e^{4 i \pi / 3}$ to $\infty e^{2 i \pi / 3}$, with an indentation to the right of $\tau$, such that $\tau$ is to the left of the contour, and where $\mathscr{D}^{\prime \prime}$ is a contour running from $\infty e^{-i \pi / 3}$ to $\infty e^{i \pi / 3}$, with an indentation to the right of $\tau$, such that $\tau$ lies also to the left of the contour. Upon rotating the two contours and deforming $\mathscr{D}^{\prime \prime}$ slightly, since the integrand is pole-free, one gets the final identities in the equations above. 
Therefore $\mathbb{P}\left(\sup \mathscr{A}^{(r)}(\tau) \cap E=\emptyset\right)=\operatorname{det}\left(I-K_{\tau}^{(r)}\right)_{E}$, with

$$
\begin{aligned}
& K_{\tau}^{(r)}(u, v) d v=\left.\lim _{n \rightarrow \infty} e^{n^{1 / 3}(v-u)} H_{n}^{(r)}(x, y) d y\right|_{\mathscr{L}} \\
& =\left\{\begin{array}{lr}
d v \int_{0}^{\infty} d w A_{r}^{-}(u+w, \tau) A_{r}^{+}(v+w, \tau) & \text { for } \rho_{0}=\rho \\
d v \int_{0}^{\infty} d w A(u+w, \tau) A(v+w, \tau) & \text { for } 0<\rho_{0}<\rho .
\end{array}\right.
\end{aligned}
$$

Baik [9] has shown that the Fredholm determinant of the $r$-Airy kernel is a probability distribution, i.e.,

$$
\lim _{x \rightarrow \pm \infty} \operatorname{det}\left(I-K_{\tau}^{(r)}\right)_{(x, \infty)}=\left\{\begin{array}{c}
1 \\
0 .
\end{array}\right.
$$

This establishes Theorem 0.1

Remark 2.1. In this section one lets $\tau \rightarrow-\infty$, which implies that $-i \tau$ remains above the contour $C$ and is thus compatible with the contour mentioned above. Letting $\tau \rightarrow+\infty$ would require a drastic change of the functions $A_{r}^{ \pm}$.

The next statement concerns the asymptotic behavior of the $r$-Airy kernel for $\tau \rightarrow-\infty$,

$$
K_{\tau}^{(r)}(u, v)=\int_{0}^{\infty} d w A_{r}^{-}(u+w ; \tau) A_{r}^{+}(v+w ; \tau)
$$

where (remember)

$$
A_{r}^{ \pm}(u ; \tau)=\int_{C} e^{\frac{1}{3} i a^{3}+i a u}(\mp i a-\tau)^{ \pm r} \frac{d a}{2 \pi}
$$

where $C$ is a contour running from $\infty e^{5 i \pi / 6}$ to $\infty e^{i \pi / 6}$, such that $-i \tau$ lies above the contour. This limit is compatible with the contour $C$ appearing in the definition of the functions $A_{r}^{ \pm}$, since then $-i \tau$ remains above the contour $C$, as required.

Lemma 2.2. Given the "initial condition"

$$
\lim _{\tau \rightarrow-\infty} K_{\tau}^{(r)}(u, v)=K^{(0)}(u, v):=\frac{A(u) A^{\prime}(v)-A^{\prime}(u) A(v)}{u-v}=\text { "Airy kernel", }
$$

the kernel $K^{(r)}(u, v)$ behaves asymptotically for $\tau \rightarrow-\infty$, as

$$
K^{(r)}(u, v)=K_{0}+\frac{K_{1}^{(r)}}{\tau}+\frac{K_{2}^{(r)}}{\tau^{2}}+\frac{K_{3}^{(r)}}{\tau^{3}}+\ldots,
$$


where ${ }^{8}$ for $n \geq 1$,

$$
\begin{aligned}
K_{n}^{(r)}(u, v)= & -\left.\frac{r^{n}}{n !}\left(\frac{\partial}{\partial w}\right)^{n-1} A(u+w) A(v+w)\right|_{w=0} \\
& -\left.\frac{r^{n-1}}{2(n-2) !}\left(\frac{\partial}{\partial w}\right)^{n-2}\left(A^{\prime}(u+w) A(v+w)-A(u+w) A^{\prime}(v+w)\right)\right|_{w=0} \\
& -\left.\frac{r^{n-2}}{(n-3) !}\left(\begin{array}{c}
\frac{3 n-1}{24}\left(\frac{\partial}{\partial w}\right)^{n-1} A(u+w) A(v+w) \\
-\frac{n-1}{2}\left(\frac{\partial}{\partial w}\right)^{n-3} A^{\prime}(u+w) A^{\prime}(v+w)
\end{array}\right)\right|_{w=0} \\
& +(\text { polynomial of degree } n-3 \text { in } r) .
\end{aligned}
$$

Although the kernel $K_{\tau}^{(r)}(u, v)$ involves integration, the terms $K_{i}^{(r)}(u, v)$ in the expansion never involve integration, they are quadratic in the Airy function and its derivatives; also the $K_{i}^{(r)}(u, v)$ are polynomials in $r$ of degree $i$, divisible by $r$, with alternately symmetric and skew-symmetric coefficients in $u$ and $v$, the top coefficient being symmetric.

Proof. In order to expand the kernel 0.9 with regard to $\tau$ for $\tau \rightarrow-\infty$, set the expressions (2.10) into the kernel (0.9), which then becomes a triple integral. Set $\alpha=i a$ and $\beta=i b$ and consider the following Taylor expansions about $\tau=-\infty$,

$$
\begin{aligned}
\frac{1}{(\alpha-\tau)^{r}(-\beta-\tau)^{-r}} & =\left(1+\frac{\frac{\alpha+\beta}{\tau}}{1-\frac{\alpha}{\tau}}\right)^{r} \\
& =1+\sum_{n=1}^{\infty} \frac{1}{\tau^{n}} \sum_{j=0}^{\left[\frac{n-1}{2}\right]}\left(\begin{array}{c}
(r(\alpha+\beta))^{n-2 j} Q_{2 j}(\alpha, \beta) \\
+(r(\alpha+\beta))^{n-2 j-1}(\alpha-\beta) \tilde{Q}_{2 j}(\alpha, \beta)
\end{array}\right) \\
& \left.=1+\sum_{n=1}^{\infty} \frac{1}{\tau^{n}}\left(\begin{array}{l}
\frac{(r(\alpha+\beta))^{n}}{n !}+\frac{(r(\alpha+\beta))^{n-1}(\alpha-\beta)}{2(n-2) !} \\
+\frac{(r(\alpha+\beta))^{n-2}}{(n-3) !}\left(\frac{(3 n-1)(\alpha+\beta)^{2}}{24}-\frac{(n-1) \alpha \beta}{2}\right.
\end{array}\right)+\ldots\right)
\end{aligned}
$$

where $Q_{2 j}$ and $\tilde{Q}_{2 j}$ are symmetric homogeneous polynomials of degree $2 j$ in the arguments, since the first expression is invariant under the involution $r \mapsto-r$ and $\alpha \mapsto-\beta$. The coefficients of $1 / \tau^{n}$ are divisible by $r$, for the simple reason that for $r=0$, the expression above equals 1 .

Also notice multiplication by $i a$ of the integrand in the kernel (0.9),

$$
\begin{aligned}
K_{\tau}^{(r)}(u, v) & =\int_{0}^{\infty} d w \int_{C} e^{\frac{1}{3} i a^{3}+i a(w+u)}\left(\frac{1}{i a-\tau}\right)^{r} \frac{d a}{2 \pi} \int_{C} e^{\frac{1}{3} i b^{3}+i b(w+v)}(-i b-\tau)^{r} \frac{d b}{2 \pi} \\
& =\int_{0}^{\infty} d w \int_{C} \int_{C} \frac{d a d b}{4 \pi^{2}} e^{\frac{1}{3} i b^{3}+i b(w+v)} e^{\frac{1}{3} i a^{3}+i a(w+u)}\left(1+\frac{r}{\tau}(i a+i b)+\ldots\right)
\end{aligned}
$$

\footnotetext{
${ }^{8}$ Whenever $\partial / \partial w$ appears with a negative exponent in the formula below, it is set $=0$.
} 
can be realized by taking $\frac{\partial K^{(0)}}{\partial u}$ and similarly multiplication of the integrand by $i b$ is realized by taking $\frac{\partial K^{(0)}}{\partial v}$; thus we have the following recipe

$$
\begin{aligned}
i a & \leftrightarrow \frac{\partial K^{(0)}}{\partial u}=\int_{0}^{\infty} d w A^{\prime}(u+w) A(v+w) \\
i b & \leftrightarrow \frac{\partial K^{(0)}}{\partial v}=\int_{0}^{\infty} d w A(u+w) A^{\prime}(v+w),
\end{aligned}
$$

and so in particular,

$$
\begin{gathered}
(i a)^{k_{1}}(i b)^{k_{2}}(i a+i b)^{n} \Leftrightarrow \int_{0}^{\infty}\left(\frac{\partial}{\partial u}+\frac{\partial}{\partial v}\right)^{n}\left(\frac{\partial}{\partial u}\right)^{k_{1}}\left(\frac{\partial}{\partial v}\right)^{k_{2}} A(u+w) A(v+w) d w \\
=-\left.\left(\frac{\partial}{\partial w}\right)^{n-1} A^{\left(k_{1}\right)}(u+w) A^{\left(k_{2}\right)}(v+w)\right|_{w=0} .
\end{gathered}
$$

Notice that, since $i a+i b$ factors out of every term in the expansion (2.11, the kernels obtained never contain integration. In addition, since $i a-i b$ factors out of every other term, every other term in $K_{i}^{(r)}(u, v)$ must be skew; in particular it vanishes for $u=v$. One then reads off the $K_{i}^{(r)}(u, v)$ 's from the expansion 2.11) and the recipe above, upon using occasionally the differential equation $A^{\prime \prime}(x)=x A(x)$ for the Airy function, thus ending the proof of Lemma 2.2.

Remark 2.3. As an example, we give explicit expressions for the first few $K_{i}^{(r)}(u, v)$ 's:

$$
\begin{aligned}
K_{0}^{(r)}(u, v)= & K^{(0)}(u, v)=\frac{A(u) A^{\prime}(v)-A^{\prime}(u) A(v)}{u-v} \\
K_{1}^{(r)}(u, v)= & -r A(u) A(v) \\
K_{2}^{(r)}(u, v)= & -\frac{r^{2}}{2}\left(A^{\prime}(u) A(v)+A(u) A^{\prime}(v)\right)+\frac{r}{2}(u-v) K^{(0)}(u, v) \\
K_{3}^{(r)}(u, v)= & -\frac{r^{3}}{6}\left(A^{\prime \prime}(u) A(v)+2 A^{\prime}(u) A^{\prime}(v)+A(u) A^{\prime \prime}(v)\right)+\frac{r^{2}}{2}(v-u) A(u) A(v) \\
& -\frac{r}{3}\left(A^{\prime \prime}(u) A(v)+A(u) A^{\prime \prime}(v)-A^{\prime}(u) A^{\prime}(v)\right) .
\end{aligned}
$$

In order to find the PDE for the transition probability, one will need an estimate on how the actual transition probability for the finite problem converges for $n \rightarrow \infty$. This will be used in 6.3.

Corollary 2.4. For $x \in \mathbb{R}$ sufficiently large, one has for some constant $C>0$,

$$
\begin{aligned}
\log \mathbb{P}_{B r}^{\left(0, \rho_{0} \sqrt{\frac{n}{2}}\right)} & \left(\text { all } x_{i}\left(\frac{1}{1+e^{-2\left(\tau_{0}+\frac{\tau}{n^{1 / 3}}\right)}}\right) \leq \frac{\sqrt{2 n}+\frac{x}{\sqrt{2} n^{1 / 6}}}{2 \cosh \left(\tau_{0}+\frac{\tau}{n^{1 / 3}}\right)}\right) \\
& -\log \mathbb{P}\left(\sup \mathscr{A}^{(r)}(\tau) \leq x\right) \mid \leq C n^{-1 / 3} .
\end{aligned}
$$


Proof. For any trace class operators $K_{n}$ and $K_{\infty}$, set ${ }^{9}$

$$
M:=\max \left(\left\|K_{n}\right\|,|| K_{\infty} \|\right) .
$$

Then assuming $M<1$, one checks, using $K_{n}^{i}-K_{\infty}^{i}=\sum_{\ell=0}^{i-1} K_{\infty}^{\ell}\left(K_{n}-K_{\infty}\right) K_{n}^{i-1-\ell}$ and $\left\|\left.A B\right|_{\text {tr }} \leq\right\| A\|\| B \|_{\text {tr }}$ and $\|A B\|_{\text {tr }} \leq\|A\|_{\text {tr }}\|B\|$,

(2.14) $\left|\log \operatorname{det}\left(I-K_{n}\right)-\log \operatorname{det}\left(I-K_{\infty}\right)\right|=\left|\operatorname{tr}\left(\log \left(I-K_{n}\right)-\log \left(I-K_{\infty}\right)\right)\right|$

$$
\begin{aligned}
& \leq \sum_{1}^{\infty}\left|\operatorname{tr}\left(\frac{K_{n}^{i}-K_{\infty}^{i}}{i}\right)\right| \\
& \leq \sum_{1}^{\infty} \frac{1}{i}\left\|K_{n}^{i}-K_{\infty}^{i}\right\|_{\operatorname{tr}} \\
& \leq\left\|K_{n}-K_{\infty}\right\|_{\operatorname{tr}} \sum_{0}^{\infty} M^{i} \\
& \leq \frac{\left\|K_{n}-K_{\infty}\right\|_{\operatorname{tr}}}{1-M} .
\end{aligned}
$$

Setting (see notation (2.6)

$$
\begin{aligned}
K_{n}(u, v) & :=\int_{0}^{\infty} d y \mathscr{I}_{\tau}^{(n)}(u+y) \mathscr{J}_{\tau}^{(n)}(v+y) \\
K_{\infty}(u, v) & :=e^{(v-u) \gamma} \int_{0}^{\infty} d y A_{r}^{-}(u+y, \tau) A_{r}^{+}(v+y, \tau),
\end{aligned}
$$

one checks

$$
\begin{aligned}
& \left|K_{n}(u, v)-K_{\infty}(u, v)\right| \\
& \leq\left|\int_{0}^{\infty} d y\left(\mathscr{I}_{\tau}^{(n)}(u+y)-e^{-(u+y) \gamma} A_{r}^{-}(u+y, \tau)\right) \mathscr{J}_{\tau}^{(n)}(v+y)\right| \\
& \quad+\left|\int_{0}^{\infty} d y e^{-(u+y) \gamma} A_{r}^{-}(u+y, \tau)\left(\mathscr{J}_{\tau}^{(n)}(v+y)-e^{(v+y) \gamma} A_{r}^{+}(v+y, \tau)\right)\right| .
\end{aligned}
$$

An argument similar to the one of Baik-BenArous-Péché [8, 29] shows that for given $x_{0} \in \mathbb{R}$, there are constants $C>0, N>0$,

$$
\begin{aligned}
\left|\mathscr{I}_{\tau}^{(n)}(x)-e^{-x \gamma} A_{r}^{-}(x, \tau)\right| & \leq \frac{C e^{-c x}}{n^{1 / 3}} \\
\left|\mathscr{J}_{\tau}^{(n)}(x)-e^{x \gamma} A_{r}^{+}(x, \tau)\right| & \leq \frac{C e^{-c x}}{n^{1 / 3}}, \text { for } n \geq N \text { and } x \geq x_{0} .
\end{aligned}
$$

\footnotetext{
${ }^{9}$ Define the three norms on a Hilbert space: the sup, the trace and the Hilbert-Schmidt norms, with $\|T\| \leq\|T\|_{H S} \leq\|T\|_{\text {tr }}$ :$$
\|T\|=\sup _{v \in \mathscr{H}} \frac{|T v|}{|v|}=\sup \left|\lambda_{i}\right|,\|T\|_{\operatorname{tr}}=\operatorname{Tr}\left(T^{*} T\right)^{1 / 2}=\sum\left|\lambda_{i}\right|,\|T\|_{H S}=\left(\operatorname{Tr} T^{*} T\right)^{1 / 2}=\left(\sum\left|\lambda_{i}\right|^{2}\right)^{1 / 2} .
$$ 
Viewing the functions in the integrals on the right hand side as kernels representing Hänkel-like integral operators on $\left(x_{0}, \infty\right)$, one has, using the inequality $\|A B\|_{\text {tr }} \leq$ $\left.\|A\|_{H S}|| B\right|_{H S}$ for Hilbert-Schmidt operators,

$$
\begin{aligned}
\left\|K_{n}-K_{\infty}\right\|_{\operatorname{tr}} \leq & \left\|\mathscr{I}_{\tau}^{(n)}(u+y)-e^{-(u+y) \gamma_{A}}(u+y, \tau)\right\|_{H S}\left\|\mathscr{J}_{\tau}^{(n)}(v+y)\right\|_{H S} \\
& +\left\|e^{-(u+y) \gamma_{r}} A_{r}^{-}(u+y, \tau)\right\|_{H S}\left\|\mathscr{J}_{\tau}^{(n)}(v+y)-e^{(v+y) \gamma_{A}} A_{r}^{+}(v+y, \tau)\right\|_{H S} \\
\leq & 2 C^{\prime} n^{-1 / 3}
\end{aligned}
$$

where

$$
C^{\prime}=\max \left(\left\|e^{-(u+y) \gamma_{A}} A_{r}^{-}(u+y, \tau)\right\|_{H S}, \sup _{n \geq N}\left\|\mathscr{J}_{\tau}^{(n)}(v+y)\right\|_{H S}\right) C e^{-c x_{0}} .
$$

Then, from (2.14), one has that

$$
\left|\log \operatorname{det}\left(I-K_{n}\right)-\log \operatorname{det}\left(I-K_{\infty}\right)\right| \leq \frac{2 C^{\prime}}{1-M} n^{-1 / 3} .
$$

Remembering the representation of the probabilities in the statement 2.13) in terms of Fredholm determinants establishes Corollary 2.4

\section{An integrable deformation of Gaussian random ensemble with external source and 3-component KP}

The connection between the Gaussian random ensemble with external source and the multi-component KP hierarchy is explained in [4] and [6]. The main ideas are sketched in this section. For the multicomponent KP hierarchy, see [30].

\subsection{Two sets of weights and the $p+q$-KP hierarchy}

Define two sets of weights

$$
\psi_{1}(x), \ldots, \psi_{q}(x) \text { and } \varphi_{1}(y), \ldots, \varphi_{p}(y), \text { with } x, y \in \mathbb{R},
$$

and deformed weights depending on time parameters $s_{\alpha}=\left(s_{\alpha 1}, s_{\alpha 2}, \ldots\right)(1 \leq \alpha \leq$ $q)$ and $t_{\beta}=\left(t_{\beta 1}, t_{\beta 2}, \ldots\right)(1 \leq \beta \leq p)$, denoted by

$$
\psi_{\alpha}^{-s}(x):=\psi_{\alpha}(x) e^{-\sum_{k=1}^{\infty} s_{\alpha k} x^{k}} \text { and } \varphi_{\beta}^{t}(y):=\varphi_{\beta}(y) e^{\sum_{k=1}^{\infty} t_{\beta k} y^{k}}
$$

That is, each weight goes with its own set of times. For each set of positive integers $^{10} m=\left(m_{1}, \ldots, m_{q}\right), n=\left(n_{1}, \ldots, n_{p}\right)$ with $|m|=|n|$, consider the determinant

$$
{ }^{10}|m|=\sum_{\alpha=1}^{q} m_{\alpha} \text { and }|n|=\sum_{\beta=1}^{p} n_{\beta} .
$$


of a moment matrix $T_{m n}$ of size $|m|=|n|$, composed of $p q$ blocks of sizes $m_{i} n_{j}$; the moments are taken with regard to a (not necessarily symmetric) inner product $\langle\cdot \mid \cdot\rangle$

$$
\begin{aligned}
& \tau_{m n}\left(s_{1}, \ldots, s_{q} ; t_{1}, \ldots, t_{p}\right) \\
& :=\operatorname{det}\left(\begin{array}{ccc}
\left(\left\langle x^{i} \psi_{1}^{-s}(x) \mid y^{j} \varphi_{1}^{t}(y)\right\rangle\right)_{\substack{0 \leq i<m_{1} \\
0 \leq j<n_{1}}} \ldots & \left(\left\langle x^{i} \psi_{1}^{-s}(x) \mid y^{j} \varphi_{p}^{t}(y)\right\rangle\right)_{\substack{0 \leq i<m_{1} \\
0 \leq j<n_{p}}} \\
\vdots & \vdots \\
\left(\left\langle x^{i} \psi_{q}^{-s}(x) \mid y^{j} \varphi_{1}^{t}(y)\right\rangle\right)_{\substack{0 \leq i<m_{q} \\
0 \leq j<n_{1}}} \ldots & \left(\left\langle x^{i} \psi_{q}^{-s}(x) \mid y^{j} \varphi_{p}^{t}(y)\right\rangle\right)_{\substack{0 \leq i<m_{q} \\
0 \leq j<n_{p}}}
\end{array}\right) .
\end{aligned}
$$

We now state a non-trivial Theorem involving a relationship between the determinants of the block moment matrices above, by increasing or decreasing the sizes of the blocks by one. Modifying the size $n_{\beta}$ in $n=\left(n_{1}, \ldots, n_{p}\right)$ by 1 is indicated by $n \mapsto n \pm e_{\beta}$, where $e_{\beta}=(0, \ldots, 0,1,0, \ldots, 0)$, with 1 at place $\beta$. The proof and many simple examples can be found in [6]:

Theorem 3.1. (Adler, van Moerbeke and Vanhaecke [6]) Then the block matrices $\tau_{m n}$ satisfy the $(p+q)$-KP hierarchy; to be precise, the functions $\tau_{m n}$ satisfy the bilinear relations 11

$$
\begin{gathered}
\sum_{\beta=1}^{p} \oint_{\infty}(-1)^{\sigma_{\beta}(n)} \tau_{m, n-e_{\beta}}\left(t_{\beta}-\left[z^{-1}\right]\right) \tau_{m^{*}, n^{*}+e_{\beta}}\left(t_{\beta}^{*}+\left[z^{-1}\right]\right) e^{\Sigma_{1}^{\infty}\left(t_{\beta k}-t_{\beta k}^{*} k^{k}\right.} z^{n_{\beta}-n_{\beta}^{*}-2} d z= \\
\sum_{\alpha=1}^{q} \oint_{\infty}(-1)^{\sigma_{\alpha}(m)} \tau_{m+e_{\alpha}, n}\left(s_{\alpha}-\left[z^{-1}\right]\right) \tau_{m^{*}-e_{\alpha}, n^{*}}\left(s_{\alpha}^{*}+\left[z^{-1}\right]\right) e^{\sum_{1}^{\infty}\left(s_{\alpha k}-s_{\alpha k}^{*}\right) z^{k}} z^{m_{\alpha}^{*}-m_{\alpha}-2} d z
\end{gathered}
$$

for all $m, n, m^{*}, n^{*}$ such that $\left|m^{*}\right|=\left|n^{*}\right|+1$ and $|m|=|n|-1$ and all $s, t, s^{*}, t^{*} \in \mathbb{C}^{\infty}$ and where $\sigma_{\alpha}(m)=\sum_{\alpha^{\prime}=1}^{\alpha}\left(m_{\alpha^{\prime}}-m_{\alpha^{\prime}}^{*}\right) \quad$ and $\quad \sigma_{\beta}(n)=\sum_{\beta^{\prime}=1}^{\beta}\left(n_{\beta^{\prime}}-n_{\beta^{\prime}}^{*}\right)$.

Computing the residues in the contour integrals above, the functions $\tau_{m n}$, with $|m|=|n|$, satisfy the following PDE's in terms of the Hirota symbol, defined in the

${ }^{11}$ The integrals are contour integrals along a small circle about $\infty$, with formal Laurent series as the integrand. Also, for $z \in \mathbb{C}$, we define $\left[z^{-1}\right]:=\left(\frac{z^{-1}}{1}, \frac{z^{-2}}{2}, \frac{z^{-3}}{3}, \ldots\right)$. For a given polynomial $p\left(t_{1}, t_{2}, \ldots\right)$, the Hirota symbol between functions $f=f\left(t_{1}, t_{2}, \ldots\right)$ and $g=g\left(t_{1}, t_{2}, \ldots\right)$ is defined by $p\left(\frac{\partial}{\partial t_{1}}, \frac{\partial}{\partial t_{2}}, \ldots\right) f \circ g:=\left.p\left(\frac{\partial}{\partial y_{1}}, \frac{\partial}{\partial y_{2}}, \ldots\right) f(t+y) g(t-y)\right|_{y=0}$. We also need the elementary Schur polynomials $\mathbf{s}_{\ell}$, defined by $e^{\sum_{1}^{\infty} t_{k} z^{k}}:=\sum_{k \geq 0} \mathbf{s}_{k}(t) z^{k}$ for $\ell \geq 0$ and $\mathbf{s}_{\ell}(t)=0$ for $\ell<0$; moreover, set $\mathbf{s}_{\ell}\left(\tilde{\partial}_{t}\right):=\mathbf{s}_{\ell}\left(\frac{\partial}{\partial t_{1}}, \frac{1}{2} \frac{\partial}{\partial t_{2}}, \frac{1}{3} \frac{\partial}{\partial t_{3}}, \ldots\right)$. 
footnote 11:

$$
\begin{aligned}
& \tau_{m n}^{2} \frac{\partial^{2}}{\partial t_{\beta, \ell+1} \partial t_{\beta^{\prime}, 1}} \log \tau_{m n}=\mathbf{s}_{\ell+2 \delta_{\beta \beta^{\prime}}}\left(\tilde{\partial}_{t_{\beta}}\right) \tau_{m, n+e_{\beta}-e_{\beta^{\prime}}} \circ \tau_{m, n+e_{\beta^{\prime}}-e_{\beta}} \\
& \tau_{m n}^{2} \frac{\partial^{2}}{\partial s_{\alpha, \ell+1} \partial s_{\alpha^{\prime}, 1}} \log \tau_{m n}=\mathbf{s}_{\ell+2 \delta_{\alpha \alpha^{\prime}}}\left(\tilde{\partial}_{s_{\alpha}}\right) \tau_{m+e_{\alpha^{\prime}}}-e_{\alpha}, n \\
& \tau_{m n}^{2} \frac{\partial^{2}}{\partial s_{\alpha, 1} \partial t_{\beta, \ell+1}} \log \tau_{m n}=-\mathbf{s}_{\ell}\left(\tilde{\partial}_{t_{\beta}}\right) \tau_{m+e_{\alpha}, n+e_{\beta}} \circ \tau_{m-e_{\alpha}, n-e_{\beta}} \\
& \tau_{m n}^{2} \frac{\partial^{2}}{\partial t_{\beta, 1} \partial s_{\alpha, \ell+1}} \log \tau_{m n}=-\mathbf{s}_{\ell}\left(\tilde{\partial}_{s_{\alpha}}\right) \tau_{m-e_{\alpha}, n-e_{\beta}} \circ \tau_{m+e_{\alpha}, n+e_{\beta}} .
\end{aligned}
$$

\subsection{Gaussian ensemble with external source}

Consider an ensemble of $n \times n$ Hermitian matrices with an external source, given by a diagonal matrix $A=\operatorname{diag}\left(a_{1}, \ldots, a_{n}\right)$ and a general potential $V(z)$, with density

$$
\mathbb{P}_{n}(M \in[M, M+d M])=\frac{1}{Z_{n}} e^{-\operatorname{Tr}(V(M)-A M)} d M .
$$

For a subset $E \subset \mathbb{R}$, the following probability can be transformed by the HarishChandra-Itzykson-Zuber formula, with $D:=\operatorname{diag}\left(z_{1}, \ldots, z_{n}\right)$,

$$
\Delta_{n}(z):=\prod_{1 \leq i<j \leq n}\left(z_{i}-z_{j}\right)
$$

and all distinct $a_{i}$,

$$
\begin{aligned}
\mathbb{P}_{n}(\text { spectrum } M \subset E) & =\frac{1}{Z_{n}} \int_{\mathscr{H}_{n}(E)} e^{-\operatorname{Tr}(V(M)-A M)} d M \\
& =\frac{1}{Z_{n}} \int_{E^{n}} \Delta_{n}^{2}(z) \prod_{1}^{n} e^{-V\left(z_{i}\right)} d z_{i} \int_{U(n)} e^{\operatorname{Tr} A U D U^{-1}} d U \\
& =\frac{1}{Z_{n}^{\prime}} \int_{E^{n}} \Delta_{n}^{2}(z) \prod_{1}^{n} e^{-V\left(z_{i}\right)} d z_{i} \frac{\operatorname{det}\left[e^{a_{i} z_{j}}\right]_{1 \leq i, j \leq n}}{\Delta_{n}(z) \Delta_{n}(a)} \\
& =\frac{1}{Z_{n}^{\prime \prime}} \int_{E^{n}} \Delta_{n}(z) \operatorname{det}\left[e^{-V\left(z_{j}\right)+a_{i} z_{j}}\right]_{1 \leq i, j \leq n} \prod_{1}^{n} d z_{i}
\end{aligned}
$$

with $a_{i} \neq a_{j}$ and the Vandermonde $\Delta_{n}(z)=\prod_{1 \leq i<j \leq n}\left(z_{i}-z_{j}\right)$. The formula remains valid in the limit, when some $a_{i}$ 's coincide, upon making differences of rows and dividing by the appropriate $\left(a_{i}-a_{j}\right)$ 's. In the following Proposition, we consider a general situation, of which 3.5 with $A=\operatorname{diag}(a, \ldots, a, 0, \ldots, 0)$ is a special case, by setting $\varphi^{+}=e^{a z}$ and $\varphi^{-}=1$. Consider the Vandermonde determinant $\Delta_{n}(x, y):=\Delta_{n}\left(x_{1}, \ldots, x_{k_{1}}, y_{1}, \ldots, y_{k_{2}}\right)$. Then we have the following (see [4]): 
Proposition 3.2. Given an arbitrary potential $V(z)$ and arbitrary functions $\varphi^{+}(z)$ and $\varphi^{-}(z)$, define $\left(n=k_{1}+k_{2}\right)$

$$
\begin{aligned}
&\left(\rho_{1}, \ldots, \rho_{n}\right):=e^{-V(z)}\left(\varphi^{+}(z), z \varphi^{+}(z), \ldots, z^{k_{1}-1} \varphi^{+}(z)\right. \\
&\left.\varphi^{-}(z), z \varphi^{-}(z), \ldots, z^{k_{2}-1} \varphi^{-}(z)\right) .
\end{aligned}
$$

We have

$$
\begin{aligned}
& \frac{1}{n !} \int_{E^{n}} \Delta_{n}(z) \operatorname{det}\left(\rho_{i}\left(z_{j}\right)\right)_{1 \leq i, j \leq n} \prod_{1}^{n} d z_{i} \\
= & \frac{1}{k_{1} ! k_{2} !} \int_{E^{n}} \Delta_{n}(x, y) \Delta_{k_{1}}(x) \Delta_{k_{2}}(y) \prod_{1}^{k_{1}} \varphi^{+}\left(x_{i}\right) e^{-V\left(x_{i}\right)} d x_{i} \prod_{1}^{k_{2}} \varphi^{-}\left(y_{i}\right) e^{-V\left(y_{i}\right)} d y_{i} \\
= & \operatorname{det}\left(\begin{array}{c}
\left(\int_{E} z^{i+j} \varphi^{+}(z) e^{-V(z)}\right) \\
0 \leq i \leq k_{1}-1 \\
0 \leq j \leq k_{1}+k_{2}-1 \\
\left(\int_{E} z^{i+j} \varphi^{-}(z) e^{-V(z)}\right) \\
0 \leq i \leq k_{2}-1 \\
0 \leq j \leq k_{1}+k_{2}-1
\end{array}\right)
\end{aligned}
$$

\subsection{Adding extra-variables $t, s u$, and $\beta$}

We add the extra-variables $t=\left(t_{1}, t_{2}, \ldots\right), s=\left(s_{1}, s_{2}, \ldots\right), u=\left(u_{1}, u_{2}, \ldots\right)$ and $\beta$ in the exponentials, as follows $\left(n=k_{1}+k_{2}\right)$,

$$
\begin{gathered}
V(z):=\frac{z^{2}}{2}-\sum_{1}^{\infty} t_{i} z^{i} \\
\varphi^{+}(z)=e^{a z+\beta z^{2}-\sum_{1}^{\infty} s_{i} z^{i}}, \varphi^{-}(z)=e^{-\sum_{1}^{\infty} u_{i} z^{i}} .
\end{gathered}
$$

The determinant of the moment matrix (3.2) with regard to the inner-product $\langle f, g\rangle=$ $\int_{E} f(z) g(z) e^{-z^{2} / 2} d z$, with $p=1, q=2, n_{1}=k_{1}+k_{2}, m_{1}=k_{1}, m_{2}=k_{2}$, and $\varphi_{1}(x)=1, \psi_{1}(y)=e^{a y+\beta y^{2}}, \psi_{2}(y)=1$ is the same as the determinant 3.6$)$, with the expressions $V(z)$ and $\varphi^{ \pm}(z)$ as in (3.7), and setting $s_{1 i}:=s_{i}, s_{2 i}=u_{i}, t_{1 i}=t_{i}$. 
Therefore by virtue of Theorem 3.1, the expression below satisfies the 3-KP hierarchy, since $p+q=3$, namely,

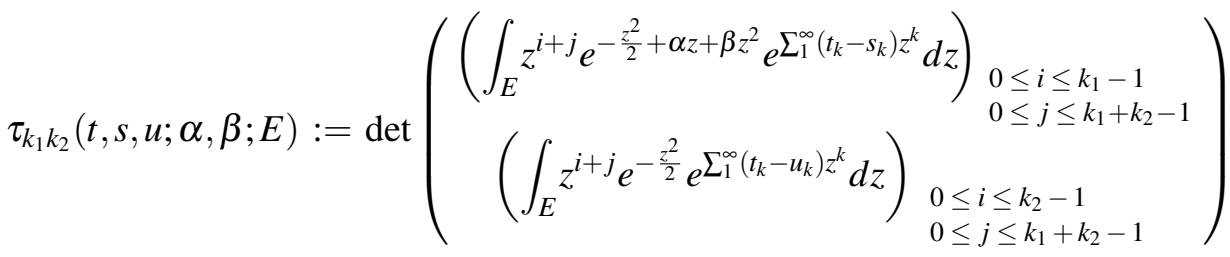

$$
\begin{aligned}
& =\frac{1}{k_{1} ! k_{2} !} \int_{E^{n}} \Delta_{n}(x, y) \prod_{j=1}^{k_{1}} e^{\sum_{1}^{\infty} t_{i} x_{j}^{i}} \prod_{j=1}^{k_{2}} e^{\sum_{1}^{\infty} t_{i} y_{j}^{i}} \\
& \left(\Delta_{k_{1}}(x) \prod_{j=1}^{k_{1}} e^{-\frac{x_{j}^{2}}{2}+\alpha x_{j}+\beta x_{j}^{2}} e^{-\sum_{1}^{\infty} s_{i} x_{j}^{i}} d x_{j}\right) \\
& \left(\Delta_{k_{2}}(y) \prod_{j=1}^{k_{2}} e^{-\frac{y_{j}^{2}}{2}} e^{-\sum_{1}^{\infty} u_{i} y_{j}^{i}} d y_{j}\right) .
\end{aligned}
$$

Corollary 3.3. The functions $\tau_{k_{1} k_{2}}(t, s, u)$ satisfy the identities

$$
\frac{\partial}{\partial t_{1}} \log \frac{\tau_{k_{1}+1, k_{2}}}{\tau_{k_{1}-1, k_{2}}}=\frac{\frac{\partial^{2}}{\partial t_{2} \partial s_{1}} \log \tau_{k_{1}, k_{2}}}{\frac{\partial^{2}}{\partial t_{1} \partial s_{1}} \log \tau_{k_{1}, k_{2}}},-\frac{\partial}{\partial s_{1}} \log \frac{\tau_{k_{1}+1, k_{2}}}{\tau_{k_{1}-1, k_{2}}}=\frac{\frac{\partial^{2}}{\partial t_{1} \partial s_{2}} \log \tau_{k_{1}, k_{2}}}{\frac{\partial^{2}}{\partial t_{1} \partial s_{1}} \log \tau_{k_{1}, k_{2}}}
$$

(3.10) $\frac{\partial}{\partial t_{1}} \log \frac{\tau_{k_{1}, k_{2}+1}}{\tau_{k_{1}, k_{2}-1}}=\frac{\frac{\partial^{2}}{\partial t_{2} \partial u_{1}} \log \tau_{k_{1}, k_{2}}}{\frac{\partial^{2}}{\partial t_{1} \partial u_{1}} \log \tau_{k_{1}, k_{2}}},-\frac{\partial}{\partial u_{1}} \log \frac{\tau_{k_{1}, k_{2}+1}}{\tau_{k_{1}, k_{2}-1}}=\frac{\frac{\partial^{2}}{\partial t_{1} \partial u_{2}} \log \tau_{k_{1}, k_{2}}}{\frac{\partial^{2}}{\partial t_{1} \partial u_{1}} \log \tau_{k_{1}, k_{2}}}$.

Proof. The bilinear identities (3.3) imply the PDE's (3.4) for

$$
\tau_{k_{1} k_{2}}(t, s, u):=\tau_{k_{1}, k_{2}, k_{1}+k_{2}}(t, s, u),
$$

(in the notation of (3.2), setting $m=\left(k_{1}, k_{2}\right)$ and $\left.n=k_{1}+k_{2}\right)$ expressed in terms of Hirota's symbol, for $j=0,1,2, \ldots$,

$$
\begin{aligned}
& \mathbf{s}_{j}\left(\tilde{\partial}_{t}\right) \tau_{k_{1}+1, k_{2}} \circ \tau_{k_{1}-1, k_{2}}=-\tau_{k_{1} k_{2}}^{2} \frac{\partial^{2}}{\partial s_{1} \partial t_{j+1}} \log \tau_{k_{1} k_{2}} \\
& \mathbf{s}_{j}\left(\tilde{\partial}_{s}\right) \tau_{k_{1}-1, k_{2}} \circ \tau_{k_{1}+1, k_{2}}=-\tau_{k_{1} k_{2}}^{2} \frac{\partial^{2}}{\partial t_{1} \partial s_{j+1}} \log \tau_{k_{1} k_{2}} \\
& \mathbf{s}_{j}\left(\tilde{\partial}_{t}\right) \tau_{k_{1}, k_{2}+1} \circ \tau_{k_{1}, k_{2}-1}=-\tau_{k_{1} k_{2}}^{2} \frac{\partial^{2}}{\partial u_{1} \partial t_{j+1}} \log \tau_{k_{1} k_{2}} \\
& \mathbf{s}_{j}\left(\tilde{\partial}_{u}\right) \tau_{k_{1}, k_{2}-1} \circ \tau_{k_{1}, k_{2}+1}=-\tau_{k_{1} k_{2}}^{2} \frac{\partial^{2}}{\partial t_{1} \partial u_{j+1}} \log \tau_{k_{1} k_{2}} .
\end{aligned}
$$


In particular for $j=0$, one finds the following expressions

$$
\begin{aligned}
& \frac{\partial^{2} \log \tau_{k_{1}, k_{2}}}{\partial t_{1} \partial s_{1}}=-\frac{\tau_{k_{1}+1, k_{2}} \tau_{k_{1}-1, k_{2}}}{\tau_{k_{1}, k_{2}}^{2}} \\
& \frac{\partial^{2} \log \tau_{k_{1}, k_{2}}}{\partial t_{1} \partial u_{1}}=-\frac{\tau_{k_{1}, k_{2}+1} \tau_{k_{1}, k_{2}-1}}{\tau_{k_{1}, k_{2}}^{2}}
\end{aligned}
$$

and another set of expressions for $j=1$. Then taking appropriate ratios of these expressions yields the formulae of Corollary 3.3 .

\section{Virasoro constraints}

Define the differential operators $\mathscr{B}_{m}$ involving the boundary points of the set $E$,

$$
\mathscr{B}_{m}:=\sum_{i=1}^{2 r} b_{i}^{m+1} \frac{\partial}{\partial b_{i}}, \quad \text { for } \quad E=\bigcup_{i=1}^{r}\left[b_{2 i-1}, b_{2 i}\right] \subset \mathbb{R},
$$

and the differential operators $\mathbb{V}_{-1}$ and $\mathbb{V}_{0}$ involving differentiation with respect to the auxiliary variables $t_{i}, s_{i}, u_{i}$ and $\beta$ :

$$
\begin{aligned}
\mathbb{V}_{-1}:= & -\frac{\partial}{\partial t_{1}}-2 \beta \frac{\partial}{\partial s_{1}}+\sum_{i \geq 2}\left(i t_{i} \frac{\partial}{\partial t_{i-1}}+i s_{i} \frac{\partial}{\partial s_{i-1}}+i u_{i} \frac{\partial}{\partial u_{i-1}}\right) \\
& +k_{1}\left(t_{1}-s_{1}\right)+k_{2}\left(t_{1}-u_{1}\right)+\alpha k_{1} \\
\mathbb{V}_{0}:= & -\frac{\partial}{\partial t_{2}}-2 \beta \frac{\partial}{\partial s_{2}}-\alpha \frac{\partial}{\partial s_{1}}+\sum_{i \geq 1}\left(i i_{i} \frac{\partial}{\partial t_{i}}+i s_{i} \frac{\partial}{\partial s_{i}}+i u_{i} \frac{\partial}{\partial u_{i}}\right) \\
& +k_{1}^{2}+k_{2}^{2}+k_{1} k_{2} .
\end{aligned}
$$

Theorem 4.1. The integral $\tau_{k_{1} k_{2}}(t, s, u ; \alpha, \beta ; E)$ defined in (3.8) satisfies :

$$
\mathscr{B}_{m} \tau_{k_{1} k_{2}}=\mathbb{V}_{m} \tau_{k_{1} k_{2}} \text { for } m=-1 \text { and } 0,
$$

with $\mathbb{V}_{m}$ as in 4.1 .

Proof. Let $I_{k_{1} k_{2}}(x, y)$ be the integrand of the integral $\tau_{k_{1} k_{2}}(t, s, u ; \alpha, \beta ; E)$ defined in (3.8):

$$
\tau:=\tau_{k_{1} k_{2}}(t, s, u ; \alpha, \beta ; E)=\int_{E^{n}} I_{k_{1} k_{2}}(x, y) \prod_{1}^{k_{1}} d x_{i} \prod_{1}^{k_{2}} d y_{j} .
$$

To obtain (4.1), one uses the fundamental Theorem of calculus and the commutation relation

$$
\sum_{1}^{n}\left(\frac{\partial}{\partial x_{i}} x_{i}-x_{i} \frac{\partial}{\partial x_{i}}\right)=n
$$

one uses the fact that for any Vandermonde,

$$
\sum_{1}^{k} \frac{\partial}{\partial x_{i}} \Delta\left(x_{1}, \ldots, x_{k}\right)=0
$$


and

$$
\sum_{1}^{k} x_{i} \frac{\partial}{\partial x_{i}} \Delta\left(x_{1}, \ldots, x_{k}\right)=\frac{n(n-1)}{2} \Delta\left(x_{1}, \ldots, x_{k}\right)
$$

Also one uses the fact that the sum of the $x_{i}$ and $y_{i}$ derivatives of the integrand $I_{k_{1}, k_{2}}$ translates into the $t_{i}, s_{i}$ and $u_{i}$-derivatives of $I_{k_{1}, k_{2}}$, i.e., the auxiliary parameters $t, s, u ; \alpha, \beta$ were precisely added for this very purpose! So we find:

$$
\begin{aligned}
\mathscr{B}_{-1} \tau & =\int_{E^{n}}\left(\sum_{1}^{k_{1}} \frac{\partial}{\partial x_{j}}+\sum_{1}^{k_{2}} \frac{\partial}{\partial y_{j}}\right) I_{k_{1}, k_{2}} \prod_{1}^{k_{1}} d x_{i} \prod_{1}^{k_{2}} d y_{j} \\
& =\int_{E^{n}} \mathbb{V}_{-1}\left(I_{k_{1}, k_{2}}\right) \prod_{1}^{k_{1}} d x_{i} \prod_{1}^{k_{2}} d y_{j} \\
& =\mathbb{V}_{-1}\left(\int_{E^{n}} I_{k_{1}, k_{2}} \prod_{1}^{k_{1}} d x_{i} \prod_{1}^{k_{2}} d y_{j}\right)=\mathbb{V}_{-1} \tau
\end{aligned}
$$

and

$$
\begin{aligned}
\mathscr{B}_{0} \tau & =\int_{E^{n}}\left(\sum_{1}^{k_{1}} \frac{\partial}{\partial x_{j}} x_{j}+\sum_{1}^{k_{1}} \frac{\partial}{\partial y_{j}} y_{j}\right) I_{k_{1}, k_{2}} \prod_{1}^{k_{1}} d x_{i} \prod_{1}^{k_{2}} d y_{j} \\
& =\int_{E^{n}} \mathbb{V}_{0}\left(I_{k_{1}, k_{2}}\right) \prod_{1}^{k_{1}} d x_{i} \prod_{1}^{k_{2}} d y_{j} \\
& =\mathbb{V}_{0}\left(\int_{E^{n}} I_{k_{1}, k_{2}} \prod_{1}^{k_{1}} d x_{i} \prod_{1}^{k_{2}} d y_{j}\right)=\mathbb{V}_{0} \tau
\end{aligned}
$$

establishing Theorem 4.1. Aother way of computing this has appeared in [2].

We also have the following identities, valid when acting on $\tau_{k_{1} k_{2}}(t, s, u ; \alpha, \beta ; E)$ :

$$
\frac{\partial}{\partial t_{n}}=-\frac{\partial}{\partial s_{n}}-\frac{\partial}{\partial u_{n}}
$$

$$
\frac{\partial}{\partial s_{1}}=-\frac{\partial}{\partial \alpha}, \frac{\partial}{\partial t_{1}}=\frac{\partial}{\partial \alpha}-\frac{\partial}{\partial u_{1}}, \frac{\partial}{\partial s_{2}}=-\frac{\partial}{\partial \beta}, \frac{\partial}{\partial t_{2}}=\frac{\partial}{\partial \beta}-\frac{\partial}{\partial u_{2}}
$$


Corollary 4.2. On the locus $\mathscr{L}=\{t=s=u=\beta=0\}$, the function $f:=\log \tau_{k_{1} k_{2}}(t, s, u ; \alpha, \beta ; E)$ satisfies the Virasoro constraints :

$$
\begin{array}{ll}
\frac{\partial f}{\partial s_{1}} & =-\frac{\partial f}{\partial \alpha}, \quad \frac{\partial f}{\partial s_{2}}=-\frac{\partial f}{\partial \beta} \\
\frac{\partial f}{\partial t_{1}} & =-\mathscr{B}_{-1} f+\alpha k_{1}, \quad \frac{\partial f}{\partial u_{1}}=\left(\mathscr{B}_{-1}+\frac{\partial}{\partial \alpha}\right) f-\alpha k_{1} \\
\frac{\partial f}{\partial t_{2}} & =\left(-\mathscr{B}_{0}+\alpha \frac{\partial}{\partial \alpha}\right) f+\left(k_{1}^{2}+k_{2}^{2}+k_{1} k_{2}\right) \\
\frac{\partial f}{\partial u_{2}} & =\left(\mathscr{B}_{0}-\alpha \frac{\partial}{\partial \alpha}+\frac{\partial}{\partial \beta}\right) f-\left(k_{1}^{2}+k_{2}^{2}+k_{1} k_{2}\right)
\end{array}
$$

$$
\begin{aligned}
\frac{\partial^{2} f}{\partial t_{1} \partial u_{1}} & =-\mathscr{B}_{-1}\left(\mathscr{B}_{-1}+\frac{\partial}{\partial \alpha}\right) f-k_{2} \\
\frac{\partial^{2} f}{\partial t_{1} \partial u_{2}} & =-\mathscr{B}_{-1}\left(\mathscr{B}_{0}-\alpha \frac{\partial}{\partial \alpha}+\frac{\partial}{\partial \beta}\right) f+2\left(\mathscr{B}_{-1}+\frac{\partial}{\partial \alpha}\right) f-2 \alpha k_{1} \\
\frac{\partial^{2} f}{\partial t_{2} \partial u_{1}} & =\left(-\mathscr{B}_{0}+\alpha \frac{\partial}{\partial \alpha}+1\right)\left(\mathscr{B}_{-1}+\frac{\partial}{\partial \alpha}\right) f-2 \alpha k_{1}
\end{aligned}
$$

$$
\begin{aligned}
\frac{\partial^{2} f}{\partial t_{1} \partial s_{1}} & =\mathscr{B}_{-1}\left(\frac{\partial}{\partial \alpha}\right) f-k_{1} \\
\frac{\partial^{2} f}{\partial t_{1} \partial s_{2}} & =\left(\mathscr{B}_{-1} \frac{\partial}{\partial \beta}-2 \frac{\partial}{\partial \alpha}\right) f \\
\frac{\partial^{2} f}{\partial t_{2} \partial s_{1}} & =\left(\mathscr{B}_{0}-\alpha \frac{\partial}{\partial \alpha}-1\right)\left(\frac{\partial}{\partial \alpha}\right) f .
\end{aligned}
$$

Proof. Upon dividing by $\tau$, equations (4.4) are a direct consequence of (4.1) and (4.3), when evaluated on the locus $\mathscr{L}$. To derive equations (4.5) and (4.6), we use the fact that the boundary operators

$$
\mathscr{B}_{m}=\sum_{j=1}^{2 r} b_{j}^{m+1} \frac{\partial}{\partial b_{j}}
$$

commute with pure time-differential operators. For example, the calculation of the first equation in (4.5) goes as follows. We know from (4.1) and (4.3) that :

$$
-\mathscr{B}_{-1} f=\left(\frac{\partial f}{\partial t_{1}}-\alpha k_{1}+L_{1} f+\ell_{1}\right),\left(\mathscr{B}_{-1}+\frac{\partial}{\partial \alpha}\right) f=\left(\frac{\partial f}{\partial u_{1}}+\alpha k_{1}+L_{2} f+\ell_{2}\right),
$$


where the $L_{i}$ are linear differential operators vanishing on $\mathscr{L}$ (commuting with $\left.\mathscr{B}_{m}\right)$ and the $\ell_{i}$ are functions vanishing on $\mathscr{L}(i=1,2)$. Therefore:

$$
\begin{aligned}
& -\left.\mathscr{B}_{-1}\left(\mathscr{B}_{-1}+\frac{\partial}{\partial \alpha}\right) f\right|_{\mathscr{L}} \\
& =-\left.\mathscr{B}_{-1}\left(\frac{\partial f}{\partial u_{1}}+\alpha k_{1}+L_{2} f+\ell_{2}\right)\right|_{\mathscr{L}} \\
& =-\left.\mathscr{B}_{-1}\left(\frac{\partial f}{\partial u_{1}}+L_{2} f\right)\right|_{\mathscr{L}}=-\left.\left(\frac{\partial}{\partial u_{1}}+L_{2}\right) \mathscr{B}_{-1} f\right|_{\mathscr{L}}=-\left.\left(\frac{\partial}{\partial u_{1}}\right) \mathscr{B}_{-1} f\right|_{\mathscr{L}} \\
& =\left.\frac{\partial}{\partial u_{1}}\left(\frac{\partial f}{\partial t_{1}}-\alpha k_{1}+L_{1} f+\ell_{1}\right)\right|_{\mathscr{L}}=\left.\left(\frac{\partial^{2} f}{\partial t_{1} \partial u_{1}}+\frac{\partial}{\partial u_{1}} L_{1} f+\frac{\partial \ell_{1}}{\partial u_{1}}\right)\right|_{\mathscr{L}} \cdot
\end{aligned}
$$

Since

$$
L_{1}=-2 \beta \frac{\partial}{\partial \alpha}-\sum_{i \geq 2}\left(i t_{i} \frac{\partial}{\partial t_{i-1}}+i s_{i} \frac{\partial}{\partial s_{i-1}}+i u_{i} \frac{\partial}{\partial u_{i-1}}\right)
$$

and $\ell_{1}=k_{1} s_{1}+k_{2} u_{1}-\left(k_{1}+k_{2}\right) t_{1}$, one checks that, along the locus $\mathscr{L}$, one has

$$
\frac{\partial}{\partial u_{1}} L_{1} f=0 \quad \text { and } \quad \frac{\partial \ell_{1}}{\partial u_{1}}=k_{2}
$$

yielding the expression for $\frac{\partial^{2} f}{\partial t_{1} \partial u_{1}}$ in 4.5 . A similar procedure applies to establish all the identities above.

\section{A PDE for the Gaussian ensemble with external source}

Consider the Gaussian Hermitian random matrix ensemble $\mathscr{H}_{n}$ with external source $A$, given by the diagonal matrix $(0.16)$ (set $n=k_{1}+k_{2}$ ) and density

$$
\frac{1}{Z_{n}} e^{-\operatorname{Tr}\left(\frac{1}{2} M^{2}-A M\right)} d M
$$

Given a disjoint union of intervals $E:=\bigcup_{i=1}^{r}\left[b_{2 i-1}, b_{2 i}\right] \subset \mathbb{R}$, define the algebra of differential operators, generated by

$$
\mathscr{B}_{k}=\sum_{i=1}^{2 r} b_{i}^{k+1} \frac{\partial}{\partial b_{i}}
$$

Consider the following probability:

$$
\mathbb{P}_{n}(\alpha ; E):=\mathbb{P}(\text { all eigenvalues } \in E)=\frac{1}{Z_{n}} \int_{\mathscr{H}_{n}(E)} e^{-\operatorname{Tr}\left(\frac{1}{2} M^{2}-A M\right)} d M,
$$

where $\mathscr{H}_{n}(E)$ is the set of all Hermitian matrices with all eigenvalues in $E$. The purpose of this section is to prove the following theorem: 
Theorem 5.1. The log of the probability $\mathbb{P}_{n}(a ; E)$ satisfies a fourth-order PDE in $a$ and in the endpoints $b_{1}, \ldots, b_{2 r}$ of the set $E$, with quartic non-linearity:

$$
\begin{aligned}
& \left(F^{+} \mathscr{B}_{-1} G^{-}+F^{-} \mathscr{B}_{-1} G^{+}\right)\left(F^{+} \mathscr{B}_{-1} F^{-}-F^{-} \mathscr{B}_{-1} F^{+}\right) \\
& -\left(F^{+} G^{-}+F^{-} G^{+}\right)\left(F^{+} \mathscr{B}_{-1}^{2} F^{-}-F^{-} \mathscr{B}_{-1}^{2} F^{+}\right)=0,
\end{aligned}
$$

or what is the same

$$
\operatorname{det}\left(\begin{array}{cccc}
G^{+} & \mathscr{B}_{-1} F^{+} & -F^{+} & 0 \\
-G^{-} & \mathscr{B}_{-1} F^{-} & -F^{-} & 0 \\
\mathscr{B}_{-1} G^{+} & \mathscr{B}_{-1}^{2} F^{+} & 0 & -F^{+} \\
-\mathscr{B}_{-1} G^{-} & \mathscr{B}_{-1}^{2} F^{-} & 0 & -F^{-}
\end{array}\right)=0,
$$

where

$$
\begin{aligned}
F^{+}=\mathscr{B}_{-1} \frac{\partial}{\partial \alpha} \log \mathbb{P}_{n}-k_{1}, \quad F^{-}=-\mathscr{B}_{-1}\left(\mathscr{B}_{-1}+\frac{\partial}{\partial \alpha}\right) \log \mathbb{P}_{n}-k_{2} \\
H_{1}^{+}=4 \frac{\partial}{\partial \alpha} \log \mathbb{P}_{n}+4 \alpha k_{1}+\frac{4 k_{1} k_{2}}{\alpha}, \\
H_{1}^{-}=-2\left(\mathscr{B}_{0}-\alpha \frac{\partial}{\partial \alpha}+1\right) \frac{\partial}{\partial \alpha} \log \mathbb{P}_{n}-\frac{4 k_{1} k_{2}}{\alpha} \\
H_{2}^{+}=2\left(\mathscr{B}_{0}-\alpha \frac{\partial}{\partial \alpha}-1-2 \alpha \mathscr{B}_{-1}\right) \frac{\partial}{\partial \alpha} \log \mathbb{P}_{n} \\
H_{2}^{-}=-2\left(\mathscr{B}_{0}-\alpha \frac{\partial}{\partial \alpha}-1\right)\left(\mathscr{B}_{-1}+\frac{\partial}{\partial \alpha}\right) \log \mathbb{P}_{n} \\
2 G^{ \pm}=\left\{H_{1}^{ \pm}, F^{ \pm}\right\}_{\mathscr{B}_{-1}} \mp\left\{H_{2}^{ \pm}, F^{ \pm}\right\}_{\partial / \partial \alpha} .
\end{aligned}
$$

Remark 5.2. It is not surprising that the PDE (5.4) has exactly the same form as the PDE derived in [4] and [5], associated to the Gaussian Unitary Ensemble with an external source, in the case where the source matrix admits two eigenvalues of opposite signs. The only difference is that the expressions for the functions $F^{ \pm}, H_{1}^{ \pm}, H_{2}^{ \pm}$and $G^{ \pm}$obtained here, differ from those in [4]. The reason is that corresponding $\tau$-functions satisfy the same integrable equation (the 3-KP hierarchy, as in Section 3.3), whereas the Virasoro constraints leading to (5.4) are different. In particular, there is no more involution relating the variables $s_{k}$ and $u_{k}$.

Proof of Theorem 5.1 Remember:

$$
\mathbb{P}_{n}(\alpha, E):=\mathbb{P}_{n}(\operatorname{spec} M \subseteq E)=\left.\frac{\tau_{k_{1} k_{2}}(t, s, u ; \alpha, \beta ; E)}{\tau_{k_{1} k_{2}}(t, s, u ; \alpha, \beta ; \mathbb{R})}\right|_{\mathscr{L} \equiv\{t=s=u=\beta=0\}} .
$$

The denominator, that is the integral (3.8) over the whole range, can be expressed in terms of moments, which contain standard Gaussian integrals (the reader is referred 
to the Appendix 1 in [4]), leading to an exact evaluation, with $c_{k_{1} k_{2}}$ a constant, depending on $k_{1}, k_{2}$ only:

$$
\left.\tau_{k_{1}, k_{2}}(t, s, u ; \alpha, \beta ; \mathbb{R})\right|_{\mathscr{L}}=c_{k_{1} k_{2}} \alpha^{k_{1} k_{2}} e^{k_{1}\left(\alpha^{2} / 2\right)} .
$$

Consequently (with $C_{k_{1} k_{2}}$ a constant, depending on $k_{1}, k_{2}$ only):

$$
\log \mathbb{P}_{n}(\alpha, E)=\left.\log \tau_{k_{1}, k_{2}}(t, s, u ; \alpha, \beta ; E)\right|_{\mathscr{L}}-\frac{k_{1}}{2} \alpha^{2}-k_{1} k_{2} \log (\alpha)-C_{k_{1} k_{2}} .
$$

Then, we turn our attention to the numerator $\tau_{k_{1} k_{2}}(t, s, u ; \alpha, \beta ; E)$, more briefly noted as $\tau_{k_{1} k_{2}}$. On the locus $\mathscr{L}$, by (3.9), and (4.4), one finds:

$$
\begin{aligned}
& \frac{\frac{\partial^{2}}{\partial t_{2} \partial s_{1}} \log \tau_{k_{1}, k_{2}}}{\frac{\partial^{2}}{\partial t_{1} \partial s_{1}} \log \tau_{k_{1}, k_{2}}}=\frac{\partial}{\partial t_{1}} \log \left(\frac{\tau_{k_{1}+1, k_{2}}}{\tau_{k_{1}-1, k_{2}}}\right)=-\mathscr{B}_{-1} \log \left(\frac{\tau_{k_{1}+1, k_{2}}}{\tau_{k_{1}-1, k_{2}}}\right)+2 \alpha \\
& \frac{\frac{\partial^{2}}{\partial t_{1} \partial s_{2}} \log \tau_{k_{1}, k_{2}}}{\frac{\partial^{2}}{\partial t_{1} \partial s_{1}} \log \tau_{k_{1}, k_{2}}}=-\frac{\partial}{\partial s_{1}} \log \left(\frac{\tau_{k_{1}+1, k_{2}}}{\tau_{k_{1}-1, k_{2}}}\right)=\frac{\partial}{\partial \alpha} \log \left(\frac{\tau_{k_{1}+1, k_{2}}}{\tau_{k_{1}-1, k_{2}}}\right)
\end{aligned}
$$

In (5.8), acting with $\partial / \partial \alpha$ on the first expression, then acting with $\mathscr{B}_{-1}$ on the second expression, and adding the two, yields:

$$
\frac{\partial}{\partial \alpha}\left(\frac{\frac{\partial^{2}}{\partial t_{2} \partial s_{1}} \log \tau_{k_{1}, k_{2}}}{\frac{\partial^{2}}{\partial t_{1} \partial s_{1}} \log \tau_{k_{1}, k_{2}}}-2 \alpha\right)+\mathscr{B}_{-1}\left(\frac{\frac{\partial^{2}}{\partial t_{1} \partial s_{2}} \log \tau_{k_{1}, k_{2}}}{\frac{\partial^{2}}{\partial t_{1} \partial s_{1}} \log \tau_{k_{1}, k_{2}}}\right)=0 .
$$

This identity can conveniently be expressed as Wronskians:

$$
\left\{\left.\mathscr{B}_{-1} \frac{\partial}{\partial \beta} \log \tau_{k_{1} k_{2}}\right|_{\mathscr{L}}, F^{+}\right\}_{\mathscr{B}_{-1}}=\left\{H_{1}^{+}, \frac{F^{+}}{2}\right\}_{\mathscr{B}_{-1}}-\left\{H_{2}^{+}, \frac{F^{+}}{2}\right\}_{\frac{\partial}{\partial \alpha}}=: G^{+}
$$

where

$$
\begin{aligned}
F^{+} & :=\frac{\partial^{2}}{\partial t_{1} \partial s_{1}} \log \tau_{k_{1}, k_{2}} \\
H_{1}^{+} & :=-2 \frac{\partial^{2}}{\partial t_{1} \partial s_{2}} \log \tau_{k_{1}, k_{2}}+2 \mathscr{B}_{-1} \frac{\partial}{\partial \beta} \log \tau_{k_{1} k_{2}} \\
H_{2}^{+} & :=2\left(\frac{\partial^{2}}{\partial t_{2} \partial s_{1}} \log \tau_{k_{1}, k_{2}}-2 \alpha \frac{\partial^{2}}{\partial t_{1} \partial s_{1}} \log \tau_{k_{1}, k_{2}}\right) .
\end{aligned}
$$


In this way, by virtue of (4.6) and 5.7) one obtains, along the locus $\mathscr{L}$, explicit expressions for $F^{+}, H_{1}^{+}$and $H_{2}^{+}$that are free of partials in $\beta$; namely:

$$
\begin{aligned}
F^{+} & =\mathscr{B}_{-1}\left(\frac{\partial}{\partial \alpha}\right) \log \tau_{k_{1}, k_{2}}-k_{1}=\mathscr{B}_{-1}\left(\frac{\partial}{\partial \alpha}\right) \log \mathbb{P}_{n}-k_{1} \\
H_{1}^{+} & =4\left(\frac{\partial}{\partial \alpha}\right) \log \tau_{k_{1}, k_{2}}=4\left(\frac{\partial}{\partial \alpha}\right) \log \mathbb{P}_{n}+4 \alpha k_{1}+4 \frac{k_{1} k_{2}}{\alpha} \\
H_{2}^{+} & =2\left(\mathscr{B}_{0}-\alpha \frac{\partial}{\partial \alpha}-1-2 \alpha \mathscr{B}_{-1}\right)\left(\frac{\partial}{\partial \alpha}\right) \log \tau_{k_{1}, k_{2}}+4 \alpha k_{1} \\
& =2\left(\mathscr{B}_{0}-\alpha \frac{\partial}{\partial \alpha}-1-2 \alpha \mathscr{B}_{-1}\right)\left(\frac{\partial}{\partial \alpha}\right) \log \mathbb{P}_{n} .
\end{aligned}
$$

Subsequently, one repeats exactly the same operations for the Virasoro and KPidentities involving the $t_{k}$ and $u_{k}$ variables. From (3.10) and (4.4), one finds on $\mathscr{L}$ :

$$
\begin{aligned}
& \frac{\frac{\partial^{2}}{\partial t_{2} \partial u_{1}} \log \tau_{k_{1}, k_{2}}}{\frac{\partial^{2}}{\partial t_{1} \partial u_{1}} \log \tau_{k_{1}, k_{2}}}=\frac{\partial}{\partial t_{1}} \log \left(\frac{\tau_{k_{1}, k_{2}+1}}{\tau_{k_{1}, k_{2}-1}}\right)=-\mathscr{B}_{-1} \log \left(\frac{\tau_{k_{1}, k_{2}+1}}{\tau_{k_{1}, k_{2}-1}}\right) \\
& \frac{\frac{\partial^{2}}{\partial t_{1} \partial u_{2}} \log \tau_{k_{1}, k_{2}}}{\frac{\partial^{2}}{\partial t_{1} \partial u_{1}} \log \tau_{k_{1}, k_{2}}}=-\frac{\partial}{\partial u_{1}} \log \left(\frac{\tau_{k_{1}, k_{2}+1}}{\tau_{k_{1}, k_{2}-1}}\right)=-\left(\mathscr{B}_{-1}+\frac{\partial}{\partial \alpha}\right) \log \left(\frac{\tau_{k_{1}, k_{2}+1}}{\tau_{k_{1}, k_{2}-1}}\right) .
\end{aligned}
$$

So, in 5.12 , acting on the first equation with $\left(\mathscr{B}_{-1}+\frac{\partial}{\partial \alpha}\right)$ and on the second equation with $\mathscr{B}_{-1}$, then subtracting the two yields:

$$
\mathscr{B}_{-1}\left(\frac{\left(\frac{\partial^{2}}{\partial t_{2} \partial u_{1}}-\frac{\partial^{2}}{\partial t_{1} \partial u_{2}}\right) \log \tau_{k_{1}, k_{2}}}{\frac{\partial^{2}}{\partial t_{1} \partial u_{1}} \log \tau_{k_{1}, k_{2}}}\right)+\frac{\partial}{\partial \alpha}\left(\frac{\frac{\partial^{2}}{\partial t_{2} \partial u_{1}} \log \tau_{k_{1}, k_{2}}}{\frac{\partial^{2}}{\partial t_{1} \partial u_{1}} \log \tau_{k_{1}, k_{2}}}\right)=0,
$$

or equivalently (remember the brackets are Wronskians) :

$$
-\left\{\left.\mathscr{B}_{-1} \frac{\partial}{\partial \beta} \log \tau_{k_{1} k_{2}}\right|_{\mathscr{L}}, F^{-}\right\}_{\mathscr{B}_{-1}}=\left\{H_{1}^{-}, \frac{F^{-}}{2}\right\}_{\mathscr{B}_{-1}}+\left\{H_{2}^{-}, \frac{F^{-}}{2}\right\}_{\frac{\partial}{\partial \alpha}}=: G^{-}
$$

in terms of the functions

$$
\begin{aligned}
& F^{-}:=\frac{\partial^{2}}{\partial t_{1} \partial u_{1}} \log \tau_{k_{1}, k_{2}} \\
& H_{1}^{-}:=2\left(\frac{\partial^{2}}{\partial t_{2} \partial u_{1}}-\frac{\partial^{2}}{\partial t_{1} \partial u_{2}}\right) \log \tau_{k_{1}, k_{2}}-2 \mathscr{B}_{-1} \frac{\partial}{\partial \beta} \log \tau_{k_{1} k_{2}} \\
& H_{2}^{-}:=2\left(\frac{\partial^{2}}{\partial t_{2} \partial u_{1}}\right) \log \tau_{k_{1}, k_{2}} .
\end{aligned}
$$


Using the Virasoro constraints (4.5), as well as (5.7), we obtain explicit formulae for $F^{-}, H_{1}^{-}$and $H_{2}^{-}$(which do not contain partials in $\beta$ ):

$$
\begin{aligned}
F^{-} & =-\mathscr{B}_{-1}\left(\mathscr{B}_{-1}+\frac{\partial}{\partial \alpha}\right) \log \tau_{k_{1}, k_{2}}-k_{2} \\
& =-\mathscr{B}_{-1}\left(\mathscr{B}_{-1}+\frac{\partial}{\partial \alpha}\right) \log \mathbb{P}_{n}-k_{2} \\
H_{1}^{-} & =-2\left(\mathscr{B}_{0}-\alpha \frac{\partial}{\partial \alpha}+1\right)\left(\frac{\partial}{\partial \alpha}\right) \log \tau_{k_{1}, k_{2}} \\
& =-2\left(\mathscr{B}_{0}-\alpha \frac{\partial}{\partial \alpha}+1\right)\left(\frac{\partial}{\partial \alpha}\right) \log \mathbb{P}_{n}-4 \frac{k_{1} k_{2}}{\alpha} \\
H_{2}^{-} & =-2\left(\mathscr{B}_{0}-\alpha \frac{\partial}{\partial \alpha}-1\right)\left(\mathscr{B}_{-1}+\frac{\partial}{\partial \alpha}\right) \log \tau_{k_{1}, k_{2}}-4 \alpha k_{1} \\
& =-2\left(\mathscr{B}_{0}-\alpha \frac{\partial}{\partial \alpha}-1\right)\left(\mathscr{B}_{-1}+\frac{\partial}{\partial \alpha}\right) \log \mathbb{P}_{n} .
\end{aligned}
$$

Equations (5.9) and (5.13) form a linear system in

$$
\left.\mathscr{B}_{-1} \frac{\partial \log \tau_{k_{1} k_{2}}}{\partial \beta}\right|_{\mathscr{L}} \quad \text { and }\left.\quad \mathscr{B}_{-1}^{2} \frac{\partial \log \tau_{k_{1} k_{2}}}{\partial \beta}\right|_{\mathscr{L}}
$$

which can be solved to extract the quantities:

$$
\begin{aligned}
& \left.\mathscr{B}_{-1} \frac{\partial \log \tau_{k_{1}, k_{2}}}{\partial \beta}\right|_{\mathscr{L}}=\frac{G^{-} F^{+}+G^{+} F^{-}}{-F^{-}\left(\mathscr{B}_{-1} F^{+}\right)+F^{+}\left(\mathscr{B}_{-1} F^{-}\right)} \\
& \left.\mathscr{B}_{-1}^{2} \frac{\partial \log \tau_{k_{1}, k_{2}}}{\partial \beta}\right|_{\mathscr{L}}=\frac{G^{-}\left(\mathscr{B}_{-1} F^{+}\right)+G^{+}\left(\mathscr{B}_{-1} F^{-}\right)}{-F^{-}\left(\mathscr{B}_{-1} F^{+}\right)+F^{+}\left(\mathscr{B}_{-1} F^{-}\right)} .
\end{aligned}
$$

Finally, subtracting the second relation from $\mathscr{B}_{-1}$ of the first equation, establishes the expected PDE (5.4) and Theorem 5.1. To prove the second equation (5.5), set

$$
X:=\left.\mathscr{B}_{-1} \frac{\partial}{\partial \beta} \log \tau_{k_{1} k_{2}}\right|_{\mathscr{L}} ;
$$

then the matrix in $(5.5)$ annihilates the column $\left(1, X, \mathscr{B}_{-1} X, \mathscr{B}_{-1}^{2} X\right)^{\top}$, and thus the determinant vanishes, concluding the proof of Theorem 5.1 .

\section{A PDE for the transition probability of the $r$-Airy process}

Proof of Theorem 0.4 Remember from section 1 the identity

$$
\begin{aligned}
& \lim _{n \rightarrow \infty} \mathbb{P}_{B r}^{(0, \rho \sqrt{n / 2})}\left(\operatorname{all} x_{i}\left(\frac{1}{1+e^{-2 \tau / n^{1 / 3}}}\right)\right.\left.\in \frac{\left(-\infty, \sqrt{2 n}+\frac{x}{\sqrt{2} n^{1 / 6}}\right)}{2 \cosh \left(\tau / n^{1 / 3}\right)}\right)= \\
& \lim _{n \rightarrow \infty} \mathbb{P}_{n}\left(\rho \sqrt{n} e^{\tau / n^{1 / 3}} ; 2 \sqrt{n}+\frac{(-\infty, x)}{n^{1 / 6}}\right),
\end{aligned}
$$


which for $0 \leq \rho \leq 1$ leads by Theorem 0.1 to a phase transition at $\rho=1$, for which the expression above reduces to $\mathbb{P}\left(\sup \mathscr{A}^{(r)}(\tau) \leq x\right)$, according to 0.7$)$. The above scaling suggests the choice $z=n^{-1 / 6}$ as small parameter and considering the map $(\tau, x) \longmapsto(\alpha, b)$, given by

$$
\alpha=\rho \sqrt{n} e^{\tau / n^{1 / 3}}=\frac{\rho}{z^{3}} e^{\tau z^{2}}, \quad b=2 \sqrt{n}+\frac{x}{n^{1 / 6}}=\frac{2}{z^{3}}+x z
$$

with inverse map $(\alpha, b) \longmapsto(\tau, x)$, given by

$$
\tau=\frac{1}{z^{2}} \log \left(\frac{\alpha z^{3}}{\rho}\right), x=\frac{b}{z}-\frac{2}{z^{4}} .
$$

Setting

$$
\begin{aligned}
\tilde{Q}(\tau, x) & :=\log \mathbb{P}_{n}\left(\rho \sqrt{n} e^{\tau / n^{1 / 3}} ; 2 \sqrt{n}+\frac{(-\infty, x)}{n^{1 / 6}}\right) \\
& =\log \mathbb{P}_{n}\left(\frac{\rho}{z^{3}} e^{\tau z^{2}} ; \frac{2}{z^{3}}+(-\infty, x) z\right)
\end{aligned}
$$

yields, via the inverse map,

$$
\tilde{Q}\left(\frac{1}{z^{2}} \log \left(\frac{\alpha z^{3}}{\rho}\right), \frac{b}{z}-\frac{2}{z^{4}}\right)=\log \mathbb{P}_{n}(\alpha,(-\infty, b)) .
$$

From Corollary 2.4 (section 2) it follows that for $z \rightarrow 0$,

$$
\tilde{Q}(\tau, x)=Q(\tau, x)+O\left(z^{2}\right),
$$

with $Q(\tau, x)$ independent of $z$.

Since we have shown that $\log \mathbb{P}_{n}(\alpha,(-\infty, b))$ satisfies the PDE (5.4) of Theorem 5.1. with $\mathscr{B}_{-1} \equiv \frac{\partial}{\partial b}$ and $\mathscr{B}_{0} \equiv b \frac{\partial}{\partial b}$, it follows that, to get a PDE for the limiting case, we just need to estimate:

$$
\left\{\begin{array}{ll}
\left(F^{+} \mathscr{B}_{-1} G^{-}+F^{-} \mathscr{B}_{-1} G^{+}\right)\left(F^{+} \mathscr{B}_{-1} F^{-}-F^{-} \mathscr{B}_{-1} F^{+}\right) \\
-\left(F^{+} G^{-}+F^{-} G^{+}\right)\left(F^{+} \mathscr{B}_{-1}^{2} F^{-}-F^{-} \mathscr{B}_{-1}^{2} F^{+}\right)
\end{array}\right\} \mid \begin{aligned}
& \alpha \mapsto\left(\rho / z^{3}\right) e^{\tau z^{2}} \\
& b \mapsto x z+\frac{2}{z^{3}} \\
& n \mapsto \frac{1}{z^{6}}
\end{aligned}
$$

To do this, the various expressions in the bracket must be computed in terms of the function $\tilde{Q}(\tau, x)$. By 6.1 and 6.2 , one immediately gets:

$$
\frac{\partial \log \mathbb{P}_{n}}{\partial \alpha}=\frac{1}{\alpha z^{2}}\left(\frac{\partial \tilde{Q}}{\partial \tau}\right)=\frac{z}{\rho} e^{-\tau z^{2}}\left(\frac{\partial \tilde{Q}}{\partial \tau}\right), \quad \frac{\partial \log \mathbb{P}_{n}}{\partial b}=\frac{1}{z}\left(\frac{\partial \tilde{Q}}{\partial x}\right) .
$$

Hereafter, to shorten notation, we will write the partials as

$$
\tilde{Q}_{\tau x} \equiv \frac{\partial^{2}}{\partial \tau \partial x} \tilde{Q}, \tilde{Q}_{\tau \tau x} \equiv \frac{\partial^{3}}{\partial \tau^{2} \partial x} \tilde{Q}, \text { etc. }
$$


Without taking a limit yet, but expanding asymptotically the expressions in powers of $z$, we find

$$
\begin{aligned}
\left(F^{+} \mathscr{B}_{-1} F^{-}-F^{-} \mathscr{B}_{-1} F^{+}\right) & =\frac{\tilde{Q}_{\tau x x}}{\rho z^{7}}-\frac{\tau \tilde{Q}_{\tau x x}}{\rho z^{5}}+\frac{\tilde{Q}_{x x x}\left(r \rho-\tilde{Q}_{\tau x}\right)+\tilde{Q}_{\tau x x}\left(\tilde{Q}_{x x}+\frac{\tau^{2}}{2}\right)}{\rho z^{3}}+O\left(\frac{1}{z}\right) \\
\left(F^{+} \mathscr{B}_{-1}^{2} F^{-}-F^{-} \mathscr{B}_{-1}^{2} F^{+}\right) & =\frac{\tilde{Q}_{\tau x x x}}{\rho z^{8}}-\frac{\tau \tilde{Q}_{\tau x x x}}{\rho z^{6}}+\frac{\tilde{Q}_{x x x x}\left(r \rho-\tilde{Q}_{\tau x}\right)+\tilde{Q}_{\tau x x x}\left(\tilde{Q}_{x x}+\frac{\tau^{2}}{2}\right)}{\rho z^{4}}+O\left(\frac{1}{z^{2}}\right) .
\end{aligned}
$$

In order to compute the expansions of $G^{ \pm}$(which are respectively defined in $(5.9)$ and (5.13) and of $\mathscr{B}_{-1} G^{ \pm}$, we need the asymptotics of $H_{i}^{ \pm}$, as defined in $(5.11)$ and (5.15). In the end, one finds

$$
\begin{aligned}
& F^{+} G^{-}+F^{-} G^{+}=\frac{2 \tilde{Q}_{\tau x x}}{\rho^{2}}\left[2 \tilde{Q}_{\tau x}+r(\rho-1)^{2}\right] \frac{1}{z^{10}} \\
& -\frac{2}{\rho^{3} z^{8}}\left(\begin{array}{c}
\tilde{Q}_{\tau x}\left((\rho-1) \tilde{Q}_{\tau \tau x}+4 \rho \tau \tilde{Q}_{\tau x x}\right) \\
-2 r \rho(\rho-1)\left(\tilde{Q}_{\tau \tau x}+\tau \tilde{Q}_{\tau x x}\right)
\end{array}\right)+\frac{\mathscr{T}}{z^{6}}+O\left(\frac{1}{z^{4}}\right) \\
& F^{+} \mathscr{B}_{-1} G^{-}+F^{-} \mathscr{B}_{-1} G^{+}=\frac{2 \tilde{Q}_{\tau x x x}}{\rho^{2}}\left[2 \tilde{Q}_{\tau x}+r(1-\rho)^{2}\right] \frac{1}{z^{11}} \\
& -\frac{2}{\rho^{3} z^{9}}\left(\begin{array}{c}
\tilde{Q}_{\tau x}\left((\rho-1) \tilde{Q}_{\tau \tau x x}+4 \rho \tau \tilde{Q}_{\tau x x x}\right) \\
-2 r \rho(\rho-1)\left(\tilde{Q}_{\tau \tau x x}+\tau \tilde{Q}_{\tau x x x}\right)
\end{array}\right)+\frac{\mathscr{T}^{\prime}}{z^{7}}+O\left(\frac{1}{z^{5}}\right),
\end{aligned}
$$

where $\mathscr{T}$ and $\mathscr{T}^{\prime}$ are given by the following expressions and where $\mathscr{T}_{1}$ and $\mathscr{T}_{1}^{\prime}$ denote further expressions in the derivatives of $\tilde{Q}$,

$$
\begin{aligned}
\mathscr{T}= & 2 r^{2} \tilde{Q}_{x x x}+2 r\left(\tilde{Q}_{\tau x x}\left(\tilde{Q}_{x x}+\tau^{2}-x\right)+2 \tau \tilde{Q}_{\tau \tau x}+2 \tilde{Q}_{\tau x}+\tilde{Q}_{\tau \tau \tau}\right)-2 \tilde{Q}_{\tau x}^{2}\left(\tilde{Q}_{x x x}+2\right) \\
& -2 \tilde{Q}_{\tau x}\left(\tau \tilde{Q}_{\tau \tau x}+\tilde{Q}_{\tau \tau \tau}-\tilde{Q}_{\tau x x}\left(\tilde{Q}_{x x}+4 \tau^{2}+x\right)\right)+2 \tilde{Q}_{\tau x x} \tilde{Q}_{\tau}+\tilde{Q}_{\tau \tau} \tilde{Q}_{\tau \tau x}+(\rho-1) \mathscr{T}_{1}, \\
\mathscr{T}^{\prime}= & 2 r^{2} \tilde{Q}_{x x x x}+2 r\left(\tilde{Q}_{\tau x x x}\left(\tilde{Q}_{x x}+\tau^{2}-x\right)+2 \tau \tilde{Q}_{\tau \tau x x}+\tilde{Q}_{\tau x x}+\tilde{Q}_{\tau \tau \tau x}\right) \\
& -2 \tilde{Q}_{\tau x}^{2} \tilde{Q}_{x x x x}-2 \tilde{Q}_{\tau x}\left(\tau \tilde{Q}_{\tau \tau x x}+\tilde{Q}_{\tau \tau \tau x}+2 \tilde{Q}_{\tau x x}-\tilde{Q}_{\tau x x x}\left(\tilde{Q}_{x x}+4 \tau^{2}+x\right)\right) \\
& +\tilde{Q}_{\tau \tau x}^{2}+\tilde{Q}_{\tau \tau} \tilde{Q}_{\tau \tau x x}-\tilde{Q}_{\tau x x} \tilde{Q}_{\tau \tau \tau}+2 \tilde{Q}_{\tau x x x} \tilde{Q}_{\tau}+(\rho-1) \mathscr{T}_{1}^{\prime} .
\end{aligned}
$$

Consequently, using the two leading orders for the expressions above, one obtains for small $z$ :

$$
\begin{aligned}
0 & =\left\{\begin{array}{l}
\left(F^{+} \mathscr{B}_{-1} G^{-}+F^{-} \mathscr{B}_{-1} G^{+}\right)\left(F^{+} \mathscr{B}_{-1} F^{-}-F^{-} \mathscr{B}_{-1} F^{+}\right) \\
-\left(F^{+} G^{-}+F^{-} G^{+}\right)\left(F^{+} \mathscr{B}_{-1}^{2} F^{-}-F^{-} \mathscr{B}_{-1}^{2} F^{+}\right)
\end{array}\right\} \mid \begin{array}{l}
\alpha \mapsto\left(\rho / z^{3}\right) e^{\tau z^{2}} \\
\begin{array}{l} 
\\
b \\
n \mapsto \frac{1}{z^{6}}
\end{array}
\end{array} \\
= & \frac{4(\rho-1)}{\rho^{4} z^{16}}\left(2 r \rho-\frac{\partial^{2} \tilde{Q}}{\partial \tau \partial x}\right)\left\{\frac{\partial^{3} \tilde{Q}}{\partial \tau^{2} \partial x}, \frac{\partial^{3} \tilde{Q}}{\partial \tau \partial x^{2}}\right\}_{x}+\frac{\mathscr{E}(\tilde{Q})+(\rho-1) \mathscr{F}(\tilde{Q})}{z^{14}}+O\left(\frac{1}{z^{12}}\right),
\end{aligned}
$$


where $\mathscr{E}(\tilde{Q})$ is given by

$$
\begin{aligned}
\mathscr{E}(\tilde{Q})= & 2\left(r-\frac{\partial^{2} \tilde{Q}}{\partial \tau \partial x}\right)^{2}\left\{\frac{\partial^{3} \tilde{Q}}{\partial \tau \partial x^{2}}, \frac{\partial^{3} \tilde{Q}}{\partial x^{3}}\right\}_{x} \\
& +2\left(r-\frac{\partial^{2} \tilde{Q}}{\partial \tau \partial x}\right)\left\{\frac{\partial^{3} \tilde{Q}}{\partial \tau \partial x^{2}}, \frac{\partial}{\partial \tau}\left(\frac{\partial}{\partial \tau}\left(\frac{\partial \tilde{Q}}{\partial \tau}+\tau \frac{\partial \tilde{Q}}{\partial x}\right)-x \frac{\partial^{2} \tilde{Q}}{\partial x^{2}}\right)\right\}_{x} \\
(6.6) \quad & +\left\{\frac{\partial^{3} \tilde{Q}}{\partial \tau \partial x^{2}}, \frac{\partial^{3} \tilde{Q}}{\partial \tau^{2} \partial x}\left(2 r \tau+\frac{\partial^{2} \tilde{Q}}{\partial \tau^{2}}\right)\right\}_{x}+\left(\frac{\partial^{3} \tilde{Q}}{\partial \tau \partial x^{2}}\right)^{2} \frac{\partial}{\partial \tau}\left(\frac{\partial^{2} \tilde{Q}}{\partial \tau^{2}}+2 \frac{\partial \tilde{Q}}{\partial x}\right),
\end{aligned}
$$

where $\mathscr{F}(\tilde{Q})$ is a similar expression, that will not be needed, and where the bracket is a Wronskian associated to the "space" operator $\partial / \partial x$.

Hence, for $0 \leq \rho<1$, taking the limit $z \rightarrow 0$ above and using $(6.3$, yields the equation

$$
\left(2 r \rho-\frac{\partial^{2} Q}{\partial \tau \partial x}\right)\left\{\frac{\partial^{3} Q}{\partial \tau^{2} \partial x}, \frac{\partial^{3} Q}{\partial \tau \partial x^{2}}\right\}_{x}=0
$$

which is trivially satisfied; indeed, from other considerations we know that $e^{Q}$ is the Tracy-Widom distribution, which of course is $\tau$-independent.

However, in the critical case $\rho=1$, the leading term has order $1 / z^{14}$, with coefficient $\mathscr{E}(\tilde{Q}(\tau, x ; z))$, as in $(6.6)$. Then taking a limit in 6.5 when $z \rightarrow 0$ and using 6.3 , forces upon us the equation

$$
\lim _{z \rightarrow 0} \mathscr{E}(\tilde{Q}(\tau, x ; z))=\mathscr{E}(Q(\tau, x))=0,
$$

which an easy computation shows can be written as

$$
\begin{aligned}
& \left\{\frac{\partial^{3} Q}{\partial \tau \partial x^{2}},\left[\begin{array}{c}
r^{2} \frac{\partial^{3} Q}{\partial x^{3}}+r\left(\frac{\partial^{2}}{\partial \tau^{2}}\left(\frac{\partial Q}{\partial \tau}+2 \tau \frac{\partial Q}{\partial x}\right)-\frac{\partial^{3}(x Q)}{\partial \tau \partial x^{2}}+2\left\{\frac{\partial^{2} Q}{\partial \tau \partial x}, \frac{\partial^{2} Q}{\partial x^{2}}\right\}_{x}\right) \\
+\frac{1}{2} \frac{\partial^{3} Q}{\partial \tau^{2} \partial x} \frac{\partial^{2} Q}{\partial \tau^{2}}-\frac{\partial^{2} Q}{\partial \tau \partial x} \frac{\partial^{3} Q}{\partial \tau^{3}}+\left(\frac{\partial^{2} Q}{\partial \tau \partial x}\right)^{2} \frac{\partial^{3} Q}{\partial x^{3}}+\left\{\frac{\partial^{2} Q}{\partial \tau \partial x}, \frac{\partial(\tau Q)}{\partial \tau^{2}}\right\}_{x}
\end{array}\right]_{x}\right. \\
& -\frac{1}{2}\left(\frac{\partial^{3} Q}{\partial \tau \partial x^{2}}\right)^{2}\left(\frac{\partial^{3} Q}{\partial \tau^{3}}-4 \frac{\partial^{2} Q}{\partial \tau \partial x} \frac{\partial^{3} Q}{\partial x^{3}}\right)=0
\end{aligned}
$$

and further rewritten as equation (0.12), ending the proof of Theorem 0.4.

Remark 6.1. This "phase transition" at $\rho=1$ is completely analogous to the results found in [8] and [29] for small rank perturbations of random Hermitian matrices.

\section{Remote past asymptotics}

The aim of this section is to study the behavior of the $r$-Airy process $\mathscr{A}^{(r)}(t)$ for $t \rightarrow-\infty$, as stated in Theorem 0.7 . In this section $\tau$ will be systematically replaced by $t$. This theorem will be rephrased as Theorem 7.1, which includes some additional details. 
Theorem 7.1. The log of the probability of the $r$-Airy process $Q(t, x)=$ $\log \mathbb{P}\left(\sup \mathscr{A}^{(r)}(t) \leq x\right)$ admits an asymptotic expansion, as $t \rightarrow-\infty$, having the following form

$$
Q(t, x)=Q_{0}(x)+\frac{1}{t} Q_{1}(x)+\frac{1}{t^{2}} Q_{2}(x)+\ldots
$$

for the initial condition

$$
\lim _{t \rightarrow-\infty} Q(t, x):=Q_{0}(x):=\log \mathbb{P}(\sup \mathscr{A}(t) \leq x)=-\int_{x}^{\infty}(\alpha-x) g^{2}(\alpha) d \alpha,
$$

and where

$$
\begin{aligned}
Q_{1} & =r Q_{0}^{\prime}, \quad Q_{2}=\frac{r^{2}}{2 !} Q_{0}^{\prime \prime}, \quad Q_{3}=\frac{r^{3}}{3 !} Q_{0}^{\prime \prime \prime}+\frac{r}{3} x Q_{0}^{\prime}, \quad Q_{4}=\frac{r^{4}}{4 !} Q_{0}^{i v}+\frac{r^{2}}{3} x Q_{0}^{\prime \prime}+\frac{7 r^{2}}{12} Q_{0}^{\prime}, \\
Q_{5} & =\frac{r^{5}}{5 !} Q_{0}^{v}+\frac{r^{3}}{32 !} x Q_{0}^{\prime \prime \prime}+\frac{7 r^{3}}{12} Q_{0}^{\prime \prime}+\frac{r}{5} \mathscr{F}_{5} \\
Q_{6} & =\frac{r^{6}}{6 !} Q_{0}^{v i}+\frac{r^{4}}{33 !} \times Q_{0}^{i v}+\frac{7 r^{4}}{122 !} Q_{0}^{\prime \prime \prime}+\frac{r^{2}}{5}\left(\mathscr{F}_{5}^{\prime}+\frac{5}{18}\left(x^{2} Q_{0}^{\prime \prime}+13\left(x+c_{6}\right) Q_{0}^{\prime}\right)\right) \\
\vdots & \\
Q_{n} & =\frac{r^{n}}{n !} Q_{0}^{(n)}+\frac{r^{n-2} x}{3(n-3) !} Q_{0}^{(n-2)}+\frac{7 r^{n-2}}{12(n-4) !} Q_{0}^{(n-3)}+\sum_{i=2}^{\left[\frac{n-1}{2}\right]} r^{n-2 i} Q_{n, n-2 i}(x)
\end{aligned}
$$

for some constant $c_{6}$ and with

$$
\mathscr{F}_{5}:=x^{2} Q_{0}^{\prime}+4 x Q_{0}+Q_{0}^{\prime 2}+10 \int_{x}^{\infty} Q_{0}-6 \int_{x}^{\infty} d y \int_{y}^{\infty} d u Q_{0}^{\prime \prime 2} .
$$

Also

$$
\begin{aligned}
\mathbb{P}\left(\sup \mathscr{A}^{(r)}(t) \leq x\right)= & \mathbb{P}\left(\sup \mathscr{A}(t) \leq\left(x+\frac{r}{t}\right)\left(1+\frac{r}{3 t^{3}}\right)+\frac{r^{2}}{4 t^{4}}\right) \\
& \times\left(1+\frac{r \mathscr{F}_{5}}{5 t^{5}}+O\left(\frac{1}{t^{6}}\right)\right) .
\end{aligned}
$$

The mean and variance of the right edge of the process behave as

$$
\begin{aligned}
\mathbb{E}\left(\sup \mathscr{A}^{(r)}(t)\right) & =\mathbb{E}\left(\sup \mathscr{A}^{(0)}(t)\right)\left(1-\frac{r}{3 t^{3}}\right)-\frac{r}{t}-\frac{r^{2}}{4 t^{4}}+O\left(\frac{1}{t^{5}}\right) \\
\operatorname{var}\left(\sup \mathscr{A}^{(r)}(t)\right) & =\operatorname{var}\left(\sup \mathscr{A}^{(0)}(t)\right)\left(1-\frac{2 r}{3 t^{3}}\right)+O\left(\frac{1}{t^{5}}\right) .
\end{aligned}
$$

Remember the $r$-Airy kernel

$$
K_{t}^{(r)}(u, v)=\int_{0}^{\infty} d w A_{r}^{-}(u+w ; t) A_{r}^{+}(v+w ; t),
$$

as in $(0.9)$, where $A_{r}^{ \pm}(u ; \tau)$ is given by $(0.8)$, where $C$ is a contour running from $\infty e^{5 i \pi / 6}$ to $\infty e^{i \pi / 6}$, such that $-i t$ lies above the contour. In this section one lets $t \rightarrow$ $-\infty$, which, of course, implies that $-i t$ will remain above the contour $C$ and thus this limit is compatible with the contour just mentioned. Letting $t \rightarrow+\infty$ would 
require a drastic change of the functions $A_{r}^{ \pm}$. In this section the subscript $t$ will occasionally be omitted from the $r$-Airy kernel $K_{t}^{(r)}(u, v)$. Note that $K_{t}^{(0)}(u, v)=$ $K^{(0)}(u, v)$ is the Airy kernel, which is independent of $t$,

$$
K^{(0)}(u, v):=\frac{A(u) A^{\prime}(v)-A^{\prime}(u) A(v)}{u-v}=\int_{0}^{\infty} A(w+u) A(w+v) d w,
$$

where

$$
A(u)=A_{0}^{ \pm}(u)=\int_{C} e^{\frac{1}{3} i a^{3}+i a u} \frac{d a}{2 \pi}
$$

is the Airy function, satisfying the ordinary differential equation $A^{\prime \prime}(u)=u A(u)$ and behaving asymptotically as

$$
A(x)=\frac{e^{-\frac{2}{3} x^{3 / 2}}}{2 \sqrt{\pi} x^{1 / 4}}\left(1+\sum_{1}^{\infty} \frac{\alpha_{i}}{\left(x^{3 / 2}\right)^{i}}+\ldots\right), \text { as } x \rightarrow \infty .
$$

The ODE and $\lim _{x \rightarrow \infty} A(x)=0$ imply the following formulae, upon differentiation by $x$,

$$
\begin{aligned}
& \int_{x}^{\infty} A^{2}(u) d u=\left(A^{\prime 2}-A A^{\prime \prime}\right)(x) \\
& \int_{x}^{\infty} A^{\prime 2}(u) d u=-\frac{1}{3}\left(\left(A^{2}\right)^{\prime}+x\left(A^{\prime 2}-A A^{\prime \prime}\right)\right)(x) .
\end{aligned}
$$

Also remember the Tracy-Widom distribution [26]

$$
\operatorname{det}\left(I-K^{(0)}\right)_{x}=\exp \left(-\int_{x}^{\infty}(\alpha-x) g^{2}(\alpha) d \alpha\right),
$$

where $g(\alpha)$ is the Hastings-MacLeod solution 0.14 of Painlevé II. The following shorthand notation will be used for integers $\ell \geq 1$,

$$
O\left(A^{\ell}\right)=O\left(\left(\frac{e^{-\frac{2}{3} x^{\frac{3}{2}}}}{2 \sqrt{\pi} x^{1 / 4}}\right)^{\ell} x^{k}\right), \text { for } x \rightarrow \infty,
$$

whatever be the power $k \in \mathbb{R}$.

\section{Lemma 7.2. Given}

$$
Q_{0}(x)=\log \operatorname{det}\left(I-K^{(0)}\right)_{x}=-\int_{x}^{\infty}(\alpha-x) g^{2}(\alpha) d \alpha,
$$

one checks

$$
\begin{aligned}
Q_{0}^{\prime}(x) & =\int_{x}^{\infty} g^{2}(u) d u=A^{\prime 2}(x)-A^{\prime \prime}(x) A(x)+O\left(A^{3}\right) \\
Q_{0}^{(n)}(x) & =-\left(g^{2}\right)^{(n-2)}(x)=-\left(A^{2}\right)^{(n-2)}(x)+O\left(A^{3}\right), \text { for } n \geq 2 .
\end{aligned}
$$


Proof. The estimates follow from (7.3) and an improved version of the estimate (0.14) by Hastings-McLeod [18], namely:

$$
g(x)=\mathrm{A}(x)+O\left(\frac{e^{-\frac{4}{3} x^{3 / 2}}}{x^{1 / 4}}\right) \text { for } x \nearrow \infty,
$$

which is to be interpreted as a genuine asymptotic formula; i.e., it can be both integrated and differentiated.

For future use, one needs the following estimates for the Airy function:

Lemma 7.3. For $x \rightarrow \infty$, one has the estimates

$$
\begin{aligned}
& \int_{x}^{\infty} A(u) d u=\frac{e^{-\frac{2}{3} x^{3 / 2}}}{2 \sqrt{\pi} x^{3 / 4}}\left(1+\sum_{1}^{\infty} \frac{c_{i}^{\prime}}{\left(x^{3 / 2}\right)^{i}}\right)=O(A) \\
& \int_{x}^{\infty} A^{2}(u) d u=\left(A^{\prime 2}-A^{\prime \prime} A\right)(x)=\frac{e^{-\frac{4}{3} x^{3 / 2}}}{8 \pi x}\left(1+\sum_{1}^{\infty} \frac{c_{i}^{\prime \prime}}{\left(x^{3 / 2}\right)^{i}}\right)=O\left(A^{2}\right) .
\end{aligned}
$$

Proof. Upon using integration by parts and upon substituting the asymptotic formula (7.2) for the Airy function, one computes for instance,

$$
\begin{aligned}
\int_{x}^{\infty} A^{2}(u) d u & =\int_{x}^{\infty} \frac{-1}{8 \pi u}\left(1+\sum_{1}^{\infty} \frac{\tilde{c}_{i}^{\prime}}{\left(u^{3 / 2}\right)^{i}}\right) d\left(e^{-\frac{4}{3} u^{3 / 2}}\right) \\
& =\frac{e^{-\frac{4}{3} x^{3 / 2}}}{8 \pi x}\left(1+\sum_{1}^{\infty} \frac{\tilde{c}_{i}^{\prime}}{\left(x^{3 / 2}\right)^{i}}\right)-\frac{1}{8 \pi} \int_{x}^{\infty} \frac{e^{-\frac{4}{3} u^{3 / 2}}}{u^{2}}\left(1+\sum_{1}^{\infty} \frac{\tilde{c}_{i}^{\prime \prime}}{\left(u^{3 / 2}\right)^{i}}\right) .
\end{aligned}
$$

Further terms in the expansion can be obtained by differentiation by parts and similarly for the first expression, thus ending the proof of Lemma 7.3.

Given a kernel $F(y, u)$ acting on $L^{2}(E)$ with $E \subset \mathbb{R}$ and a bounded continuous real function $f$ on $E$, define the norms

$$
\|F\|_{1}=\sup _{y \in E} \int_{E}|F(y, u)| d u \text { and }\|f\|_{\infty}=\sup _{y \in E}|f(y)| .
$$

If $f$ is a function of several variables, the sup is taken over all variables. Then

$$
\begin{aligned}
\|F G\|_{1} & \leq \sup _{y \in E} \int_{E} d z \int_{E} d u|F(y, u) G(u, z)| \\
& \leq \sup _{y \in E} \int_{E} d u|F(y, u)| \sup _{u \in E} \int_{E} d z|G(u, z)|=|| F\left\|_{1}\right\| G \|_{1}
\end{aligned}
$$

Hence 12

$$
\left\|\sum_{1}^{\infty} F^{n}\right\|_{1} \leq \sum_{1}^{\infty}\|F\|_{1}^{n}=\frac{\|F\|_{1}}{1-\|F\|_{1}}
$$

12 Obviously the estimate below requires $\|F\|_{1}<1$. In the application, this is achieved by restricting the domain of the operator to the interval $(x, \infty)$ for sufficiently large $x$. 
and

$$
\|F f\|_{\infty}=\sup _{y \in E} \int_{E}|F(y, u) f(u)| d u \leq \sup _{u \in E}|f(u)| \sup _{y \in E} \int_{E}|F(y, u)| d u=\|F\|_{1}\|f\|_{\infty} .
$$

Given the kernel $K^{(0)}$, define the resolvent kernel $R$ by

$$
I+R:=\left(I-K^{(0)}\right)^{-1}
$$

Then readily

$$
R-K^{(0)}=K^{(0) 2}\left(I+K^{(0)}+K^{(0) 2}+\ldots\right)=\left(I-K^{(0)}\right)^{-1} K^{(0) 2} .
$$

Lemma 7.4. One has the following estimates

$$
\left\|K^{(0)}\right\|_{1}=O\left(A^{2}\right),\left\|K^{(0)}\right\|_{\infty}=O\left(A^{2}\right), \quad \text { and } \quad\left\|R-K^{(0)}\right\|_{\infty}=O\left(A^{4}\right) .
$$

Proof. Using the fact that $A(u) \geq 0$ is monotonically decreasing for $u \geq 0$, and setting $E=(x, \infty)$,

$$
\begin{aligned}
\left\|K^{(0)}\right\|_{1} & =\sup _{u \in(x, \infty)} \int_{x}^{\infty} d v\left|\int_{0}^{\infty} d w A(u+w) A(v+w)\right| \\
& \leq \sup _{u \in(x, \infty)} \int_{x}^{\infty} d v A(v) \int_{0}^{\infty} d w A(u+w) \\
& \leq\left(\int_{x}^{\infty} d v A(v)\right)^{2}=O\left(A^{2}\right)
\end{aligned}
$$

by Lemma 7.3, while

$$
\begin{aligned}
\left\|K^{(0)}\right\|_{\infty}=\sup _{u, v \in(x, \infty)}|K(u, v)| & =\sup _{u, v \in(x, \infty)}\left|\int_{0}^{\infty} A(u+w) A(v+w) d w\right| \\
& \leq \int_{0}^{\infty} A(x+w)^{2} d w \\
& =\int_{x}^{\infty} A(w)^{2} d w=O\left(A^{2}\right),
\end{aligned}
$$


also by Lemma 7.3, Considering the function $R(\cdot, v)$ for fixed $v$, one has, using (7.5),

$$
\begin{aligned}
\left\|R(\cdot, v)-K^{(0)}(\cdot, v)\right\|_{\infty} & =\left\|\left(I-K^{(0)}\right)^{-1}\left(K^{(0) 2}(\cdot, v)\right)\right\|_{\infty} \\
& \leq\left\|\left(I-K^{(0)}\right)^{-1}\right\|_{1}\left\|K^{(0) 2}(\cdot, v)\right\|_{\infty} \\
& \leq \frac{1}{1-\left\|K^{(0)}\right\|_{1}}\left\|K^{(0) 2}(\cdot, v)\right\|_{\infty} \\
& \leq \frac{1}{1-\left\|K^{(0)}\right\|_{1}}\left\|K^{(0)}\right\|_{1}\left\|K^{(0)}(\cdot, v)\right\|_{\infty} .
\end{aligned}
$$

Hence, with $\|K\|_{\infty}:=\sup _{u, v \in E}|K(u, v)|$,

$$
\left\|R-K^{(0)}\right\|_{\infty}:=\sup _{u, v \in E}\left|R(u, v)-K^{(0)}(u, v)\right| \leq \frac{\left\|K^{(0)}\right\|_{1}}{1-\left\|K^{(0)}\right\|_{1}}\left\|K^{(0)}\right\|_{\infty}=O\left(A^{4}\right),
$$

ending the proof of Lemma 7.4 .

The next point is to get an asymptotic expansion for the Fredholm determinant $\operatorname{det}\left(I-K_{t}^{(r)}\right)_{x, \infty}$ in $t$, using the asymptotic expansion of the kernel $K_{t}^{(r)}(u, v)$ in $t$ (Lemma 2.2); this leads to the next Lemma:

Lemma 7.5. The following probability for the r-Airy process has an asymptotic expansion in $1 / t$ of the following form

$$
Q(t, x)=\log \mathbb{P}\left(\sup \mathscr{A}^{(r)}(t) \leq x\right)=\log \operatorname{det}\left(I-K_{t}^{(r)}\right)_{x}=Q_{0}(x)+\sum_{n=1}^{\infty} \frac{Q_{n}(x)}{t^{n}}
$$

where

$$
Q_{n}(x)=\sum_{i=0}^{\left[\frac{n-1}{2}\right]} r^{n-2 i} Q_{n, n-2 i}(x)=-\operatorname{Tr} K_{n}^{(r)}+O\left(A^{4}\right)
$$


where $Q_{0}=\log \mathbb{P}(\sup \mathscr{A}(t) \leq x)$ and where the $Q_{i} \rightarrow 0$ and have all their derivatives $\rightarrow 0$ for $x \rightarrow \infty$. Moreover, for $x \rightarrow \infty$, one has

$$
\begin{aligned}
Q_{1}(x)= & r\left(A^{\prime 2}-A A^{\prime \prime}+O\left(A^{4}\right)\right) \\
Q_{2}(x)= & -\frac{r^{2}}{2}\left(A^{2}+O\left(A^{4}\right)\right) \\
Q_{3}(x)= & -\frac{r^{3}}{3 !}\left(\left(A^{2}\right)^{\prime}+O\left(A^{4}\right)\right)-\frac{r}{3}\left(\left(A^{2}\right)^{\prime}+3 \int_{x}^{\infty} A^{\prime 2}(u) d u+O\left(A^{4}\right)\right)+O\left(A^{4}\right) \\
\vdots & \\
Q_{n}(x)=- & \frac{r^{n}}{n !}\left(\left(A^{2}\right)^{(n-2)}+O\left(A^{4}\right)\right) \\
& -\frac{r^{n-2}}{(n-3) !}\left(\frac{3 n-1}{24}\left(A^{2}\right)^{\prime \prime}-\frac{n-1}{2}\left(A^{\prime}\right)^{2}+O\left(A^{4}\right)\right)^{(n-4)} \\
& +\sum_{i=2}^{n-1} r^{n-2 i} T_{n, n-2 i}(A)+O\left(A^{4}\right),
\end{aligned}
$$

with $T_{n, n-2 i}(A)=$ quadratic polynomial of $A$ and $A^{\prime}$, with coefficients depending on $x+\int_{x}^{\infty}$ (quadratic polynomial of $A$ and $A^{\prime}$, with coefficients depending on $x$ ).

Proof. We shall always operate in $L^{2}(x, \infty)$, so that occasionally the $x$ will be suppressed. Then, using the asymptotics for the kernel $K_{t}^{(r)}$ as in Lemma 2.2, one has the following:

$$
I-K_{t}^{(r)}=I-K^{(0)}-\frac{K_{1}^{(r)}}{t}-\frac{K_{2}^{(r)}}{t^{2}}-\ldots=\left(I-K^{(0)}\right)\left(I-\sum_{i \geq 1} \frac{L_{i}}{t^{i}}\right)
$$

with (the resolvent operator $R$ of the Airy kernel is defined in (7.4))

$$
L_{i}=\left(I-K^{(0)}\right)^{-1} K_{i}^{(r)}=(I+R) K_{i}^{(r)} .
$$

Using $\log (1-z)=-z-\frac{z^{2}}{2}-\frac{z^{3}}{3}-\ldots$, one finds

$$
Q(t, x)=\log \operatorname{det}\left(I-K_{t}^{(r)}\right)_{x}=\operatorname{Tr} \log \left(I-K_{t}^{(r)}\right)=\operatorname{Tr} \log \left(I-K^{(0)}\right)+\sum_{i=1}^{\infty} \frac{Q_{i}}{t^{i}},
$$

where

$$
\begin{aligned}
& Q_{1}=-\operatorname{Tr} L_{1}, \quad Q_{2}=-\operatorname{Tr}\left(L_{2}+\frac{L_{1}^{2}}{2}\right), \quad Q_{3}=-\operatorname{Tr}\left(L_{3}+L_{1} L_{2}+\frac{L_{1}^{3}}{3}\right) \\
& Q_{4}=-\operatorname{Tr}\left(L_{4}+\frac{1}{2} L_{2}^{2}+L_{1}^{2} L_{2}+L_{1} L_{3}+\frac{1}{4} L_{1}^{4}\right), \ldots
\end{aligned}
$$

More generally, the $Q_{n}$ 's are weight-homogeneous polynomials of degree $n$ in the $L_{i}$, with weight $\left(L_{i}\right)=i$, having the form below, which can further be expressed in 
terms of the $K_{i}^{(r)}$ and $R$, using expression 7.7 for the $L_{i}$,

$$
\begin{aligned}
Q_{n} & =-\operatorname{Tr} L_{n}+\operatorname{Tr} P_{n}\left(L_{1}, L_{2}, \ldots, L_{n-1}\right) \\
& =-\operatorname{Tr} K_{n}^{(r)}-\operatorname{Tr}\left(R K_{n}^{(r)}\right)+\operatorname{Tr} P_{n}\left(L_{1}, L_{2}, \ldots, L_{n-1}\right) \\
& =-\operatorname{Tr} K_{n}^{(r)}+\operatorname{Tr} S_{n}\left(K_{1}^{(r)}, \ldots, K_{n-1}^{(r)}, R\right),
\end{aligned}
$$

with $P_{k}$ and $S_{k}$ polynomials of non-commutative variables with no linear or independent terms, but with quadratic terms and higher. From Lemma 2.2, the kernels $K_{i}^{(r)}$ and hence the $L_{i}$ are polynomials in $r$ of degree $i$ having no constant terms; hence the $Q_{n}$ 's, by their weight-homogeneity, are polynomials of degree $n$ in $r$, having no constant term and so (here one must indicate the $r$-dependence)

$$
Q_{r}(t, x):=Q(t, x)=\sum_{n=0}^{\infty}\left(\frac{r}{t}\right)^{n} \sum_{i=0}^{n-1} \frac{Q_{n, n-i}(x)}{r^{i}} .
$$

The claim is that only the terms $Q_{n, n-2 i}(x)$ appear. Observe from 0.9$)$ and 0.8$)$, that $K_{-t}^{(-r)}(u, v)=K_{t}^{(r)}(v, u)$, and thus

$$
\begin{aligned}
Q_{r}(t, x)=\operatorname{det}\left(I-K_{t}^{(r)}(u, v)\right)_{x}=\operatorname{det}\left(I-K_{t}^{(r)}(v, u)\right)_{x} & =\operatorname{det}\left(I-K_{-t}^{(-r)}(v, u)\right)_{x} \\
& =Q_{-r}(-t, x) .
\end{aligned}
$$

So from (7.10), one has

$$
Q_{r}(t, x)=\frac{1}{2}\left(Q_{r}(t, x)+Q_{-r}(-t, x)\right),
$$

implying that only the even terms appear in the sum $\sum_{i}$ in 7.10 , thus proving the statement (7.6).

Next we now proceed to estimate the two traces above:

- At first

$$
\operatorname{Tr} S_{k}\left(K_{1}^{(r)}, \ldots, K_{k-1}^{(r)}, R\right)=O\left(A^{4}\right),
$$

which we now illustrate on a typical example, like $\operatorname{Tr}\left(R K_{1}^{(r)}\right)$. Note $A(u) \geq 0$ for $u \geq 0$, and so by Lemma 7.4 .

$$
\begin{aligned}
\left|\operatorname{Tr} R K_{1}^{(r)}\right| & =\left|\operatorname{Tr}\left(\left(R-K^{(0)}\right) K_{1}^{(r)}\right)\right|+\left|\operatorname{Tr}\left(K^{(0)} K_{1}^{(r)}\right)\right| \\
& \leq\left(\left\|R-K^{(0)}\right\|_{\infty}+\left\|K^{(0)}\right\|_{\infty}\right) \iint_{x}^{\infty}\left|K_{1}^{(r)}(u, v)\right| d u d v \\
& \leq\left(O\left(A^{4}\right)+O\left(A^{2}\right)\right) \iint_{x}^{\infty} A(u) A(v) d u d v \\
& \leq O\left(A^{2}\right)\left(\int_{x}^{\infty} A(u) d u\right)^{2} \\
& \leq O\left(A^{4}\right)
\end{aligned}
$$

the last estimate follows from Lemma 7.3. More generally, the trace of a monomial of degree $\ell$ has order $O\left(A^{2 \ell}\right)$. 
- Then we evaluate $\operatorname{Tr} K_{n}^{(r)}$; in order to do so, it suffices to evaluate the kernels $K_{n}^{(r)}$ of Lemma 2.2 along the diagonal, and to notice that a skew-symmetric operator vanishes on the diagonal. Since the domain of the operator is unbounded, one needs to consider $K_{n}^{(r)} \chi_{(x, m)}$; the trace is then obtained by integrating on the diagonal and by taking the limit $m \rightarrow \infty$, upon using the decay of the kernel at $\infty$. Therefore, on the diagonal, $K_{n}^{(r)}(u, v)$ is a polynomial of degree $n$ in $r$, skipping every other term,

$$
\begin{aligned}
K_{n}^{(r)}(u, u)= & -\frac{r^{n}}{n !}\left(A^{2}(u)\right)^{(n-1)} \\
& -\frac{r^{n-2}}{(n-3) !}\left(\frac{3 n-1}{24}\left(A^{2}(u)\right)^{(n-1)}-\frac{n-1}{2}\left(A^{\prime 2}(u)\right)^{(n-3)}\right)+\ldots
\end{aligned}
$$

That the $Q_{i} \rightarrow 0$ and that $Q_{0}=\log \mathbb{P}(\sup \mathscr{A}(t) \leq x)$ has all their derivatives $\rightarrow 0$ for $x \rightarrow \infty$ follows from the statement on $T_{n, n-2 i}(A)$ (which are the coefficients of $r^{n-2 i}$ appearing in $Q_{n}(x)$ as in the statement of Lemma 7.5 ) and the asymptotics of the Airy function, from which Lemma 7.5 follows.

Proof of Theorem 7.1. From Section 6, we know that $Q(t, x)=\log \mathbb{P}\left(\sup \mathscr{A}^{(r)}(t) \leq\right.$ $x)$ satisfies the non-linear PDE 0.12 ; it is more convenient here to use version 6.7 of the equation. Also remember $Q(t, x) \rightarrow 0$, when $x \rightarrow \infty$. Then, assigning weight $=1$ to both variables $t$ and $r$, one readily checks that the PDE (6.7) can be graded as follows:

$$
\begin{aligned}
0= & \left\{\frac{\partial^{3} Q}{\partial t \partial x^{2}}, r^{2} \frac{\partial^{3} Q}{\partial x^{3}}\right\}(\text { weight }=1) \\
& +\left\{\frac{\partial^{3} Q}{\partial t \partial x^{2}}, r\left(2 \frac{\partial^{2}}{\partial t^{2}}\left(t \frac{\partial Q}{\partial x}\right)-\frac{\partial^{3}(x Q)}{\partial t \partial x^{2}}+2\left\{\frac{\partial^{2} Q}{\partial t \partial x}, \frac{\partial^{2} Q}{\partial x^{2}}\right\}\right)\right\}(\text { weight }=-1) \\
& + \text { other terms of weight }<-1 .
\end{aligned}
$$

Since, by Lemma 7.5, the solution has the following general form

$$
Q(t, x)=\sum_{0}^{\infty} \frac{Q_{n}(x)}{t^{n}}=\sum_{0}^{\infty}\left(\frac{r}{t}\right)^{n}\left(Q_{n n}(x)+\frac{1}{r^{2}} Q_{n, n-2}(x)+\frac{1}{r^{4}} Q_{n, n-4}(x)+\ldots\right)
$$

it follows that one can compute inductively all the $Q_{n n}(x)$ and then inductively all the $Q_{n, n-2}(x)$ and so the $Q_{n}$ will be as announced in Theorem 7.1 .

Setting this solution in the PDE above, yields a series of descending weights, namely $0=W_{1}+W_{-1}+W_{-3}+\ldots$, which holds for $t \rightarrow-\infty$, all $x \in \mathbb{R}$ and all integers $r>0$; this implies $W_{1}=W_{-1}=W_{-3}=\ldots=0$; one then checks the explicit 
expressions

$$
\begin{aligned}
& W_{1}=-\sum_{n=1}^{\infty} \frac{r^{n+2}}{t^{n+1}} \sum_{j=0}^{n-1}\left\{(n-j) Q_{n-j, n-j}^{\prime \prime}, Q_{j j}^{\prime \prime \prime}\right\} \\
& W_{-1}=-\sum_{n=1}^{\infty} \frac{r^{n}}{t^{n+1}} \sum_{j=0}^{n-1}\left(\begin{array}{l}
\left\{(n-j) Q_{n-j, n-j}^{\prime \prime},\left(\begin{array}{l}
Q_{j, j-2}^{\prime \prime \prime}+2(j-1)^{2} Q_{j-1, j-1}^{\prime} \\
+(j-1) x Q_{j-1, j-1}^{\prime \prime} \\
-2 \sum_{\ell+k=j-1}^{\prime \prime} \ell\left\{Q_{\ell, \ell}^{\prime}, Q_{k k}^{\prime \prime}\right\}
\end{array}\right)\right\} . \\
+\left\{(n-j) Q_{n-j, n-j-2}^{\prime \prime}, Q_{j j}^{\prime \prime \prime}\right\}
\end{array}\right\}
\end{aligned}
$$

Since this holds for all $t \searrow-\infty$ and $r>0$ integer, one must have for all $x$

$$
\sum_{j=0}^{n-1}(n-j)\left\{Q_{n-j, n-j}^{\prime \prime}, Q_{j j}^{\prime \prime \prime}\right\}=0, \quad n=1,2, \ldots
$$

with $Q_{00}=Q_{0}(x)$.

- For $n=1$, this is $\left\{Q_{11}^{\prime \prime}, Q_{0}^{\prime \prime \prime}\right\}=0$, leading to $Q_{11}=c_{0} Q_{0}^{\prime}+\alpha x+\beta$. Considering the asymptotics for $x \nearrow_{\infty}$ and using Lemmas 7.2 and 7.5, $\alpha$ and $\beta$ must $=0$, leading to the equation

$$
0=Q_{11}-c_{0} Q_{0}^{\prime}=\left(A^{\prime 2}-A A^{\prime \prime}\right)\left(1-c_{0}\right)+O\left(A^{3}\right)=\left(1-c_{0}\right) O\left(A^{2}\right)+O\left(A^{3}\right),
$$

implying $c_{0}=1$ and so $Q_{11}=Q_{0}^{\prime}$.

- For $n=2$, the equation, with the previous data introduced, reads

$$
0=\left\{2 Q_{22}^{\prime \prime}, Q_{0}^{\prime \prime \prime}\right\}+\left\{Q_{11}^{\prime \prime}, Q_{11}^{\prime \prime \prime}\right\}=\left\{Q_{0}^{\prime \prime \prime},-2 Q_{22}^{\prime \prime}+Q_{0}^{i v}\right\},
$$

which upon solving leads to $Q_{22}=\frac{1}{2} Q_{0}^{\prime \prime}+c_{1} Q_{0}^{\prime}+\alpha^{\prime} x+\beta^{\prime}$. For the same reason as before $\alpha^{\prime}=\beta^{\prime}=0$. Then again using Lemma 7.2 and Lemma 7.5, one finds for $x \nearrow \infty$,

$$
\begin{aligned}
0=Q_{22}-\frac{1}{2} Q_{0}^{\prime \prime}-c_{1} Q_{0}^{\prime} & =-\frac{A^{2}}{2}+\frac{A^{2}}{2}-c_{1}\left(A^{\prime 2}-A^{\prime \prime} A\right)+O\left(A^{3}\right) \\
& =c_{1} O\left(A^{2}\right)+O\left(A^{3}\right)
\end{aligned}
$$

implying $c_{1}=0$ and thus $Q_{22}=\frac{1}{2} Q_{0}^{\prime \prime}$.

- By induction, assume $Q_{i i}=\frac{1}{i !} Q_{0}^{(i)}$, for $0 \leq i \leq n-1$. Then substituting this identity into equation 7.11 and setting $Q_{n n}=\frac{1}{n !} Q_{0}^{(n)}+R_{n}$ leads to pairwise cancellations in equation (7.11) with only one remaining contribution $\left\{Q_{0}^{\prime \prime \prime}, R_{n}^{\prime \prime}\right\}=0$, with solution $R_{n}=c_{n} Q_{0}^{\prime}+\alpha^{\prime \prime} x+\beta^{\prime \prime}$, where $\alpha^{\prime \prime}=\beta^{\prime \prime}=0$, and thus, by the asymptotics of Lemmas 7.2 and 7.5 ,

$$
\begin{aligned}
0=Q_{n n}-\frac{1}{n !} Q_{0}^{(n)}-c_{n} Q_{0}^{\prime} & =-\frac{1}{n !}\left(A^{2}\right)^{(n-2)}+\frac{1}{n !}\left(A^{2}\right)^{(n-2)}-c_{n}\left(A^{\prime 2}-A A^{\prime \prime}\right)+O\left(A^{3}\right) \\
& =c_{n} O\left(A^{2}\right)+O\left(A^{3}\right)
\end{aligned}
$$


leading to $c_{n}=0$, completing the proof that $Q_{n n}=\frac{1}{n !} Q_{0}^{(n)}$ for all $n=1,2, \ldots$. This proves the form of the leading term (coefficient of $r^{n}$ ) in formulae (7.1) for the $Q_{n}$ 's. Since from Lemma 2.2, from the form (7.9) of the $Q_{n}$ and the fact that the coefficients $K_{n}^{(r)}(u, v)$ in the expansion of $K^{(r)}$ are divisible by $r$, the $Q_{i}$ themselves are divisible by $r$. Since they skip every other degree in $r$, this shows the formulae for $Q_{1}$ and $Q_{2}$; in particular $Q_{20}=0$.

Setting this information $Q_{n n}=\frac{1}{n !} Q_{0}^{(n)}$ into the equation $W_{-1}=0$ and noticing that the following term vanishes automatically, $\sum_{\ell+k=j-1} \ell\left\{Q_{\ell \ell}^{\prime}, Q_{k k}^{\prime \prime}\right\}=0$, one finds for $n \geq 3$,

$$
\begin{aligned}
0= & \sum_{j=2}^{n-1}\left\{\frac{Q_{0}^{(n-j+2)}}{(n-j-1) !},\left(Q_{j, j-2}^{\prime}+\frac{1}{(j-2) !}\left(x Q_{0}^{(j-1)}+2(j-2) Q_{0}^{(j-2)}\right)\right)^{\prime \prime}\right\} \\
& -\sum_{j=0}^{n-1}\left\{\frac{Q_{0}^{(j+3)}}{j !},(n-j) Q_{n-j, n-j-2}^{\prime \prime}\right\} \\
= & \sum_{j=0}^{n-3}\left\{\frac{Q_{0}^{(j+3)}}{j !},\left(\begin{array}{c}
Q_{n-j-1, n-j-3}^{\prime}-(n-j) Q_{n-j, n-j-2} \\
+\frac{1}{(n-j-3) !}\left(x Q_{0}^{(n-j-2)}+2(n-j-3) Q_{0}^{(n-j-3)}\right)
\end{array}\right)\right\}
\end{aligned}
$$

- For $n=3$, by using the fact that $Q_{20}=0$, the equation reads $\left\{Q_{0}^{\prime \prime \prime},\left(x Q_{0}^{\prime}-3 Q_{31}\right)^{\prime \prime}\right\}$ $=0$ yielding $Q_{31}=\left(\frac{x}{3}+c_{3}^{\prime}\right) Q_{0}^{\prime}+\alpha^{\prime \prime \prime} x+\beta^{\prime \prime \prime}$ with $\alpha^{\prime \prime \prime}=\beta^{\prime \prime \prime}=0$. Thus, using Lemmas 7.5 and 7.2 , and the identity 7.3 ,

$$
\begin{aligned}
0 & =Q_{31}-\left(\frac{x}{3}+c_{3}^{\prime}\right) Q_{0}^{\prime} \\
& =-\frac{1}{3}\left(\left(A^{2}\right)^{\prime}+3 \int_{x}^{\infty} A^{\prime 2}(u) d u\right)-\left(\frac{x}{3}+c_{3}^{\prime}\right)\left(A^{\prime 2}-A A^{\prime \prime}\right)+O\left(A^{3}\right) \\
& =-\left(\int_{x}^{\infty} A^{\prime 2}(u) d u+\frac{1}{3}\left(\left(A^{2}\right)^{\prime}+x\left(A^{\prime 2}-A A^{\prime \prime}\right)\right)\right)-c_{3}^{\prime}\left(A^{\prime 2}-A A^{\prime \prime}\right)+O\left(A^{3}\right) \\
& =-c_{3}^{\prime}\left(A^{\prime 2}-A A^{\prime \prime}\right)+O\left(A^{3}\right)=c_{3}^{\prime} \mathbf{O}\left(A^{2}\right)+O\left(A^{3}\right), \text { using }
\end{aligned}
$$

yielding $c_{3}^{\prime}=0$, and thus $Q_{31}=\frac{x}{3} Q_{0}^{\prime}$.

- For $n=4$, using the formula for $Q_{31}$, the equation reads

$$
0=\left\{Q_{0}^{\prime \prime \prime},\left(-4 Q_{42}+\frac{1}{3}\left(x Q_{0}^{\prime}\right)^{\prime}+x Q_{0}^{\prime \prime}+2 Q_{0}^{\prime}\right)^{\prime \prime}\right\}
$$


with solution $Q_{42}=\frac{1}{3} x Q_{0}^{\prime \prime}+\left(\frac{7}{12}+c_{4}^{\prime}\right) Q_{0}^{\prime}+\alpha^{i v} x+\beta^{i v}$ and thus $\alpha^{i v}=\beta^{i v}=0$, and by the same Lemmas 7.5 and 7.2 ,

$$
\begin{aligned}
0 & =Q_{42}-\frac{1}{3} x Q_{0}^{\prime \prime}-\left(\frac{7}{12}+c_{4}^{\prime}\right) Q_{0}^{\prime} \\
& =\frac{3}{2} A^{\prime 2}-\frac{11}{24}\left(A^{2}\right)^{\prime \prime}+\frac{1}{3} A A^{\prime \prime}-\left(\frac{7}{12}+c_{4}^{\prime}\right)\left(A^{\prime 2}-A A^{\prime \prime}\right)+O\left(A^{3}\right) \\
& =\frac{3}{2} A^{\prime 2}-\frac{11}{12}\left(A^{\prime 2}+A A^{\prime \prime}\right)+\frac{1}{3} A A^{\prime \prime}-\left(\frac{7}{12}+c_{4}^{\prime}\right)\left(A^{\prime 2}-A A^{\prime \prime}\right)+O\left(A^{3}\right) \\
& =-c_{4}^{\prime}\left(A^{\prime 2}-A A^{\prime \prime}\right)+O\left(A^{3}\right)=c_{4}^{\prime} O\left(A^{2}\right)+O\left(A^{3}\right),
\end{aligned}
$$

implying $c_{4}^{\prime}=0$.

- Using induction, assume

$$
Q_{i, i-2}=\frac{x}{3(i-3) !} Q_{0}^{(i-2)}+\frac{7}{12(i-4) !} Q_{0}^{(i-3)}
$$

holds for $i=3, \ldots, n-1$. Then setting

$$
Q_{n, n-2}=\frac{x}{3(n-3) !} Q_{0}^{(n-2)}+\frac{7}{12(n-4) !} Q_{0}^{(n-3)}+R_{n}
$$

into equation (7.12) gives the simple equation for $R_{n}$, namely $\left\{Q_{0}^{\prime \prime \prime}, R_{n}^{\prime \prime}\right\}=0$, and so $R_{n}=c_{n}^{\prime} Q_{0}^{\prime}$. Then, rewriting 7.13 and using the asymptotics for $Q_{n, n-2}$ (Lemma 7.5), and for the derivatives $Q_{0}^{(i)}$ (Lemma 7.2), and using the ODE for the Airy function $x A=A^{\prime \prime}$, we get

$$
\begin{aligned}
0= & Q_{n, n-2}-\frac{1}{12(n-3) !}\left(4 x Q_{0}^{\prime \prime}+(3 n-5) Q_{0}^{\prime}\right)^{(n-4)}-c_{n}^{\prime} Q_{0}^{\prime} \\
= & \frac{1}{12(n-3) !}\left(\begin{array}{c}
6(n-1) A^{\prime 2}-(3 n-1)\left(A^{\prime 2}+A A^{\prime \prime}\right) \\
+4 A A^{\prime \prime}-(3 n-5)\left(A^{\prime 2}-A A^{\prime \prime}\right)
\end{array}\right){ }^{(n-4)} \\
& -c_{n}^{\prime}\left(A^{\prime 2}-A A^{\prime \prime}\right)+O\left(A^{3}\right) \\
= & -c_{n}^{\prime}\left(A^{\prime 2}-A A^{\prime \prime}\right)+O\left(A^{3}\right)=c_{n}^{\prime} O\left(A^{2}\right)+O\left(A^{3}\right),
\end{aligned}
$$

implying $c_{n}^{\prime}=0$. Thus the $Q_{n}$ 's are as announced in Theorem 7.1, namely

$$
Q_{n}=\frac{r^{n}}{n !} Q_{0}^{(n)}+\frac{r^{n-2} x}{3} \frac{Q_{0}^{(n-2)}}{(n-3) !}+\frac{7 r^{n-2}}{12} \frac{Q_{0}^{(n-3)}}{(n-4) !}+r^{n-4} G(x)+\left(\begin{array}{l}
\text { lower degree } \\
\text { terms in } r
\end{array}\right) .
$$

In the same fashion we compute $Q_{51}$ and $Q_{62}$; for example, setting

$$
Q_{5}=\frac{r^{5}}{5 !} Q_{0}^{(v)}+\frac{r^{3} x}{3} \frac{Q_{0}^{\prime \prime \prime}}{2 !}+\frac{7 r^{3}}{12} \frac{Q_{0}^{\prime \prime}}{1 !}+r Q_{51}
$$

into the equation (6.7), one finds the following differential equation for $Q_{51}$, namely

$$
\left\{Q_{0}^{\prime \prime \prime}, 5 Q_{51}^{\prime \prime}-8 x Q_{0}^{\prime \prime}+4 Q_{0}^{\prime \prime 2}\right\}+2 Q_{0}^{\prime \prime \prime 2}\left(Q_{0}^{\prime \prime}+x\right)=0,
$$


which upon solving leads to

$$
Q_{51}=\frac{1}{5}\left(\begin{array}{l}
\left(x^{2}+c_{5}\right) Q_{0}^{\prime}+4 x Q_{0}+Q_{0}^{\prime 2} \\
+10 \int_{x}^{\infty} Q_{0}-6 \int_{x}^{\infty} d y \int_{y}^{\infty} d u Q_{0}^{\prime \prime 2}
\end{array}\right)=: \frac{1}{5}\left(\mathscr{F}_{5}+c_{5} Q_{0}^{\prime}\right),
$$

with a constant $c_{5}$, which has been shown by Aminul Huq (private communication, 2008) to be 0 . Similarly one finds a differential equation for $Q_{62}$ and upon solving one finds, for some integration constant $c_{6}$,

$$
Q_{62}=\frac{1}{5}\left(\mathscr{F}_{5}^{\prime}+\frac{5}{18}\left(x^{2} Q_{0}^{\prime \prime}+13\left(x+c_{6}\right) Q_{0}^{\prime}\right)\right) .
$$

Assembling all the pieces, one notices that two Taylor series in $Q_{0}$ and $Q_{0}^{\prime}$ make their appearance in the $1 / t$-expansion of $Q(t, x)$, leading to shifts in the argument of $Q_{0}(x)$ up to order 5:

$$
\begin{aligned}
Q(t, x)= & \sum_{0}^{\infty} \frac{Q_{i}(x)}{t^{i}} \\
= & \sum_{0}^{\infty}\left(\frac{r}{t}\right)^{n} \frac{Q_{0}^{(n)}(x)}{n !}+\left(\frac{x r}{3 t^{3}}+\frac{7 r^{2}}{12 t^{4}}\right) \sum_{0}^{\infty}\left(\frac{r}{t}\right)^{n} \frac{Q_{0}^{(1+n)}(x)}{n !} \\
& +\frac{r}{5 t^{5}} \mathscr{F}_{5}+O\left(\frac{1}{t^{6}}\right) \\
= & Q_{0}\left(x+\frac{r}{t}\right)+\left(\frac{x r}{3 t^{3}}+\frac{7 r^{2}}{12 t^{4}}\right) Q_{0}^{\prime}\left(x+\frac{r}{t}\right)+\frac{r}{5 t^{5}} \mathscr{F}_{5}+O\left(\frac{1}{t^{6}}\right) \\
= & \left.Q_{0}\left(x+\frac{r}{t}+\frac{x r}{3 t^{3}}+\frac{7 r^{2}}{12 t^{4}}\right)+\frac{r}{5 t^{5}} \mathscr{F}_{5}+c_{5} Q_{0}^{\prime}\right)+O\left(\frac{1}{t^{6}}\right) .
\end{aligned}
$$

Exponentiating 7.14), remembering that $e^{Q_{0}(y)}=\mathbb{P}(\sup \mathscr{A}(t) \leq y)$, taking a derivative $\frac{d}{d y} \mathbb{P}(\sup \mathscr{A}(t) \leq y)$ and setting $P_{0}(x):=\mathbb{P}(\sup \mathscr{A}(t) \leq x)$ yields

$$
\begin{aligned}
\frac{d}{d x} \mathbb{P}\left(\sup \mathscr{A}^{(r)}(t) \leq x\right)= & \left.\left(1+\frac{r}{3 t^{3}}\right) \frac{d}{d y} \mathbb{P}(\sup \mathscr{A}(t) \leq y)\right|_{y=\left(x+\frac{r}{t}\right)\left(1+\frac{r}{3 t^{3}}\right)+\frac{r^{2}}{4 t^{4}}}+O\left(\frac{1}{t^{5}}\right) \\
= & P_{0}^{\prime}+\frac{r}{t} P_{0}^{\prime \prime}+\frac{r^{2}}{2 t^{2}} P_{0}^{\prime \prime \prime}+\frac{r}{6 t^{3}}\left(r^{2} P_{0}^{(i v)}+\left(2 x P_{0}^{\prime}\right)^{\prime}\right) \\
& +\frac{r^{2}}{24 t^{4}}\left(r^{2} P_{0}^{(v)}+14 P_{0}^{\prime \prime}+8\left(x P_{0}^{\prime \prime}\right)^{\prime}\right)+O\left(\frac{1}{t^{5}}\right) .
\end{aligned}
$$

For the moments with regard to the density $\frac{d}{d x} \mathbb{P}\left(\sup \mathscr{A}^{(r)}(t) \leq x\right)$,

$$
\mu_{\ell}^{(r)}(t)=\int_{-\infty}^{\infty} x^{\ell} \frac{d}{d x} \mathbb{P}\left(\sup \mathscr{A}^{(r)}(t) \leq x\right) d x
$$

one reads off from 7.15 the following expansion for $\mu_{\ell}^{(r)}(t)$ in terms of the $t$ independent moments

$$
\mu_{\ell}=\int_{-\infty}^{\infty} x^{\ell} \frac{d}{d x} \mathbb{P}(\sup \mathscr{A}(t) \leq x) d x=\int_{-\infty}^{\infty} x^{\ell} P_{0}^{\prime}(x) d x
$$


namely,

$$
\begin{aligned}
\mu_{\ell}^{(r)}(t)= & \mu_{\ell}-\ell \frac{r}{t} \mu_{\ell-1}+\frac{r^{2}}{2 t^{2}} \ell(\ell-1) \mu_{\ell-2}+\frac{r}{6 t^{3}}\left(-r^{2} \ell(\ell-1)(\ell-2) \mu_{\ell-3}-2 \ell \mu_{\ell}\right) \\
& +\frac{r^{2}}{24 t^{4}}\left(r^{2} \ell(\ell-1)(\ell-2)(\ell-3) \mu_{\ell-4}+\ell(8 \ell-14) \mu_{\ell-1}\right)+O\left(\frac{1}{t^{5}}\right) .
\end{aligned}
$$

In particular, the mean and second moment behave as

$$
\mu_{1}^{(r)}(t)=\mu_{1}-\frac{r}{t}-\frac{r \mu_{1}}{3 t^{3}}-\frac{r^{2}}{4 t^{4}}+O\left(\frac{1}{t^{5}}\right)
$$

and

$$
\mu_{2}^{(r)}(t)=\mu_{2}-\frac{2 r \mu_{1}}{t}+\frac{r^{2}}{t^{2}}-\frac{2 r \mu_{2}}{3 t^{3}}+\frac{r^{2} \mu_{1}}{6 t^{4}}+O\left(\frac{1}{t^{5}}\right) .
$$

Hence the variance of the right edge of the process behaves as

$$
\begin{aligned}
\operatorname{var}\left(\sup \mathscr{A}^{(r)}(t)\right)=\left(\mu_{2}^{(r)}-\mu_{1}^{(r) 2}\right)(t) & =\left(\mu_{2}-\mu_{1}^{2}\right)\left(1-\frac{2 r}{3 t^{3}}\right)+O\left(\frac{1}{t^{5}}\right) \\
& =\operatorname{var}\left(\sup \mathscr{A}^{(0)}(t)\right)\left(1-\frac{2 r}{3 t^{3}}\right)+O\left(\frac{1}{t^{5}}\right),
\end{aligned}
$$

ending the proof of Theorem 7.1 .

\section{The $r$-Airy process, an interpolation between the Airy and Pearcey processes}

Consider $n$ non-intersecting Brownian motions on $\mathbb{R}$, with $0<p<1$ and $b<a$ :

$$
\mathbb{P}_{n}^{(a, b)}\left(\begin{array}{l|c}
\text { all } x_{j}(t) \in E \text { for } 1 \leq j \leq n & \begin{array}{c}
\text { all } x_{j}(0)=0 \\
p n \text { paths end up at } a \text { at } t=1 \\
(1-p) n \text { paths end up at } b \text { at } t=1
\end{array}
\end{array}\right)
$$

It is intuitive that, when $n \rightarrow \infty$, the mean density of Brownian particles has its support on one interval for $t \sim 0$ and on two intervals for $t \sim 1$, so that a bifurcation appears for some intermediate time $t_{0}$, where one interval splits into two intervals. At this point the boundary of the support of the mean density in $(x, t)$-space has a cusp; see Figure 0.3. The Pearcey process describes this cloud of particles near the point of bifurcation, with time and space stretched in such a way that the outer particles appear infinitely far and such that the time horizon $t=1$ is at infinity. In [1] it is shown that the same Pearcey process appears in the neighborhood of this cusp, independently of the target points $a$ and $b$, and the number $n p$ of paths forced to $a$, showing "universality" of the Pearcey process. It is convenient to introduce the parametrization of $p$,

$$
p=\frac{1}{1+q^{3}} \text { with } 0<q<\infty .
$$

Setting for simplicity $b=0$, one has the following: 
Proposition 8.1. [1] For $n \rightarrow \infty$, the cloud of Brownian particles lies within a region, having a cusp at location $\left(x_{0} \sqrt{n}, t_{0}\right)$, with

$$
x_{0}=\frac{(2 q-1) a}{q+1} t_{0}, \quad t_{0}=\left(1+2 a^{2} \frac{q^{2}-q+1}{(q+1)^{2}}\right)^{-1} .
$$

Moreover, the following Brownian motion probability tends to the probability for the Pearcey process $\mathscr{P}(t)$ :

$\lim _{n \rightarrow \infty} \mathbb{P}_{B R}^{(0, a \sqrt{n})}\left(\right.$ all $\left.x_{j}\left(t_{0}+\left(c_{0} \mu\right)^{2} \frac{2 \tau}{n^{1 / 2}}\right) \in x_{0} n^{1 / 2}+c_{0} A \tau+c_{0} \mu \frac{E^{c}}{n^{1 / 4}}\right)$

$$
=\mathbb{P}^{\mathscr{P}}(\mathscr{P}(\tau) \cap E=\emptyset),
$$

with constants expressed in terms of 8.1) and (8.2),

$$
\begin{gathered}
\mu:=\left(\frac{q^{2}-q+1}{q}\right)^{1 / 4}>0, \quad c_{0}:=\sqrt{\frac{t_{0}\left(1-t_{0}\right)}{2}}>0, \\
A:=q^{1 / 2}\left(1-\frac{x_{0}}{a}\right)-q^{-1 / 2} \frac{x_{0}}{a} .
\end{gathered}
$$

The $r$-Airy process is an interpolation between the Pearcey process and the Airy process, which can easily be described by looking at Figure 0.3:

Theorem 8.2. When $p \rightarrow 0$ and for $n$ very large, such that pn equals a fixed integer $r>0$, the tip $\left(x_{0} \sqrt{n}, t_{0}\right)$ of the cusp (as given by (8.2)) moves towards the right hand boundary of the picture, and, in particular, to the tangency point of the line through $(a \sqrt{n}, 1)$ tangent to the curve $y=\sqrt{2 n t(1-t)}$ :

$$
\left(x_{0} \sqrt{n}, t_{0}\right) \rightarrow\left(\frac{2 a}{1+2 a^{2}} \sqrt{n}, \frac{1}{1+2 a^{2}}\right) \in \text { curve }\{y=\sqrt{2 n t(1-t)}\} .
$$

Also the Pearcey process near the cusp tends to the r-Airy process in the neighborhood of the point of tangency above.

Proof. Indeed, letting $p \rightarrow 0$, or what is the same from [8.1), letting $q \rightarrow \infty$, one sees from formula $(8.2)$ that the cusp is located at the point

$$
x_{0} \sqrt{n}=2 a t_{0} \sqrt{n} \text { and } t_{0}=\frac{1}{1+2 a^{2}} .
$$

This implies that the point $(y, t)=\left(x_{0} \sqrt{n}, t_{0}\right)$ belongs to the curve $y=\sqrt{2 n t(1-t)}$ and that

$$
a \sqrt{n}=\sqrt{\frac{1-t_{0}}{2 t_{0}}} \sqrt{n}=\rho_{0} \sqrt{\frac{n}{2}},
$$

establishing the first part of Theorem 8.2. That the Pearcey process tends to the $r$-Airy process will be done elsewhere. 


\section{Appendix}

The purpose of this appendix is to show that the first few $Q_{i}$ of Theorem 7.1 can be obtained, with tears, by functional analytical methods, in the style of Widom [31]. The proof requires many intricate identities involving the kernels $K_{i}^{(r)}$, some of which can be found in Tracy-Widom [26]. This section should convince the reader of the usefulness of the PDE's in computing the asymptotics for $t \rightarrow-\infty$.

Remember the $L_{i}=\left(I+R_{x}\right) K_{i}^{(r)}$ from 7.7 , where we now indicate the explicit dependency of the resolvent $R_{x}=K^{(0)}\left(I-K^{(0)}\right)^{-1}$ on $x$, since all operators act on $L^{2}(x, \infty)$. Then $Q(t, x)$ has an expansion, with $Q_{i}$ 's given in 7.8 ,

$$
Q=\operatorname{Tr} \log \left(I-K_{t}^{(r)}\right)=\sum_{i=0}^{\infty} \frac{Q_{i}}{t^{i}}=\operatorname{Tr} \log \left(I-K^{(0)}\right)+\sum_{i=1}^{\infty} \frac{Q_{i}}{t^{i}} .
$$

Throughout this section, we shall be using the inner-product

$$
\langle f, g\rangle:=\int_{\mathbb{R}} \chi_{(x, \infty)}(u) f(u) g(u) d u .
$$

\section{Proposition 9.1.}

$$
Q_{1}=r Q_{0}^{\prime}, Q_{2}=\frac{r^{2}}{2 !} Q_{0}^{\prime \prime}, Q_{3}=\frac{r^{3}}{3 !} Q_{0}^{\prime \prime \prime}+\frac{r}{3} x Q_{0}^{\prime} .
$$

\section{Lemma 9.2.}

$$
\operatorname{Tr} L_{1}^{n}=(-r)^{n}\left\langle\left(I+R_{x}\right) A, A\right\rangle^{n} .
$$

Proof. Indeed,

$$
\begin{aligned}
& \operatorname{Tr}\left(\left(I+R_{x}\right) K_{1}\right)^{n} \\
& =(-r)^{n} \int_{(x, \infty)^{n}} d u_{1} \ldots d u_{n}\left(\left(\left(I+R_{x}\right) A\left(u_{1}\right)\right) A\left(u_{2}\right)\right)\left(\left(\left(I+R_{x}\right) A\left(u_{2}\right)\right) A\left(u_{3}\right)\right) \\
& \quad \ldots\left(\left(\left(I+R_{x}\right) A\left(u_{n}\right)\right) A\left(u_{1}\right)\right) \\
& =(-r)^{n}\left\langle\left(I+R_{x}\right) A(u), A(u)\right\rangle^{n} .
\end{aligned}
$$

The identities in the following Lemma can be found in or deduced from TracyWidom [26].

\section{Lemma 9.3.}

(9.2) $\left(\frac{\partial}{\partial x}+\frac{\partial}{\partial u}+\frac{\partial}{\partial v}\right) R_{x}(u, v)=-\left(\left(I+R_{x}\right) A(u)\right)\left(\left(I+R_{x}\right) A(v)\right)$

(9.3) $\left(\frac{\partial}{\partial x}+\frac{\partial}{\partial u}\right)\left(I+R_{x}\right) A(u)=\left(I+R_{x}\right) A^{\prime}(u)-\left(I+R_{x}\right) A(u)\left\langle\left(I+R_{x}\right) A, A\right\rangle$. 


$$
\begin{gathered}
2\left\langle\left(I+R_{x}\right) A^{\prime}, A\right\rangle-\left\langle\left(I+R_{x}\right) A, A\right\rangle^{2}=-\left(\left(I+R_{x}\right) A(x)\right)^{2} \\
2\left\langle\left(I+R_{x}\right) A, A^{\prime \prime}\right\rangle-\left\langle\left(I+R_{x}\right) A^{\prime}, A^{\prime}\right\rangle=x\left\langle\left(I+R_{x}\right) A, A\right\rangle \\
\frac{d}{d x}\left(I+R_{x}\right) A^{\prime}(u)=-R_{x}(u, x)\left(I+R_{x}\right) A^{\prime}(x) \\
\frac{\partial}{\partial u}\left(I+R_{x}\right) A^{\prime}(u)=u\left(I+R_{x}\right) A(u)-2\left\langle\left(I+R_{x}\right) A^{\prime}, A\right\rangle\left(I+R_{x}\right) A(u) \\
+\left\langle\left(I+R_{x}\right) A, A\right\rangle\left(I+R_{x}\right) A^{\prime}(u)+R(u, x)\left(I+R_{x}\right) A^{\prime}(x) .
\end{gathered}
$$

Lemma 9.4. We have

$$
\begin{aligned}
Q_{0}^{\prime}(x) & =\left\langle\left(I+R_{x}\right) A, A\right\rangle \quad \text { and } \quad Q_{0}^{\prime \prime}(x)=-\left(\left(I+R_{x}\right) A(x)\right)^{2} \\
\frac{1}{2} Q_{0}^{\prime \prime \prime}(x) & =-\left(\left(I+R_{x}\right) A(x)\right)\left(\left(I+R_{x}\right) A^{\prime}(x)\right)+\left(\left(I+R_{x}\right) A(x)\right)^{2}\left\langle\left(I+R_{x}\right) A, A\right\rangle .
\end{aligned}
$$

Proof. One computes

$$
\begin{aligned}
& Q_{0}^{\prime}(x)= \frac{\partial}{\partial x} \operatorname{Tr} \log \left(I-K^{(0)} \chi(x, \infty)\right) \\
&=-\frac{\partial}{\partial x} \operatorname{Tr}\left(K^{(0)}(u, v)+\frac{1}{2} \int_{x}^{\infty} K^{(0)}(u, w) K^{(0)}(w, v) d w+\ldots\right) \\
&=-\frac{\partial}{\partial x}\left(\int_{x}^{\infty} K^{(0)}(u, u) d u+\frac{1}{2} \int_{x}^{\infty} \int_{x}^{\infty} K^{(0)}(u, w) K^{(0)}(w, u) d u d w\right. \\
&\left.+\frac{1}{3} \int_{x}^{\infty} \int_{x}^{\infty} \int_{x}^{\infty} K_{0}\left(u, w_{1}\right) K_{0}\left(w_{1}, w_{2}\right) K^{(0)}\left(w_{2}, u\right) d u d w_{1} d w_{2}+\ldots\right) \\
&=K^{(0)}(x, x)+\int_{x}^{\infty} K^{(0)}(x, v) K^{(0)}(v, x) d v \\
&=\int_{x}^{\infty}(x, x)=\left\langle\left( I+R_{x}^{\infty} K^{(0)}\left(x, w_{1}\right) K^{(0)}\left(w_{1}, w_{2}\right) K^{(0)}\left(w_{2}, x\right) d w_{1} d w_{2}+\ldots\right.\right. \\
&
\end{aligned}
$$

by the Neumann series for $R_{x}=\left(I-K^{(0)}\right)^{-1}-I$. Moreover, using the previous result,

$$
\begin{aligned}
Q_{0}^{\prime \prime}(x)=\frac{d}{d x} R_{x}(x, x) & =\left.\left(\frac{\partial}{\partial x}+\frac{\partial}{\partial u}+\frac{\partial}{\partial v}\right) R_{x}(u, v)\right|_{u=v=x} \\
& \left.=-\left(\left(I+R_{x}\right) A(u)\right)\left(I+R_{x}\right) A(v)\right)\left.\right|_{u=v=x} \text { using (9.2) } \\
& =-\left(\left(I+R_{x}\right) A(x)\right)^{2}
\end{aligned}
$$


and again using the result just obtained and using identity 9.3 ,

$$
\begin{aligned}
Q_{0}^{\prime \prime \prime}(x) & =-2\left(\left(I+R_{x}\right) A(x)\right) \frac{d}{d x}\left(I+R_{x}\right) A(x) \\
& =-\left.2\left(\left(I+R_{x}\right) A(x)\right)\left(\frac{\partial}{\partial x}+\frac{\partial}{\partial u}\right)\left(I+R_{x}\right) A(u)\right|_{u=x} \\
& =-2\left(\left(I+R_{x}\right) A(x)\right)\left(I+R_{x}\right) A^{\prime}(x)+2\left(\left(I+R_{x}\right) A(x)\right)^{2}\left\langle\left(I+R_{x}\right) A, A\right\rangle,
\end{aligned}
$$

proving Lemma 9.4 .

\section{Lemma 9.5.}

$$
\begin{aligned}
Q_{1}(x)= & r\left\langle\left(I+R_{x}\right) A, A\right\rangle \\
Q_{2}(x)= & -\frac{r^{2}}{2}\left(\left(I+R_{x}\right) A(x)\right)^{2} \\
Q_{3}(x)= & \frac{r^{3}}{3}\left(\left(\left(I+R_{x}\right) A(x)\right)^{2}\left\langle\left(I+R_{x}\right) A, A\right\rangle-\left(I+R_{x}\right) A(x)\left(I+R_{x}\right) A^{\prime}(x)\right) \\
& +\frac{r x}{3}\left\langle\left(I+R_{x}\right) A, A\right\rangle .
\end{aligned}
$$

Proof. Indeed, by (7.8) and 2.13,

$$
\begin{aligned}
Q_{1}(x)=-\operatorname{Tr} L_{1} & =-\operatorname{Tr} K_{1}^{(r)}-\operatorname{Tr} R_{x} K_{1}^{(r)} \\
& =r \int_{x}^{\infty} d u A(u)\left(A(u)+\int_{x}^{\infty} R_{x}(u, v) A(v) d v\right) \\
& =r \int_{x}^{\infty} A(u)((I+R) A)(u) d u=r\left\langle\left(I+R_{x}\right) A, A\right\rangle .
\end{aligned}
$$

Computing $Q_{2}$ by (7.8) involves $\operatorname{Tr} L_{2}$ and $\operatorname{Tr} L_{1}^{2}$. Since $K_{2}^{(r)}(u, v)$ has a symmetric and skew-symmetric part, and since $I+R_{x}$ is symmetric, and remembering the form of $K_{2}^{(r)}(u, v)$ in 2.13 , we have (by symmetry) and the fact that a symmetric times a skew-symmetric operator is traceless,

$$
\begin{aligned}
\operatorname{Tr} L_{2}=\operatorname{Tr}\left(I+R_{x}\right) K_{2}^{(r)} & =-\frac{r^{2}}{2} \operatorname{Tr}\left(I+R_{x}\right)\left(A^{\prime}(u) A(v)+A(u) A^{\prime}(v)\right) \\
& =-r^{2}\left\langle\left(I+R_{x}\right) A^{\prime}, A\right\rangle .
\end{aligned}
$$

Hence, combining this result with the computation of $\operatorname{Tr} L_{1}^{2}$ in Lemma 9.2, one finds, using (9.4),

$$
\begin{aligned}
Q_{2}=-\operatorname{Tr}\left(L_{2}+\frac{L_{1}^{2}}{2}\right) & =\frac{r^{2}}{2}\left(2\left\langle\left(I+R_{x}\right) A^{\prime}, A\right\rangle-\left\langle\left(I+R_{x}\right) A, A\right\rangle^{2}\right) \\
& =-\frac{r^{2}}{2}\left(\left(I+R_{x}\right) A(x)\right)^{2} .
\end{aligned}
$$


The computation of $Q_{3}$, by 7.8 , involves $\operatorname{Tr} L_{3}, \operatorname{Tr} L_{1} L_{2}$ and $\operatorname{Tr} L_{1}^{3}$. Using again the fact that a symmetric times a skew-symmetric operator is traceless, one reads off from the form of $K_{3}^{(r)}$ (see 2.13 ) ) the following:

$$
\begin{aligned}
\operatorname{Tr} L_{3}=\operatorname{Tr}\left(I+R_{x}\right) K_{3}^{(r)}=-\frac{r^{3}}{3}(\langle(I+ & \left.\left.\left.R_{x}\right) A, A^{\prime \prime}\right\rangle+\left\langle\left(I+R_{x}\right) A^{\prime}, A^{\prime}\right\rangle\right) \\
& -\frac{r}{3}\left(2\left\langle\left(I+R_{x}\right) A, A^{\prime \prime}\right\rangle-\left\langle\left(I+R_{x}\right) A^{\prime}, A^{\prime}\right\rangle\right)
\end{aligned}
$$

and using

$$
\begin{aligned}
\left(I+R_{x}\right) K_{2}^{(r)}=-\frac{r^{2}}{2}(((I+ & \left.\left.\left.R_{x}\right) A^{\prime}(u)\right) A(v)+\left(\left(I+R_{x}\right) A(u)\right) A^{\prime}(v)\right) \\
& -\frac{r}{2}\left(\left(\left(I+R_{x}\right) A^{\prime}(u)\right) A(v)-\left(\left(I+R_{x}\right) A(u)\right) A^{\prime}(v)\right),
\end{aligned}
$$

one computes

$$
\begin{aligned}
\operatorname{Tr} L_{1} L_{2}= & \operatorname{Tr}\left(I+R_{x}\right) K_{1}^{(r)}\left(I+R_{x}\right) K_{2}^{(r)} \\
= & \frac{r^{3}}{2} \iint_{(x, \infty)^{2}}\left(\left(I+R_{x}\right) A\right)\left(w_{1}\right) A\left(w_{2}\right)\left(\left(I+R_{x}\right) A^{\prime}\right)\left(w_{2}\right) A\left(w_{1}\right) d w_{1} d w_{2} \\
& +\frac{r^{3}}{2} \iint\left(\left(I+R_{x}\right) A\right)\left(w_{1}\right) A\left(w_{2}\right)\left(\left(I+R_{x}\right) A\right)\left(w_{2}\right) A^{\prime}\left(w_{1}\right) d w_{1} d w_{2} \\
& +\frac{r^{2}}{2} \iint\left(\left(I+R_{x}\right) A\right)\left(w_{1}\right) A\left(w_{2}\right)\left(\left(I+R_{x}\right) A^{\prime}\right)\left(w_{2}\right) A\left(w_{1}\right) d w_{1} d w_{2} \\
& -\frac{r^{2}}{2} \iint\left(\left(I+R_{x}\right) A\right)\left(w_{1}\right) A\left(w_{2}\right)\left(\left(I+R_{x}\right) A\right)\left(w_{2}\right) A^{\prime}\left(w_{1}\right) d w_{1} d w_{2} \\
= & \frac{r^{3}}{2}\left(\begin{array}{c}
\left\langle\left(I+R_{x}\right) A, A\right\rangle\left\langle\left(I+R_{x}\right) A^{\prime}, A\right\rangle \\
+\left\langle\left(I+R_{x}\right) A, A\right\rangle\left\langle A^{\prime},\left(I+R_{x}\right) A\right\rangle
\end{array}\right) \\
& +\frac{r^{2}}{2}\left(\begin{array}{c}
\left\langle\left(I+R_{x}\right) A, A\right\rangle\left\langle\left(I+R_{x}\right) A^{\prime}, A\right\rangle \\
-\left\langle\left(I+R_{x}\right) A, A\right\rangle\left\langle A^{\prime},\left(I+R_{x}\right) A\right\rangle
\end{array}\right) \\
= & r^{3}\left\langle\left(I+R_{x}\right) A, A\right\rangle\left\langle\left(I+R_{x}\right) A^{\prime}, A\right\rangle .
\end{aligned}
$$


Putting the pieces together and using (7.8) and using Lemmas 9.2 and 9.4 , one obtains

$$
\begin{aligned}
& Q_{3}=-\operatorname{Tr}\left(L_{3}+L_{1} L_{2}+\frac{1}{3} L_{1}^{3}\right) \\
& =\frac{r^{3}}{3}\left(\begin{array}{c}
\left\langle\left(I+R_{x}\right) A, A^{\prime \prime}\right\rangle+\left\langle\left(I+R_{x}\right) A^{\prime}, A^{\prime}\right\rangle \\
-3\left\langle\left(I+R_{x}\right) A, A\right\rangle\left\langle\left(I+R_{x}\right) A^{\prime}, A\right\rangle \\
+\left\langle\left(I+R_{x}\right) A, A\right\rangle^{3}
\end{array}\right) \\
& +\frac{r}{3}\left(2\left\langle\left(I+R_{x}\right) A, A^{\prime \prime}\right\rangle-\left\langle\left(I+R_{x}\right) A^{\prime}, A^{\prime}\right\rangle\right) \\
& =\frac{r^{3}}{3}\left(\begin{array}{l}
\left\langle\left(I+R_{x}\right) A, A^{\prime \prime}\right\rangle+\left\langle\left(I+R_{x}\right) A^{\prime}, A^{\prime}\right\rangle \\
-\left\langle\left(I+R_{x}\right) A, A\right\rangle\left\langle\left(I+R_{x}\right) A^{\prime}, A\right\rangle \\
+\left\langle\left(I+R_{x}\right) A, A\right\rangle\left(\left\langle\left(I+R_{x}\right) A, A\right\rangle^{2}-2\left\langle\left(I+R_{x}\right) A^{\prime}, A\right\rangle\right)
\end{array}\right) \\
& +\frac{r}{3}\left(2\left\langle\left(I+R_{x}\right) A, A^{\prime \prime}\right\rangle-\left\langle\left(I+R_{x}\right) A^{\prime}, A^{\prime}\right\rangle\right) \\
& =\frac{r^{3}}{3}\left(-\left(I+R_{x}\right) A(x)\left(I+R_{x}\right) A^{\prime}(x)+((I+R) A(x))^{2}\left\langle\left(I+R_{x}\right) A, A\right\rangle\right) \\
& +\frac{r x}{3}\left\langle\left(I+R_{x}\right) A, A\right\rangle,
\end{aligned}
$$

using in the last equality (9.4), (9.5) combined with 9.11) below. Then, using the differential equation $u A(u)=A^{\prime \prime}(u)$, one checks:

$$
\begin{aligned}
&\left\langle\left(I+R_{x}\right) A^{\prime}, A^{\prime}\right\rangle \\
&=-A(x)\left(I+R_{x}\right) A^{\prime}(x)-\left\langle\frac{\partial}{\partial u}\left(I+R_{x}\right) A^{\prime}, A\right\rangle \text { (by integration by parts) } \\
&=-A(x)\left(I+R_{x}\right) A^{\prime}(x)-\left\langle\left(I+R_{x}\right) A(u), u A(u)\right\rangle+\left\langle\left(I+R_{x}\right) A^{\prime}, A\right\rangle\left\langle\left(I+R_{x}\right) A, A\right\rangle \\
&-\langle R(u, x), A(u)\rangle(I+R) A^{\prime}(x), \text { using }(9.7), \\
&=-\left(I+R_{x}\right) A(x)\left(I+R_{x}\right) A^{\prime}(x)-\left\langle\left(I+R_{x}\right) A, A^{\prime \prime}\right\rangle+\left\langle\left(I+R_{x}\right) A^{\prime}, A\right\rangle\left\langle\left(I+R_{x}\right) A, A\right\rangle .
\end{aligned}
$$

Proof of Proposition 9.1. The formulae follow immediately from comparing the formulae of Lemmas 9.4 and 9.5 .

Acknowledgment. Pierre van Moerbeke thanks Gérard Ben Arous for a useful conversation (May 2006) and thanks Patrik Ferrari for a very interesting discussion (January 2008) concerning Theorem 0.1. Mark Adler and Pierre van Moerbeke gratefully acknowledge the support of a National Science Foundation grant \# DMS-07-04271. This work was partially done while PvM was a member of the Miller Institute for Research in Science, Berkeley, California. The support of National Science Foundation grant \# DMS-07-0427, a European Science Foundation 
grant (MISGAM), a Marie Curie Grant (ENIGMA), a FNRS grant and a "Interuniversity Attraction Pole" (Belgium) grants are gratefully acknowledged.

\section{Bibliography}

[1] M. Adler, N. Orantin and P. van Moerbeke: Universality for the Pearcey process and a PDE for the transition probabilities, Preprint 2008.

[2] M. Adler and P. van Moerbeke: Hermitian, symmetric and symplectic random ensembles: PDE's for the distribution of the spectrum, Annals of Math., 153, 149-189 (2001).

[3] M. Adler and P. van Moerbeke: PDE's for the joint distributions of the Dyson, Airy and Sine processes, The Annals of Probability, 33, 1326-1361 (2005). (arXiv:math.PR/0302329 and math.PR/0403504)

[4] M. Adler and P. van Moerbeke: PDE's for the Gaussian ensemble with external source and the Pearcey distribution, Comm. Pure and Appl. Math, 60 1-32 (2007) (arXiv:math.PR/0509047)

[5] M. Adler and P. van Moerbeke: Joint probability for the Pearcey process, (arXiv:math/0612393).

[6] M. Adler, P. van Moerbeke and P. Vanhaecke: Moment matrices and multicomponent KP, with applications to random matrix theory, (2006) (arXiv:math-ph/0612064)

[7] A. Aptekarev, P. Bleher and A. Kuijlaars: Large $n$ limit of Gaussian random matrices with external source. II. Comm. Math. Phys. 259 367-389 (2005) (arXiv: math-ph/0408041)

[8] Jinho Baik, Gérard Ben Arous, Sandrine Péché: Phase transition of the largest eigenvalue for non-null complex sample covariance matrices, Ann. Probab. 33, no. 5, 1643-1697 (2005) (arXiv:math/0403022)

[9] Jinho Baik: Painlevé formulas of the limiting distributions for non-null complex sample covariance matrices, Duke Math. J. 133, no. 2, 205-235 (2006) (arXiv:math/0504606)

[10] P. Bleher and A. Kuijlaars: Random matrices with external source and multiple orthogonal polynomials, Internat. Math. Research Notices 3, 109-129 (2004) (arXiv:math-ph/0307055).

[11] E. Brézin and S. Hikami: Correlations of nearby levels induced by a random potential, Nuclear Physics B 479, 697-706 (1996).

[12] E. Brézin and S. Hikami: Extension of level spacing universality, Phys. Rev., E 56, 264-269 (1997).

[13] E. Brézin and S. Hikami: Universal singularity at the closure of a gap in a random matrix theory, Phys. Rev., E 57, 4140-4149 (1998).

[14] E. Brézin and S. Hikami: Level spacing of random matrices in an external source, Phys. Rev., E 58, 7176-7185 (1998).

[15] P. Deift, T. Kriecherbauer, K. T-R McLaughlin, S. Venakides and X. Zhou: Strong asymptotics of orthogonal polynomials with respect to exponential weights, Comm. of Pure and Appl. Math. 52, 1491-1552 (1999)

[16] F.J. Dyson: A Brownian-Motion Model for the Eigenvalues of a Random Matrix, Journal of Math. Phys. 3, 1191-1198 (1962)

[17] D.J. Grabiner: Brownian-Motion in a Weyl chamber, non-colliding particles and random matrices, Ann. Institut H. Poincaré 35, 177-204 (1999)

[18] S. P. Hastings and J. B. McLeod: A boundary value problem associated with the second Painlevé transcendent and Korteweg-de Vries equation, Arch. Rational Mech. Anal. 73, 31-51 (1980)

[19] K. Johansson: Universality of the Local Spacing distribution in certain ensembles of Hermitian Wigner Matrices, Comm. Math. Phys. 215, 683-705 (2001)

[20] K. Johansson: Discrete Polynuclear Growth and Determinantal Processes, Comm. Math. Phys. 242, 277-329 (2003). 
[21] K. Johansson: The Arctic circle boundary and the Airy process, Ann. Probab. 33, no. 1, 1-30 (2005) (arXiv: Math. PR/0306216)

[22] S. Karlin and J. McGregor: Coincidence probabilities, Pacific J. Math. 9, 1141-1164 (1959).

[23] A. Okounkov and N. Reshetikhin: Random skew plane partitions and the Pearcey process, Comm. Math. Phys. 269, no. 3, 571-609 (2007) (arXiv:math.CO/0503508)

[24] L.A. Pastur: The spectrum of random matrices (Russian), Teoret. Mat. Fiz. 10, 102-112 (1972).

[25] M. Prähofer and H. Spohn: Scale Invariance of the PNG Droplet and the Airy Process, J. Stat. Phys. 108, 1071-1106 (2002). (arXiv:Math. PR/0105240)

[26] C.A. Tracy and H. Widom : Level-spacing distributions and the Airy kernel, Comm. Math. Phys., 159, 151-174, (1994).

[27] C.A. Tracy and H. Widom : Differential equations for Dyson processes, Comm. Math. Phys. 252, no. 1-3, 7-41 (2004) (ArXiv:Math. PR/0309082)

[28] C. A. Tracy and H. Widom: The Pearcey Process, Comm. Math. Phys. 263, no. 2, 381-400 (2006). (arXiv:math. PR /0412005)

[29] Sandrine Péché: The largest eigenvalue of small rank perturbations of Hermitian random matrices Probab. Theory Related Fields 134, no. 1, 127-173 (2006). (arXiv:math/0411487)

[30] K. Ueno and K. Takasaki: Toda Lattice Hierarchy, Adv. Studies in Pure Math. 4, 1-95 (1984).

[31] H. Widom : On asymptotics for the Airy process, J. Statist. Phys. 115, 1129-1134 (2004). (ArXiv. Math. PR/0308157)

[32] P. Zinn-Justin: Random Hermitian matrices in an external field, Nuclear Physics B 497, 725732 (1997).

[33] P. Zinn-Justin: Universality of correlation functions in Hermitian random matrices in an external field, Comm. Math. Phys. 194, 631-650 (1998).

adler@brandeis.edu

jonathan.delepine@uclouvain.be

pierre.vanmoerbeke@uclouvain.be

vanmoerbeke@brandeis.edu 